\title{
Constraining Unparticles from Top Physics at TeVatron
}

\author{
Mamta Dahiya ${ }^{a}$, Sukanta Dutta $^{a}$, and Rashidul Islam ${ }^{a, \text { }_{*}}$ \\ ${ }^{a} S G T B$ Khalsa College, University of Delhi. Delhi-11000\%. India. and \\ ${ }^{b}$ Department of Physics and Astrophysics, University of Delhi. Delhi-11000\%. India.
}

\begin{abstract}
We study and analyze the recent observations of the top pair production $\sigma(p \bar{p} \rightarrow t \bar{t})$ at TeVatron through flavor conserving and flavor violating channels via vector and tensor unparticles. The unparticle sector is considered with the possibility of being a color singlet or octet. The modified unparticle propagator is used to investigate the contribution of these unparticles to the observed $A_{F B}^{t \bar{t}}$ (forward backward asymmetry in top pair production) and the spin correlation at TeVatron. We have also studied the impact of the flavor violating couplings of unparticles to the third generation quarks on (a) pair production of same sign tops/antitops $\sigma(p \bar{p} \rightarrow t t+\overline{t t})$ at TeVatron and (b) the partial top decay width for $\Gamma_{\mathcal{U}}\left(t \rightarrow u \mathcal{U}^{V}\right)$.

We find that a large region of parameter space is consistent with the measurements of $t \bar{t}$ production cross-section, $A_{F B}^{t \bar{t}}$ and spin correlation coefficient at TeVatron and observe that the top decay width measurement constrains the flavor violating coupling of vector unparticles more severely than the same sign top/antitop production at TeVatron. We also predict the best point-set in the model parameter space for specific choices of $d_{\mathcal{U}}$ corresponding to $\chi_{\min }^{2}$ evaluated using the $m_{t \bar{t}}$ spectrum of $A_{F B}^{t \bar{t}}$ from the data set of Run II of TeVatron at the integrated luminosity $8.7 \mathrm{fb}^{-1}$. Our results and analysis are consistent even with unparticle theories having broken scale invariance as long as the infrared cut-off scale is much less than the top pair production threshold.
\end{abstract}

PACS numbers: 14.80.-j, 12.90.+b, 12.60.-i, 12.38.Qk, 13.66.Hk, 13.90.+i, 14.65.Ha

Keywords: unparticle, top, forward-backward asymmetry, spin correlation, TeVatron

\section{INTRODUCTION}

The study of the top quark production and related discrepancies at TeVatron and LHC might hold key to new physics beyond standard model (SM). Both CDF and $\mathrm{D} \varnothing$ collaborations have consistently measured values of $t \bar{t}$ production cross-sections through various decay channels [1 $\left[\begin{array}{l}3 \\ 3\end{array}\right]$ and they are all consistent with the theoretical predictions at NNLO level [4, 5]. On the other hand the top quark forward-backward asymmetry is observed to be significantly larger than what the SM predicts [6 - 10]. The recent measurements of $A_{F B}^{t \bar{t}}$ at CDF obtains parton level asymmetry to be $0.296 \pm 0.067$ for $m_{t \bar{t}}>450$ $\mathrm{GeV}$ with $8.7 \mathrm{fb}^{-1}$ of data in contrast to the NLO QCD prediction of 0.100 [7]. It has also been observed that in the $t \bar{t}$ rest frame, the asymmetry increases with the $t \bar{t}$ rapidity difference and with the invariant mass. If this asymmetry is true, it should indicate the presence of new physics. Hence the study of this subject has drawn a lot of attention and various explanations have been given for the observed deviations in the context of different new physics scenarios [11 16]. Any model trying to account for the high values of $A_{F B}^{t t}$ is constrained by the SM consistency in the measured cross-section and $m_{t \bar{t}}$ spectrum of $A_{F B}^{t \bar{t}}$.

In the present article we examine the forwardbackward (FB) asymmetry and spin correlations in toppair production at TeVatron with the possibility of existence of a conformally invariant hidden sector containing

*Electronic address: rislam@physics.du.ac.in unparticles coupling weakly with SM fields [17]. The effective couplings of unparticle with SM fields are likely to interfere with the SM processes and hence affect the $A_{F B}^{t \bar{t}}$ and the top spin correlations. Effects of unparticles on top-antitop quark pair production process at hadron colliders and ILC have been studied in references [18 20].

We organize the paper as follows: Calculation of forward-backward asymmetry $A_{F B}^{t \bar{t}}$ and that of spin correlation $C^{t \bar{t}}$ are discussed in sections $\amalg A$ and $\amalg$ respectively. We review the unparticle scenario in section [II] Numerical results for the flavor conserving and violating interactions are given in section [V] Section $\mathrm{V}$ analyses the constraints to the flavor violating channels emerging from two important experimental signatures (a) same sign top production and the (b) top decay width. Section VI presents the $\chi^{2}$ analysis of the model and exhibits the $m_{t \bar{t}}$ distribution of $A_{F B}^{t \bar{t}}$. This section also addresses the implication of the broken scale invariance by introduction of a mass gap and finally summarizes the observations.

\section{THE OBSERVABLES}

\section{A. Forward-Backward Asymmetry}

The $t \bar{t}$ differential charge asymmetry at the partonic level is defined as $A_{C}(\cos \theta)=\frac{N_{t}(\cos \theta)-N_{\bar{t}}(\cos \theta)}{N_{t}(\cos \theta)+N_{\bar{t}}(\cos \theta)}$, where $N_{t}(\cos \theta)=d \sigma^{t \bar{t}} / d(\cos \theta), \theta$ being the polar angle of the top quark momentum with respect to the incoming parton in the $t \bar{t}$ rest frame (which is same as $q \bar{q}$ rest frame for $q \bar{q} \rightarrow t \bar{t}$, the dominant production process at the TeVatron) while in the lab frame it will correspond to the polar angle between the top quark and the proton beam. 
The charge conjugation invariance of the strong interaction would imply $N_{t}(\cos \theta)=N_{\bar{t}}(-\cos \theta)$ and the difference in the production of top quarks in the forward and backward hemispheres is equivalent to the difference in the production of top and antitop quarks in the forward hemisphere. Thus the integrated FB asymmetry in the lab frame is equivalent to the charge asymmetry and can be written as

$$
A_{F B}^{t \bar{t}}=\frac{N_{t}(\cos \theta \geq 0)-N_{t}(\cos \theta \leq 0)}{N_{t}(\cos \theta \geq 0)+N_{t}(\cos \theta \leq 0)}
$$

Collinear initial-state radiation (ISR) makes the fundamental $q \bar{q}$ frame inaccessible in both experiment and simulation, leaving a choice between the $t \bar{t}$ rest frame or the lab $(p \bar{p})$ frame. The direction of top quark in the lab frame can be determined using cosine of the polar angle between the hadronically decaying top quark and the proton beam. However the information on fundamental production asymmetry in the lab frame is diluted because of an uncontrolled longitudinal boost from the rest frame of primary $q \bar{q}$ interaction to the laboratory frame. In the $t \bar{t}$ rest frame, a measurement of the variable $\cos \theta$ in equation (11) requires reconstruction of the initial parton $(q \bar{q})$ rest frame which is not accessible experimentally. Hence instead of $\cos \theta$, the rapidity difference $\Delta y$ between top quark $y_{t}$ and the anti-top quark $y_{\bar{t}}$ is considered as the sensitive variable for the measurement of $A_{F B}^{t \bar{t}}$ experimentally in the $t \bar{t}$ rest frame. This variable being Lorentz invariant can be measured in the lab frame and the shape of rapidity distributions $d \sigma / d y$ in the two frames would remain the same if the boost is by a constant velocity. Experimentally the rapidity difference $\Delta y$ is calculated from the rapidity difference $y_{l_{t}^{+}\left(l_{\bar{t}}^{-}\right)}-y_{h_{\bar{t}}\left(h_{t}\right)}$ of the semileptonically decaying top and the hadronically decaying top [10] and the top rapidity in the $t \bar{t}$ rest frame comes out to be $\frac{1}{2} \Delta y$ in the limit of small $p_{T}$. The variables $\Delta y$ and $\cos \theta$ are directly related by the relation

$$
y_{t}-y_{\bar{t}}=\Delta y=2 \tanh ^{-1}\left(\frac{\cos \theta}{\sqrt{1-\frac{4 m_{t}^{2}}{\hat{s}}}}\right)
$$

$m_{t}$ being the top mass and $\hat{s}$ the square of the center of masss energy of the $t \bar{t}$ pair. Thus $\Delta y$ is a close estimate of the production angle in the $t \bar{t}$ frame. Also the sign of rapidity difference is same as $\cos \theta$ and thus the asymmetry in $\Delta y$ is identical to asymmetry in top production angle $\cos \theta$ in the $t \bar{t}$ rest frame, allowing an effective measurement in the $t \bar{t}$ frame. Hence $\mathrm{D} \varnothing$ and CDF have considered the following observable which also reflects the integrated charge asymmetry in $t \bar{t}$ production at TeVatron:

$$
A_{F B}^{t \bar{t}}=\frac{N[\Delta y \geq 0]-N[\Delta y \leq 0]}{N[\Delta y \geq 0]+N[\Delta y \leq 0]}=\frac{N_{+}-N_{-}}{N_{+}+N_{-}}
$$

$N_{+}\left(N_{-}\right)$being the number of events with a positive (negative) rapidity difference.
Within SM the differential distributions of top and anti-top are identical at leading order in $\alpha_{s}$. At next-toleading order $(\mathrm{NLO})\left(\mathcal{O}\left(\alpha_{s}^{3}\right)\right)$ the dominant positive $\mathrm{FB}$ asymmetry is generated from the interference between the Born amplitude and two-gluon exchange (box) along with a negative asymmetry from the interference of initial state and final state gluon bremsstrahlung (figure 11). The inclusive charge asymmetry receives contribution from the radiative corrections to quark antiquark annihilation (mentioned above) and also from interference between various amplitudes contributing to quark-gluon scattering $(q g \rightarrow t \bar{t} q$ and $\bar{q} g \rightarrow t \bar{t} \bar{q})$, the latter contribution being much smaller than the former. Gluon-gluon fusion remains charge symmetric and $g g$ initial state does not contribute to this asymmetry at any order in perturbation theory, due to the fact that the gluon distribution is the same for protons and antiprotons. It however lowers the average value. QCD predicts the size of this asymmetry to be 5 to $8 \%$ 21, 22]. The measurement of the total FB asymmetry has been carried out by CDF and D $\varnothing$ collaborations at TeVatron in both laboratory frame ( $p \bar{p}$ frame) as well as the center-of-mass frame of the top pair ( $t \bar{t}$ frame) [6, 9, 10] (see table \). While the other TeVatron measurements of top quark properties (e.g. production cross-section) are all consistent with the SM, all the past measurements of CDF and $\mathrm{D} \varnothing$ have consistently yielded a higher $A_{F B}^{t \bar{t}}$ than SM prediction by more than $2 \sigma$ deviation (see table $\llbracket$ ). CDF has also

\begin{tabular}{|c|c|}
\hline \hline Description & $\begin{array}{c}\text { Value of } A_{F B}^{t t} \text { in specific frame } \\
(p \bar{p} \text { rest frame or } t \bar{t} \text { rest frame })\end{array}$ \\
\hline $\begin{array}{c}\text { CDF }\left(L=8.7 \mathrm{fb}^{-1}\right) \\
(\text { semileptonic })\end{array}$ & $0.162 \pm .047^{\text {stat }+ \text { syst }}(t \bar{t})[7]$ \\
\hline $\begin{array}{c}\mathrm{CDF}\left(L \sim 5 \mathrm{fb}^{-1}\right) \\
(\text { combined })\end{array}$ & $0.201 \pm .067^{{ }^{\text {stat }+ \text { syst }]}[8]}$ \\
\hline $\begin{array}{c}\mathrm{CDF}\left(L=5.1 \mathrm{fb}^{-1}\right) \\
(\text { dileptonic })\end{array}$ & $0.42 \pm .15^{\text {stat }} \pm .05^{\text {(syst) }}(t \bar{t})[9]$ \\
\hline $\begin{array}{c}\mathrm{D} \varnothing\left(L=5.4 \mathrm{fb}^{-1}\right) \\
(\text { lepton+jets })\end{array}$ & $(15.2 \pm 4.0) \%(t \bar{t})[6]$ \\
\hline \hline SM (NNLO) & $0.052_{-0.006}^{+0.000}(p \bar{p})[22]$ \\
\hline \hline
\end{tabular}

TABLE I: Values of Forward-Backward Asymmetry as measured at CDF and DØ along with SM theoretical Value.

released the functional dependence of the $t \bar{t}$ rest frame asymmetry on $\Delta y$ and on $m_{t \bar{t}}$ [7].

There are many efforts [11] in the literature to explain the large excess of top asymmetry observed at TeVatron. A challenge is to realize a model which can generate a large $A_{F B}^{t \bar{t}}$ without making an appreciable change in the observed $t \bar{t}$ production cross section or invariant mass spectrum. The all channel measurement from CDF with $4.6 \mathrm{fb}^{-1}$ of data [1] is $\sigma(t \bar{t})=7.5 \pm 0.31$ (stat) \pm 0.34 (syst) \pm 0.15 (Z theory) pb for $m_{t}=172.5 \mathrm{GeV}$, in good agreement with the SM prediction of $\sigma(t \bar{t})_{S M}^{N N L O}=$ $7.08_{-0.24-0.27}^{+0.00+0.36} \mathrm{pb}$ for $m_{t}=173 \mathrm{GeV}$ [4] and is consistent 

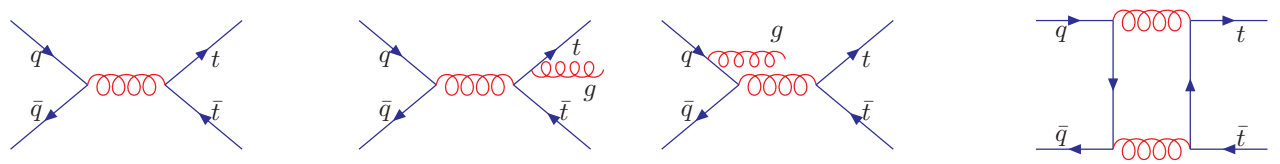

FIG. 1: QCD diagrams contributing to charge asymmetry in top pair production.

with measurements from $\mathrm{D} \varnothing[\underline{3}]$.

References [13] and 14] have compared various models in light of CDF measurement of $A_{F B}^{t \bar{t}}$ and invariant mass distributions. They show that axigluon and heavy $Z^{\prime}$ models are highly constrained while reference [15] further demonstrate that $Z^{\prime}$ and $W^{\prime}$ models are disfavored by the LHC measurements.

Reference [16] studies the $A_{F B}^{t \bar{t}}$ in the framework of unparticles. They have only considered a colored octet vector unparticle in the s-channel having flavor conserving couplings to quarks. We shall compare our results for this particular case with them.

\section{B. Spin Correlation}

Due to its large mass the top quark decays before hadronization leading to preservation of its spin information. In the leading order in SM, top quarks remain unpolarized because they are mainly produced by parity conserving QCD interaction. The decay of top quarks via electroweak interaction which is negligible with respect to the strong interaction leaves very small effect on top polarization 23]. The angular distribution of the partial top quark decay width $\Gamma$ in $t \rightarrow W^{+}+b$ followed by $W^{+} \rightarrow l^{+}+\nu$ or $\bar{d}+u$ are correlated with the top spin axis [24, 25] as follows:

$$
\frac{1}{\Gamma_{t}} \frac{d \Gamma}{d \cos \chi_{i}}=\frac{1}{2}\left(1+\alpha_{i} \cos \chi_{i}\right) ; \alpha_{i}=\left\{\begin{array}{l}
+1 \text { for } l^{+}, \bar{d} \\
-.31 \text { for } \bar{\nu}, u \\
-.41 \text { for } b
\end{array}\right.
$$

where $\Gamma_{t}$ is the total top decay width and $\chi_{i}$ is the angle between the $i$-th decay product and the top quark spin axis in the top quark rest frame. Since net polarization of the top quarks is negligible in leading order, the correlation between the $i$-th decay product of the top and $\bar{\imath}$-th decay product of the antitop can be expressed by

$$
\frac{1}{\sigma_{t \bar{t}}} \frac{d^{2} \sigma_{i \bar{i}}}{d \cos \chi_{i} d \cos \bar{\chi}_{\bar{i}}}=\frac{1}{4}\left(1+C^{t \bar{t}} \alpha_{i} \bar{\alpha}_{\bar{i}} \cos \chi_{i} \cos \bar{\chi}_{\bar{i}}\right)
$$

with

$$
C^{t \bar{t}}=\frac{\sigma_{\uparrow \uparrow}+\sigma_{\downarrow \downarrow}-\sigma_{\uparrow \downarrow}-\sigma_{\downarrow \uparrow}}{\sigma_{\uparrow \uparrow}+\sigma_{\downarrow \downarrow}+\sigma_{\uparrow \downarrow}+\sigma_{\downarrow \uparrow}} .
$$

$\sigma_{\uparrow / \downarrow \uparrow / \downarrow}$ is the production cross section for top quark pairs where the top quark has spin up or down with re- spect to the top spin axis and the antitop has spin up or down with respect to the antitop spin axis.

Mahlon et al discussed the right choice for the spin axes of the top quark pair since a poor choice of spin axes can lead to a small value of $C^{t \bar{t}}[26]$. They proposed three choices for the spin axis, namely helicity basis, beamline basis and Off-diagonal basis.

We choose the helicity basis (top quark momentum is chosen as spin quantization axis) for our calculation. In $t \bar{t}$ rest frame the quarks move back-to-back and the same spin $(S=1)$ states are those with opposite helicity so that in helicity basis

$$
C^{t \bar{t}}=\frac{\sigma_{R L}+\sigma_{L R}-\sigma_{R R}-\sigma_{L L}}{\sigma_{R R}+\sigma_{L L}+\sigma_{R L}+\sigma_{L R}}
$$

The dominant production mechanism for the $t \bar{t}$ pairs at the TeVatron is $q q \rightarrow t \bar{t}$ with a $J=1$ gluon exchanged in $s$-channel. Near threshold, the $t \bar{t}$ pair is produced in $S=1$ state with the eigenstates $\mid++>, \frac{1}{\sqrt{2}}(\mid+->$ $+\mid-+>), \mid-->$. Two of the three states have the opposite helicity (same spin), hence the correlation near threshold is $C_{t \bar{t}}=33 \%$ while helicity conservation at high energy ensures that the $t$ and $\bar{t}$ are produced with the opposite helicity and $C_{t \bar{t}}=100 \%$ at very high energies 27]. CDF has reported the value of top spin correlation in the helicity basis to be $0.60 \pm 0.50$ (stat) \pm 0.16 (syst) [28] and in the beam basis to be $0.042_{-.562}^{+0.563}$ [29]. The $\mathrm{D} \emptyset$ measurement for spin correlation coefficient is $-.66<$ $-C^{t \bar{t}}<0.81$ in the beam basis [30]. The SM values for the same in beam and helicity basis are -0.614 and 0.299 respectively [31].

The terms linear in $\cos \theta$ do not contribute to $C^{t \bar{t}}$ after integration over $\cos \theta$. Thus $C^{t \bar{t}}$ and $A_{F B}^{t \bar{t}}$ are sensitive to different terms. To relate the dependence of the two observables on the chiral structure of the quark couplings to any new physics sector, recently Ko et. al. proposed a new spin-spin FB asymmetry [12] which is defined as

$$
\begin{aligned}
C_{F B}^{t \bar{t}} & \equiv C^{t \bar{t}}(\cos \theta \geq 0)-C^{t \bar{t}}(\cos \theta \leq 0) \\
& =A_{F B}^{t \bar{t}}(\text { opp hel })-A_{F B}^{t \bar{t}}(\text { same hel })
\end{aligned}
$$

while the charge asymmetry may be written as

$$
A_{F B}^{t \bar{t}}=A_{F B}^{t \bar{t}}(\text { opp hel })+A_{F B}^{t \bar{t}}(\text { same hel })
$$

Since there is no contribution to $A_{F_{B}}^{t \bar{t}}$ in SM from same helicity states, for SM $C_{F B}^{t \bar{t}}=A_{F B}^{t \bar{t}}($ opp hel $)=A_{F B}^{t \bar{t}}$. 
The correlation between $C_{F B}^{t \bar{t}}$ and $A_{F B}^{t \bar{t}}$ can help to distinguish between various new physics scenarios.

New physics can show up both at the production of top quarks as well as decay. If the new physics contains chiral asymmetry then it will affect the top spin correlation appreciably and also the forward-backward spin correlation asymmetry [20, 32, 33].

\section{THE MODEL}

It is known that the visible particle sector is based on theories that are free in infrared and/or have a mass gap. In contrast, an exact conformal invariance would require the mass spectrum to be either continuous or all zero masses. Although there exist interacting conformal theories that have an infra red fixed point but such theories do not have asymptotically free in and out states and the traditional $S$-matrix description does not work. The theory of unparticles as an conformally invariant sector that is weakly coupled to the SM particles was proposed by Georgi [17] which was motivated by Banks-Zaks theory [34]. This assumes the existence of a hidden sector with non-trivial infrared fixed point (e.g. Banks-Zaks type) that interacts with the SM through the exchange of messenger field with a large mass $M$. Below the scale $M$, one can integrate out the heavy field giving rise to the effective non-renormalizable couplings of the form

$$
\frac{C_{i}}{M^{d_{\mathrm{UV}}+d_{\mathrm{SM}}^{i}-4}} \mathcal{O}_{\mathrm{SM}}^{i} \mathcal{O}_{\mathrm{UV}}
$$

where $C_{i}$ are the dimensionless coupling constants, $\mathcal{O}_{\mathrm{SM}}^{i}$ and $\mathcal{O}_{\mathrm{UV}}$ are respectively the local operators built out of SM fields and hidden sector fields having scaling dimensions $d_{\mathrm{SM}}^{i}$ and $d_{\mathrm{UV}}$ respectively. The hidden sector has an infrared fixed point and becomes conformal at some scale say $\sim \Lambda<M$. Below the energy scale $\Lambda$, the renormalizable couplings of the hidden sector fields cause dimensional transmutation. In the effective theory the high energy operators $\mathcal{O}_{\text {UV }}$ above this scale match onto the unparticle operators $\mathcal{O}_{\mathcal{U}}$ (the operator $\mathcal{O}_{\text {UV }}$ becomes $\Lambda^{d_{\mathrm{UV}}-d_{\mathcal{U}}} \mathcal{O}_{\mathcal{U}}$ ) below this scale and the interactions of equation (11) now take the form

$$
\frac{C_{i} \Lambda^{d_{\mathrm{UV}}-d_{\mathcal{U}}}}{M^{d_{\mathrm{UV}}+d_{\mathrm{SM}}^{i}-4}} \mathcal{O}_{\mathrm{SM}}^{i} \mathcal{O}_{\mathcal{U}}=\frac{C_{i}}{\Lambda_{\mathcal{U}}^{d_{\mathrm{SM}}^{i}+d_{\mathcal{U}}-4}} \mathcal{O}_{\mathrm{SM}}^{i} \mathcal{O}_{\mathcal{U}}
$$

where $d_{\mathcal{U}}$ is the scaling dimension of the operator $\mathcal{O}_{\mathcal{U}}$. $M, \Lambda, d_{\mathcal{U}}$ and $d_{\mathrm{UV}}$ are the hidden sector parameters while the exponent of $\Lambda_{\mathcal{U}}$ depends upon the dimension of the SM operator and is given by

$$
\Lambda_{\mathcal{U}}^{d_{\mathrm{SM}}^{i}+d_{\mathcal{U}}-4}=\frac{M^{d_{\mathrm{UV}}+d_{\mathrm{SM}}^{i}-4}}{\Lambda^{d_{\mathrm{UV}}-d_{\mathcal{U}}}}
$$

The operators with different mass dimension are likely to couple with different strengths. The couplings $C_{i}$ and the scale $\Lambda$ only appear in a combination given by (12) and there is no guiding theoretical principle to fix $C_{i}$. In addition to the interaction of SM fields with unparticle which can be probed at high energy colliders (below the scale $\Lambda$ ), the elimination of heavy fields also induces the contact interaction among SM fields which are suppressed by powers of $M$ and have the effect of drowning down any unparticle effects for $d_{\mathcal{U}} \gg 1$.

Unparticle effects are detected in the colliders either through missing energy distributions or by the interference effects with SM amplitudes. But the constraints from astrophysics and cosmology [35] would render them practically undetectable in the collider experiments unless otherwise they break the scale invariance at $\gtrsim 1 \mathrm{GeV}$. The conformal invariance may be broken at a scale $\mu$ by the higgs-unparticle coupling $H^{\dagger} H \mathcal{O}_{\mathcal{U}}$ which introduces a scale in the theory once higgs acquires vacuum expectation value. For consistency $\mu \leq \Lambda$ and the two scales should be well separated to give a window where the sector is conformal. Thus for scales $\Lambda$ and $M$ to be experimentally accessible, the higgs-scalar unparticle coupling is assumed to break scale invariance at electroweak scale. However, if only vector unparticles are present, scale invariance is broken by higher dimensional operators leading to the breaking of the scale invariance below the electroweak scale [36].

Initially we have not introduced the effect of the breaking scale invariance with respect to the study of the top pair production and the same sign top production at TeVatron. Nonetheless we have shown its effect in the evaluation of the top decay width. Introduction of such modifications in the theory do not change the cross sections appreciably if $\mu \ll 2 m_{t}$. The phenomenological lower bound on the scale invariance breaking scale comes from the BBN and SN 1987A where $\mu$ is required to be sufficiently large compared to the relevant energy scales $\simeq 1 \mathrm{MeV}$ and $\simeq 30 \mathrm{MeV}$ respectively [36]. We dwell on these issues in the appropriate sections and also in the subsection VIB.

The two-point function of unparticle operators [37] is written as

$$
\left\langle 0\left|\mathcal{O}_{\mathcal{U}}(x) \mathcal{O}_{\mathcal{U}}^{\dagger}(0)\right| 0\right\rangle=\int \frac{d^{4} p}{(2 \pi)^{2}} e^{-i p \cdot x} \rho\left(p^{2}\right),
$$

where $\rho\left(p^{2}\right)=(2 \pi)^{2} \int d \lambda \delta^{4}\left(p-p_{\lambda}\right)\left|\left\langle 0\left|\mathcal{O}_{\mathcal{U}}\right| \lambda\right\rangle\right|^{2}$. The spectral function $\rho\left(p^{2}\right)$ is determined by scale invariance to be $\rho\left(p^{2}\right)=A_{d_{\mathcal{U}}} \theta\left(p^{0}\right) \theta\left(p^{2}\right)\left(p^{2}\right)^{d_{\mathcal{U}}-2}$, where $A_{d_{\mathcal{U}}}$ is the normalization factor. This factor is fixed by identifying $\rho\left(p^{2}\right)$ with $d_{\mathcal{U}}$-body phase space of massless particles to be

$$
A_{d_{\mathcal{U}}}=\frac{16 \pi^{2} \sqrt{\pi}}{(2 \pi)^{2 d_{\mathcal{U}}}} \frac{\Gamma\left(d_{\mathcal{U}}+1 / 2\right)}{\Gamma\left(d_{\mathcal{U}}-1\right) \Gamma\left(2 d_{\mathcal{U}}\right)} .
$$

The scaling dimension $d_{\mathcal{U}}$ can have non-integral values as well. With the use of the spectral function $\rho\left(p^{2}\right)$ and requiring scale invariance, the Feynman propagators for 


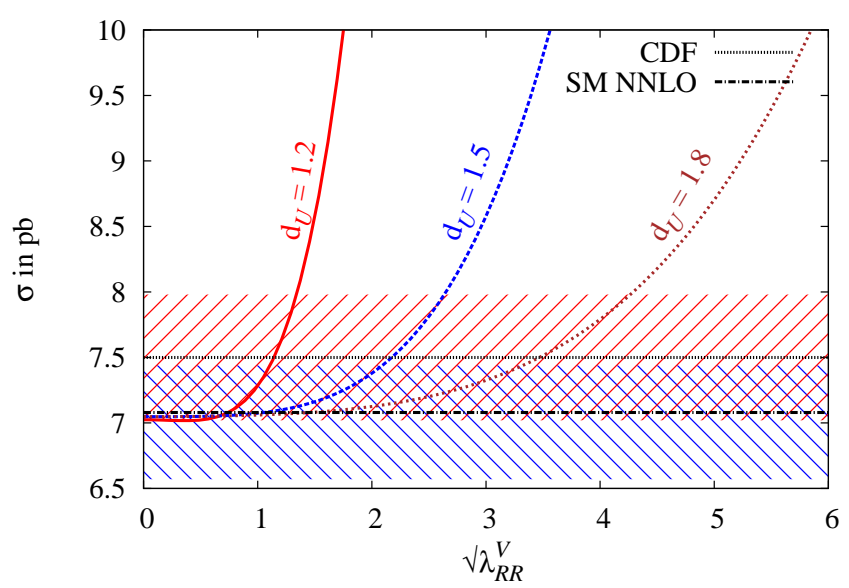

(a) $\lambda_{R R}^{V} \neq 0$ and $\lambda_{L R}^{V}=\lambda_{R L}^{V}=\lambda_{L L}^{V}=0$

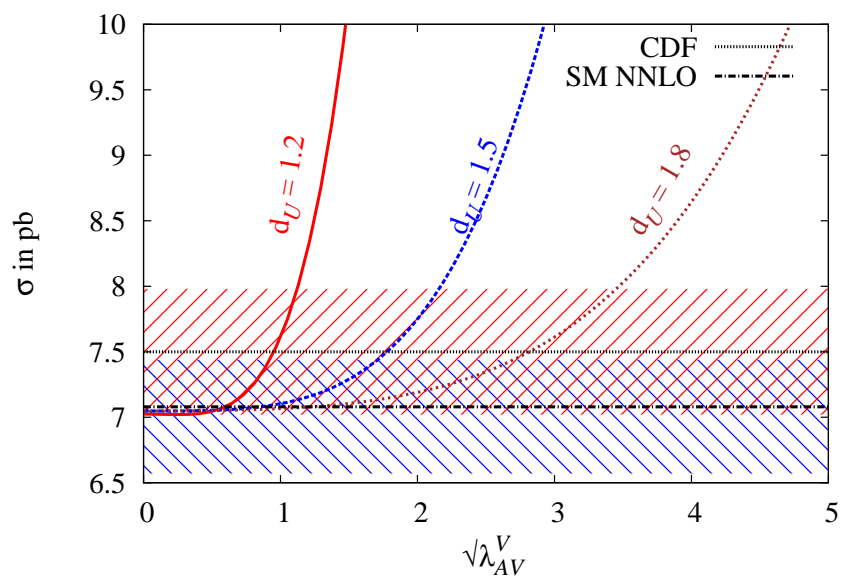

(c) $\lambda_{L L}^{V}=\lambda_{R R}^{V}=-\lambda_{R L}^{V}=-\lambda_{L R}^{V}=\lambda_{A V}^{V}$

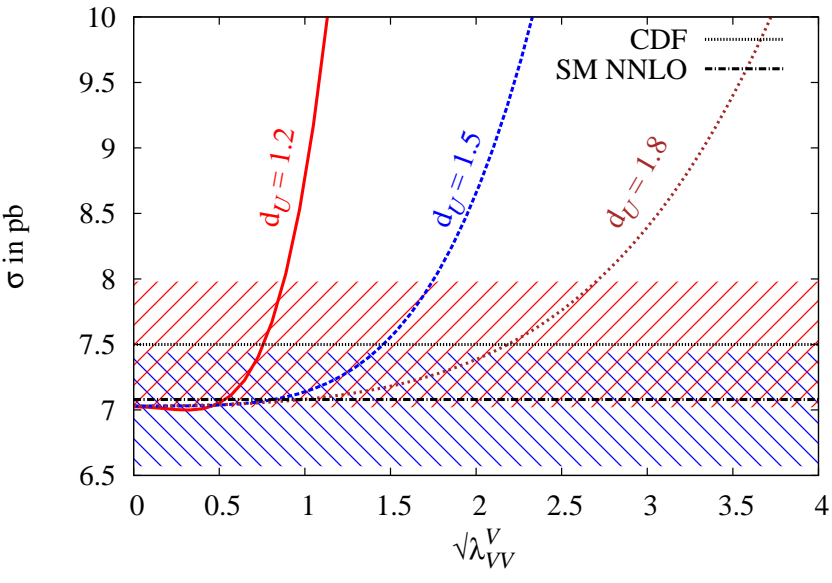

(b) $\lambda_{L L}^{V}=\lambda_{R R}^{V}=\lambda_{R L}^{V}=\lambda_{L R}^{V}=\lambda_{V V}^{V}$

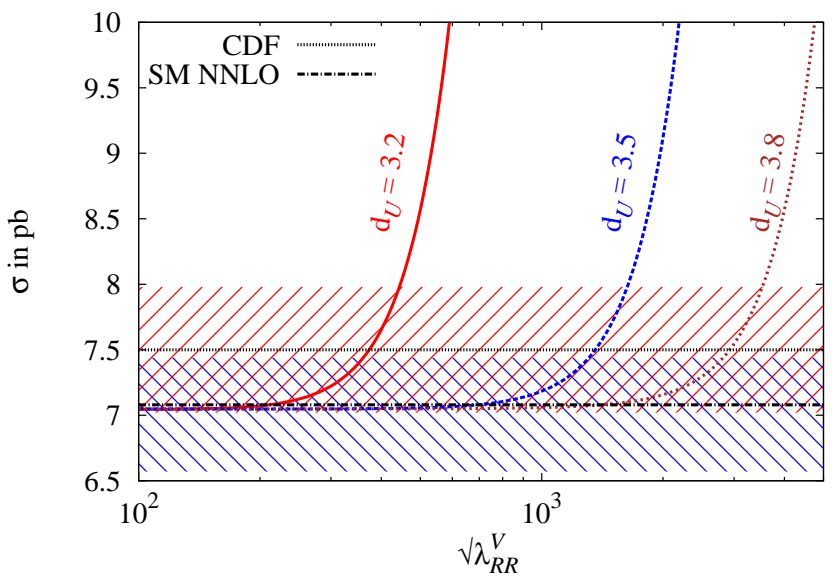

(d) $\lambda_{R R}^{V} \neq 0$ and $\lambda_{L R}^{V}=\lambda_{R L}^{V}=\lambda_{L L}^{V}=0$

FIG. 2: Variation of the cross-section $\sigma(p \bar{p} \rightarrow t \bar{t})$ with couplings $\sqrt{ } \lambda_{i j}^{V}$ for color singlet flavor conserving vector unparticles corresponding to different values of $d_{\mathcal{U}}$ at fixed $\Lambda_{\mathcal{U}}=1 \mathrm{TeV}$. The upper dotted line with a red band depicts the cross-section $7.50 \pm 0.48 \mathrm{pb}$ from CDF (all channels) [1], while the lower dot-dashed line with a blue band show theoretical estimate $7.08 \pm$ $0.36 \mathrm{pb}$ at NNLO [4]. The panels ' $a$ ', ' $b$ ' and ' $c$ ' show the variation of $\sigma$ for different combinations of couplings (the cases (a) (or b), (c) and (d) of the text respectively) and for $d_{\mathcal{U}}$ in the range $1<d_{\mathcal{U}}<2$ while the panel 'd' corresponds to the variation in the $d_{\mathcal{U}}$ range $3<d_{\mathcal{U}}<4$ for the coupling combination (a) mentioned in the text.

vector and tensor unparticle are defined to be [37]

where

$$
\begin{aligned}
\Delta_{\mu \nu}\left(p^{2}\right) & =\frac{i A_{d_{\mathcal{U}}}}{2 \sin \left(d_{\mathcal{U}} \pi\right)}\left(-p^{2}\right)^{d_{\mathcal{U}}-2} \Pi_{\mu \nu}(p), \\
\Delta_{\mu \nu, \rho \sigma}\left(p^{2}\right) & =\frac{i A_{d_{\mathcal{U}}}}{2 \sin \left(d_{\mathcal{U}} \pi\right)}\left(-p^{2}\right)^{d_{\mathcal{U}}-2} \mathcal{T}_{\mu \nu, \rho \sigma}(p)
\end{aligned}
$$

$$
\Pi^{\mu \nu}(p)=-g^{\mu \nu}+\frac{p^{\mu} p^{\nu}}{p^{2}}, \quad \mathcal{T}^{\mu \nu, \rho \sigma}(p)=\frac{1}{2}\left\{\Pi^{\mu \nu}(p) \Pi^{\nu \sigma}(p)+\Pi^{\mu \sigma}(p) \Pi^{\nu \rho}(p)-\frac{2}{3} \Pi^{\mu \nu}(p) \Pi^{\rho \sigma}(p)\right\}
$$

and $\left(-p^{2}\right)^{d_{\mathcal{U}}-2}$ is interpreted as

Propagators are chosen such that they satisfy the rela-

$$
\left(-p^{2}\right)^{d_{\mathcal{U}}-2}= \begin{cases}\left|p^{2}\right|^{d_{\mathcal{U}}-2} & \text { for } p^{2}<0 \\ \left|p^{2}\right|^{d_{\mathcal{U}}-2} e^{i d_{\mathcal{U}} \pi} & \text { for } p^{2}>0\end{cases}
$$


tions $p_{\mu} \Pi^{\mu \nu}(p)=0, p_{\mu} \mathcal{T}^{\mu \nu, \rho \sigma}(p)=0$, and $\mathcal{T}_{\mu}^{\mu, \rho \sigma}(p)=0$. Also the unparticle operators are all taken to be hermitian, $\mathcal{O}_{\mathcal{U}}{ }^{\mu}$ and $\mathcal{O}_{\mathcal{U}}{ }^{\mu \nu}$ are assumed to be transverse and the spin-2 unparticle operator is taken to be traceless $\mathcal{O}_{\mathcal{U}}^{\mu}=0$

The negative sign in front of $p^{2}$ of the second term gives rise to a unique phase factor for time like virtual unparticles but not for the space like. This leads to interesting interference effects with SM process which will be discussed later.

Based on this template of the unparticle formalism, possible signatures of scalar, vector and tensor unparticles at colliders and their effects on low energy phenomenology have been studied [18, 19, 38]. Astrophysics and cosmology also put strong constraints on unparticles [35, 39]. Various theoretical aspects of unparticles have been also studied [40, 41].

Grinstein et al [41] have revisited the computation of two point function for unparticles demanding rigid conformal invariance in the hidden sector and shown that the unitarity of conformal algebra imposes lower bounds on

scaling dimensions of the vector and tensor operators [41]. Thus $d_{\mathcal{U}}{ }^{V} \geq 3$ and $d_{\mathcal{U}}{ }^{T} \geq 4$ for vector unparticle and symmetric traceless tensor unparticle respectively. The primary vector operator $\mathcal{O}_{\mathcal{U}}{ }^{\mu}$ with $\partial_{\mu} \mathcal{O}_{\mathcal{U}}{ }^{\mu}=0$ corresponds to particle with $d u^{V}=3$, while the vector unparticle corresponding to $\partial_{\mu} \mathcal{O}_{\mathcal{U}}{ }^{\mu} \neq 0$ and $d_{u}>3$ get a modified propagator

$$
\Delta^{\mu \nu}(p)=\frac{-i A_{d_{\mathcal{U}}}}{2 \sin \left(d_{\mathcal{U}} \pi\right)}\left(-p^{2}\right)^{d_{\mathcal{U}}-3}\left(p^{2} g^{\mu \nu}-a p^{\mu} p^{\nu}\right),
$$

with $a=2\left(d_{\mathcal{U}}-2\right) /\left(d_{\mathcal{U}}-1\right)$. It is to be noted that this differs from the propagator given in equation (16) not only in the relative size of the terms but also by an overall extra phase factor $e^{-i \pi}$. This extra phase would affect the sign of interference term with SM processes. In fact this contradicts the many observations made in the literature with respect to the unparticles. Similarly the tensor unparticle propagators is modified to [42]

$$
\Delta_{\mu \nu, \alpha \beta}\left(p^{2}\right)=\frac{A_{d_{\mathcal{U}}}}{2 \sin \left(d_{\mathcal{U}} \pi\right)}\left(-p^{2}\right)^{d_{\mathcal{U}}-2} \mathcal{T}_{\mu \nu, \alpha \beta},
$$

where

$$
\begin{aligned}
\mathcal{T}_{\mu \nu, \alpha \beta}(p)= & d_{\mathcal{U}}\left(d_{\mathcal{U}}-1\right)\left(g_{\mu \alpha} g_{\nu \beta}+\mu \leftrightarrow \nu\right)+\left[2-\frac{d_{\mathcal{U}}}{2}\left(d_{\mathcal{U}}+1\right)\right] g_{\mu \nu} g_{\alpha \beta} \\
& -2\left(d_{\mathcal{U}}-1\right)\left(d_{\mathcal{U}}-2\right)\left(g_{\mu \alpha} \frac{p_{\nu} p_{\beta}}{p^{2}}+g_{\mu \beta} \frac{p_{\nu} p_{\alpha}}{p^{2}}+\mu \leftrightarrow \nu\right) \\
& +4\left(d_{\mathcal{U}}-2\right)\left(g_{\mu \nu} \frac{p_{\alpha} p_{\beta}}{p^{2}}+g_{\alpha \beta} \frac{p_{\mu} p_{\nu}}{p^{2}}\right)+8\left(d_{\mathcal{U}}-2\right)\left(d_{\mathcal{U}}-3\right) \frac{p_{\mu} p_{\nu} p_{\alpha} p_{\beta}}{\left(p^{2}\right)^{2}} .
\end{aligned}
$$

We have considered the symmetric structure for the tensor-propagator.

In this article we explore the phenomenologically viable and interesting scale invariant (not strictly conformally invariant) hidden sector where the bounds on $d_{\mathcal{U}}{ }^{V}, d_{\mathcal{U}}{ }^{T}$ are relaxed. We do investigate the sensitivity of the observables with the full conformal invariance also though it should be kept in mind that for such large values of $d_{\mathcal{U}}$, the SM contact interactions (induced at scale $M$ by the exchange of ultra heavy particle) dominate over the unparticle-SM interference effects.

The relevant unparticle-SM flavor conserving (FC) and flavor violating (FV) interaction Lagrangian is given in equations A.3 A.6 at the Appendix. We consider the vector and tensor unparticles with the possibility of each being a singlet or octet under $S U(3)_{C}$. Since gluons interact with vector unparticles through a derivative term $G_{\mu} \alpha G_{\nu}^{\alpha} \partial^{\mu} \mathcal{O}_{\mathcal{U}}^{\nu}$ and not with the primary field $\mathcal{O}_{\mathcal{U}}^{\nu}$, it is suppressed by a factor of $\Lambda_{\mathcal{U}}$ compared to the interaction of vector unparticle with quarks. Moreover, the gluon flux at TeVatron is low and so it is further subdued. Hence we do not consider such interaction terms.

We compute the helicity amplitudes with appropriate color factors corresponding to Lagrangian and present them in the appendix A. In calculating the amplitudes for unparticle we have used the improved propagator of equation (19) for vector and of equation (20) for the tensor unparticle.

The phenomenological consequences of the color octet unparticles are addressed and validated in section VIB. There have been various attempts to provide a complete gauge theory of unparticles [43, 44], however in the absence of any well established approach to a full theory of unparticle interactions we study the topic from a phenomenological point of view.

\section{SENSITIVITY OF THE MODEL PARAMETERS}

We perform a parton level and leading order calculation, where we have used CTEQ6L LHApdf parton distribution function, the top mass $m_{t}=173 \mathrm{GeV} / c^{2}$ and $\alpha_{s}=\alpha_{s}\left(m_{Z}\right)=0.13$. We evolve $\alpha_{s}$ to get $\alpha_{s}\left(2 m_{t}\right)$ using CTEQ6L LHApdf. The tree-level SM cross-section was obtained to be $\sigma_{\mathrm{SM}}^{\text {tree }}=4.26 \mathrm{pb}$, which is consistent with the results available from Herwig ++ [45], CalcHEP [46] and MadGraph/Madevent [47] for the given choice of parameters. We have also matched our results with that existing literature [16, 20]. 
We normalize our tree level SM cross-section to $7.08 \mathrm{pb}$ to include the NNLO corrected matrix element squared [4] with $m_{t}=173 \mathrm{GeV} / c^{2}$. Accordingly we normalise the SM amplitude by a factor $k=\sqrt{7.08 / 4.26}=\sqrt{1.663}$. The $k$ factor is sensitive to the choice of renormalisation and factorization scale but eventually it stabilses with the inclusion of higher order corrections of the matrix element at the NNLO level [5]. As the NLO corrections in the new physics sector is not feasible within the scope of our article, we work with the LO contribution only in the unparticle physics sector. Thus after scaling we may write the squared matrix element as

$$
\begin{aligned}
\left|\mathcal{M}^{t \bar{t}}\right|^{2}=\left|k \mathcal{M}_{\text {tree }}^{\mathrm{SM}}\right|^{2} & +2\left(\mathcal{R} e\left(k \mathcal{M}_{\text {tree }}^{\mathrm{SM}}\right)^{\dagger}\left(\mathcal{M}^{\mathrm{unp}}\right)\right) \\
& +\left|\mathcal{M}^{\mathrm{unp}}\right|^{2}
\end{aligned}
$$

As mentioned earlier SM inclusive processes can generate $A_{F B}^{t \bar{t}}$ which is at most $5-8 \%$ through the radiative diagrams at NLO level, it is worth to examine the additional contribution to the integrated and differential $A_{F B}^{t \bar{t}}$ generated by the LO top pair production processes mediated through unparticles. The contribution of unparticles to cross-section, charge asymmetry and spin correlation coefficient come from the matrix element square term containing only unparticles and the interference of unparticles with SM QCD and electroweak matrix elements. To see the correlation between $C_{F B}^{t \bar{t}}$ and $A_{F B}^{t \bar{t}}$ and dependence on the chiral structure of the theory, we divide these two into same helicity and opposite helicity contributions as

$$
\begin{aligned}
\left|\mathcal{M}^{\text {unp }}\right|^{2}= & \left|\mathcal{M}^{\text {unp }}\right|_{\text {same hel }}^{2}+\left|\mathcal{M}^{\text {unp }}\right|_{\text {opp hel }}^{2} \\
2 \mathcal{R} e\left[\left(\mathcal{M}_{\text {tree }}^{\mathrm{SM}}\right)^{\dagger} \mathcal{M}^{\text {unp }}\right]= & \left.2 \mathcal{R} e\left[\left(\mathcal{M}_{\text {tree }}^{\mathrm{SM}}\right)^{\dagger} \mathcal{M}^{\text {unp }}\right]\right|_{\text {same hel }} \\
& +\left.2 \mathcal{R} e\left[\left(\mathcal{M}_{\text {tree }}^{\mathrm{SM}}\right)^{\dagger} \mathcal{M}^{\text {unp }}\right]\right|_{\text {opp hel }}
\end{aligned}
$$

The helicity amplitudes (as given in the appendix) involve the product of the couplings $g_{i}^{\mathcal{U}_{(V / T)}^{\mathrm{n}} \bar{q} q} g_{j}^{\mathcal{U}_{(V / T)}^{\mathrm{n}}}{ }^{\bar{t} t}=$ $\lambda_{i j}^{(V / T)}$ (with $i$ and $j \equiv L$ or $R$ ) for flavor conserving (FC) vector/tensor unparticles and $g_{i}^{u t}$ for flavor violating $(\mathrm{FV})$ vector unparticles. Therefore the observables are analyzed with the independent free parameters : the scaling dimension $d_{\mathcal{U}}$ and $\lambda_{i j}^{(V / T)}\left(g_{i}^{u t}\right)$ for FC vector/tensor (FV vector) unparticles. Throughout our analysis we have fixed $\Lambda_{\mathcal{U}}=1 \mathrm{TeV} \gg m_{t \bar{t}}$. In all the figures showing the cross-section, we plot the central values with the respective error band for CDF $\sigma^{t \bar{t}}=7.5 \pm 0.48 \mathrm{pb}$ [1] and the SM NNLO 7.08+0.36-0.51 pb [4]. We bound the total contribution from SM and unparticles to the $\sigma^{t \bar{t}}$ for a given $d_{\mathcal{U}}$ at fixed $\Lambda_{\mathcal{U}}=1 \mathrm{TeV}$ within the error bars of CDF data, which in turn gives the upper cut-off for the couplings.

\section{A. Flavor Conserving Unparticle}

The FC couplings for the light quarks are tightly constrained from the measurement of dijet events at TeVatron [48]. Therefore the FC couplings of the light quarks $g_{(L / R)}^{\mathcal{U}_{(V / T)}^{\mathrm{n}} \bar{q} q}$ with the vector and tensor unparticles are taken to be order of magnitude smaller than those of third generation quarks or antiquarks $g_{(L / R)}^{\mathcal{U}_{(V / T)}^{\mathrm{n}} \bar{t} t}$. A detailed discussion on this can be found in the reference [49] for $d_{\mathcal{U}} \geq 3$. We have also verified the dijet spectrum corresponding to $1 \leq d_{\mathcal{U}} \leq 3$ for most of the parameter region probed and it is consistent with the observed data. For FC processes, the products of couplings $\lambda_{i j}^{(V / T)}$ can be broadly classified into four combinations for a given $d_{\mathcal{U}}$ and $\Lambda$.

$$
\begin{aligned}
& \text { (a) } g_{L}^{\mathcal{U}_{(V / T)}^{\mathrm{n}} \bar{q} q}=g_{L}^{\mathcal{U}_{(V / T)}^{\mathrm{n}} \bar{t} t}=0 \text { i.e } \lambda_{L R}^{(V / T)}=\lambda_{R L}^{(V / T)}= \\
& \lambda_{L L}^{(V / T)}=0 \text { and } \lambda_{R R}^{(V / T)} \neq 0 \\
& \text { (b) } g_{R}^{\mathcal{U}_{(V / T)}^{\mathrm{n}} \bar{q} q}=g_{R}^{\mathcal{U}_{(V / T)}^{\mathrm{n}} \bar{t} t}=0 \text { i.e } \lambda_{L R}^{(V / T)}=\lambda_{R L}^{(V / T)}= \\
& \lambda_{R R}^{(V / T)}=0 \text { and } \lambda_{L L}^{(V / T)} \neq 0 \\
& \text { (c) } g_{L}^{\mathcal{U}_{(V / T)}^{\mathrm{n}} \bar{q} q}=g_{R}^{\mathcal{U}_{(V / T)}^{\mathrm{n}} \bar{q} q}, g_{L}^{\mathcal{U}_{(V / T)}^{\mathrm{n}} \bar{t} t}=g_{R}^{\mathcal{U}_{(V / T)}^{\mathrm{n}} \bar{t} t} \text { i.e. } \\
& \lambda_{L L}^{(V / T)}=\lambda_{R R}^{(V / T)}=\lambda_{R L}^{(V / T)}=\lambda_{L R}^{(V / T)}=\lambda_{V V / T T} \\
& \text { (d) } g_{L}^{\mathcal{U}_{(V / T)}^{\mathrm{n}} \bar{q} q}=-g_{R}^{\mathcal{U}_{(V / T)}^{\mathrm{n}}} \bar{q} q, g_{L}^{\mathcal{U}_{(V / T)}^{\mathrm{n}}}{ }^{\bar{t} t}=-g_{R}^{\mathcal{U}_{(V / T)}^{\mathrm{n}}}{ }^{\bar{t} t} \text { i.e. } \\
& \lambda_{L L}^{(V / T)}=\lambda_{R R}^{(V / T)}=-\lambda_{R L}^{(V / T)}=-\lambda_{L R}^{(V / T)}=\lambda_{A V / A T}
\end{aligned}
$$

These four combinations correspond to the pure right handed $(\mathrm{RH})$, left handed $(\mathrm{LH})$, vector/tensor and axial vector/axial tensor couplings respectively. The symmetry in the helicity amplitudes given in equations A.15 - A.18 and A.27 - A.30 renders the same new physics contributions for the combination (a) and (b).

\section{Color Singlet Vector Unparticles}

We study the effect of presence of color singlet unparticles on the three observables, namely, $\sigma^{t \bar{t}}, A_{F B}^{t \bar{t}}$ and $C^{t \bar{t}}$. The variation of these observables with the product of couplings is shown in figures 2, 3] and 4 respectively corresponding to the specific cases.

Since there is no interference of the color singlet FC vector unparticle with $\mathrm{QCD}$ and the squared term of the unparticle dominates over the interference with the electroweak sector, the behavior of observables for singlet unparticles are determined by the contribution of unparticle alone. For the $\mathrm{FC}$ vector singlet unparticle, the contribution of unparticle is given by 


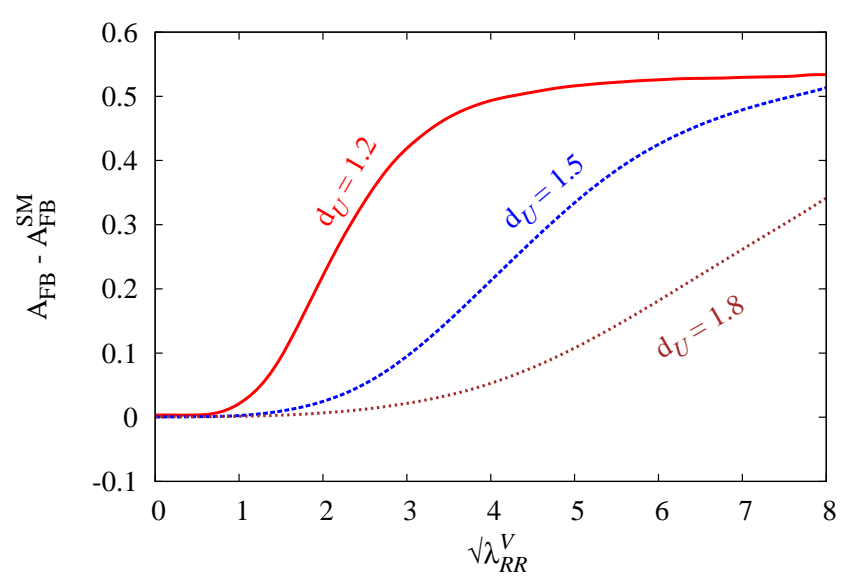

(a) $\lambda_{R R}^{V} \neq 0$ and $\lambda_{L R}^{V}=\lambda_{R L}=\lambda_{L L}^{V}=0$

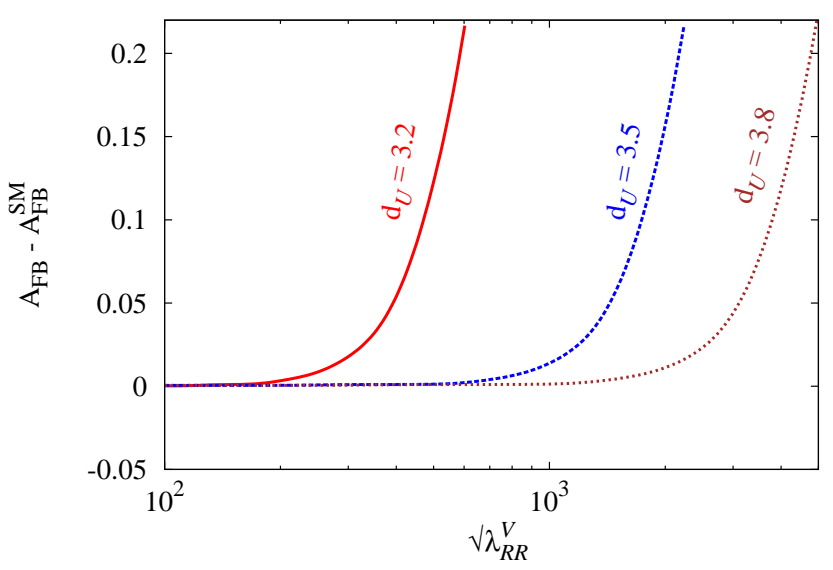

(b) $\lambda_{R R}^{V} \neq 0$ and $\lambda_{L R}^{V}=\lambda_{R L}^{V}=\lambda_{L L}^{V}=0$

FIG. 3: Variation of the $A_{F B}-A_{F B}^{S M}$ (the unparticle contribution to $A_{F B}^{t \bar{t}}$ ) with couplings $\sqrt{ } \lambda_{R R}^{V}$ (corresponding to the case (a) of the text) for color singlet flavor conserving vector unparticles for various value of $d_{\mathcal{U}}$ at fixed $\Lambda_{\mathcal{U}}=1$ TeV. This is

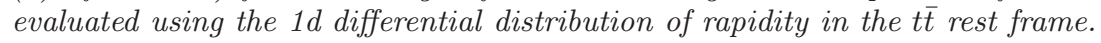

$$
\begin{aligned}
&\left|\mathcal{M}_{\mathrm{FCV}}^{\mathrm{unp}}\right|_{\text {same hel }}^{2}=B_{\mathrm{FCV}}^{\mathrm{unp}} {\left[\frac{1}{2}\left(1-\beta_{t}^{2}\right)\{(\right.} \\
&\left.\left.\left.\mid \mathcal{M}_{R R}^{V}+\lambda_{R L}^{V}\right)^{2}+\left(\lambda_{L L}^{V}+\lambda_{L R}^{V}\right)^{2}\right\} s_{\theta}^{2}\right] \\
&\left.\right|_{\text {opp hel }} ^{2}=B_{\mathrm{FCV}}^{\mathrm{unp}}\left[\frac{1}{2}\left\{\left(1+\beta_{t}^{2}\right)\left[\left(\lambda_{R R}^{V}\right)^{2}+\left(\lambda_{L L}^{V}\right)^{2}+\left(\lambda_{R L}^{V}\right)^{2}+\left(\lambda_{L R}^{V}\right)^{2}\right]+\left(1-\beta_{t}\right)^{2}\left(\lambda_{R R}^{V} \lambda_{R L}^{V}+\lambda_{L L}^{V} \lambda_{L R}^{V}\right)\right\}\left(1+c_{\theta}^{2}\right)\right. \\
&\left.+2 \beta_{t}\left(\left(\lambda_{R R}^{V}\right)^{2}+\left(\lambda_{L L}^{V}\right)^{2}-\left(\lambda_{R L}^{V}\right)^{2}-\left(\lambda_{L R}^{V}\right)^{2}\right) c_{\theta}\right]
\end{aligned}
$$

with $\quad B_{\mathrm{FCV}}^{\mathrm{unp}}=g_{s}^{2}\left(\frac{\hat{s}}{\Lambda_{\mathcal{U}}^{2}}\right)^{2\left(d_{\mathcal{U}}-1\right)}\left[\frac{A_{d_{\mathcal{U}}}}{2 \sin \left(d_{\mathcal{U}} \pi\right)}\right]^{2}$

We highlight following observations with regard to the spin correlation $C^{t \bar{t}}$ and charge asymmetry $A_{F B}^{t \bar{t}}$ :

1. $A_{F B}^{t \bar{t}}$ gets contribution only from the opposite helicity amplitudes which is proportional to $2 \beta_{t}\left[\left(\lambda_{R R}^{V}\right)^{2}+\left(\lambda_{L L}^{V}\right)^{2}-\left(\lambda_{R L}^{V}\right)^{2}-\left(\lambda_{L R}^{V}\right)^{2}\right]$. Hence $A_{F B}^{t \bar{t}}$ vanishes for $\beta_{t}=0$ (i.e. when top is produced at threshold) and also for $\lambda_{L L}^{V}=\lambda_{R R}^{V}= \pm \lambda_{L R}^{V}=$ $\pm \lambda_{R L}^{V}$ where $+(-)$ corresponds to case (c) and (d) respectively. However, the small contribution will come from the interference with electroweak part. We show the variation of $\sigma^{t \bar{t}}$ and $C^{t \bar{t}}$ for this case in figures $2 \mathrm{c}$ and $4 \mathrm{c}$

2. As $\beta_{t}$ varies from 0 to 1 we observe that the spincorrelation varies from a large negative value to a positive number. This is attributed to the fact that the opposite (same) helicity contribution increases (decreases) with increasing $\beta_{t}$. Also, the contribution to $\sigma^{t \bar{t}}, A_{F B}^{t \bar{t}}$ and $C^{t \bar{t}}$ decreases with increasing $d_{\mathcal{U}}$ for a given coupling because of the term $\Lambda^{\left(d_{\mathcal{U}}-1\right)}$ in the denominator.
3. $A_{F B}^{t \bar{t}}$ and $C^{t \bar{t}}$ initially increase quadratically with the product of couplings and then becomes constant as for the large couplings the unparticle squared term takes over the SM, resulting into the cancellation of the coupling dependence in the numerator and denominator of these observables.

4. The $C_{F B}^{t \bar{t}} \approx A_{F B}^{t \bar{t}}=A_{F B}^{t \bar{t}}$ (opposite helicity) for $\mathrm{FC}$ vector unparticles as the interference term of the unparticles with EW part is negligibly small.

These graphs exhibit that it is possible to get appreciable $A_{F B}^{t \bar{t}}$ (keeping the cross section and spin correlation in agreement with the experimental values) for the cases (a) and (b). This higher value of $A_{F B}^{t \bar{t}}$ for the first two cases can be attributed to the complete asymmetry in the left and right couplings. We find that for the other two cases (pure vector in case (c) and pure axial vector in case (d)) there is no parameter region that agrees with experimental value of $\sigma^{t \bar{t}}$ and at the same time giving appreciable $A_{F B}^{t \bar{t}}$.

In figures $2 \mathrm{~d}, 3 \mathrm{~b}$ and $4 \mathrm{~d}$, we show the variation of $\sigma^{t \bar{t}}$, 


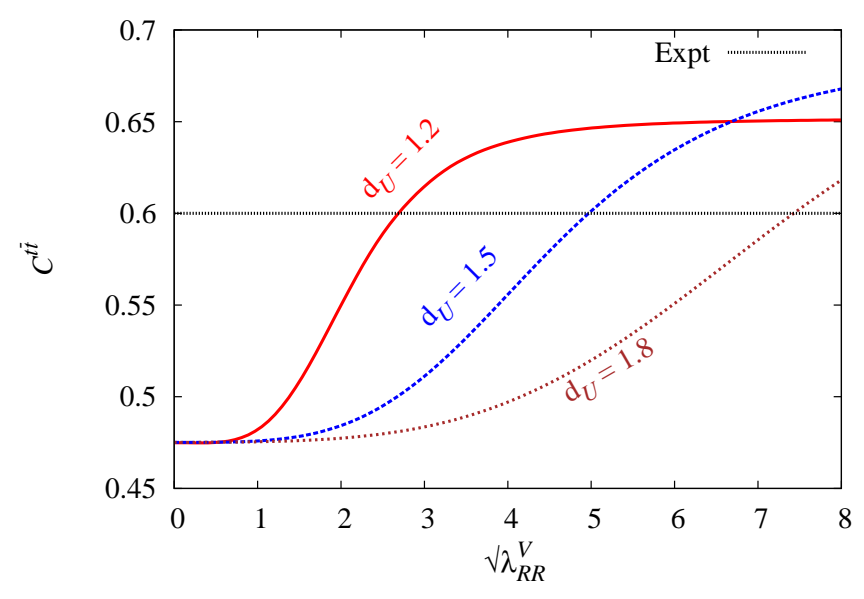

(a) $\lambda_{R R}^{V} \neq 0$ and $\lambda_{L R}^{V}=\lambda_{R L}^{V}=\lambda_{L L}^{V}=0$

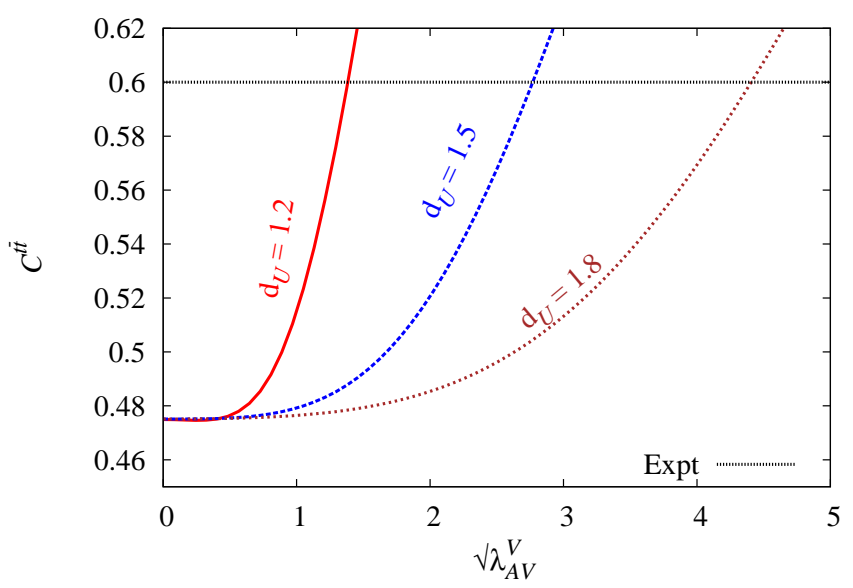

(c) $\lambda_{L L}^{V}=\lambda_{R R}^{V}=-\lambda_{R L}^{V}=-\lambda_{L R}^{V}=\lambda_{A V}$

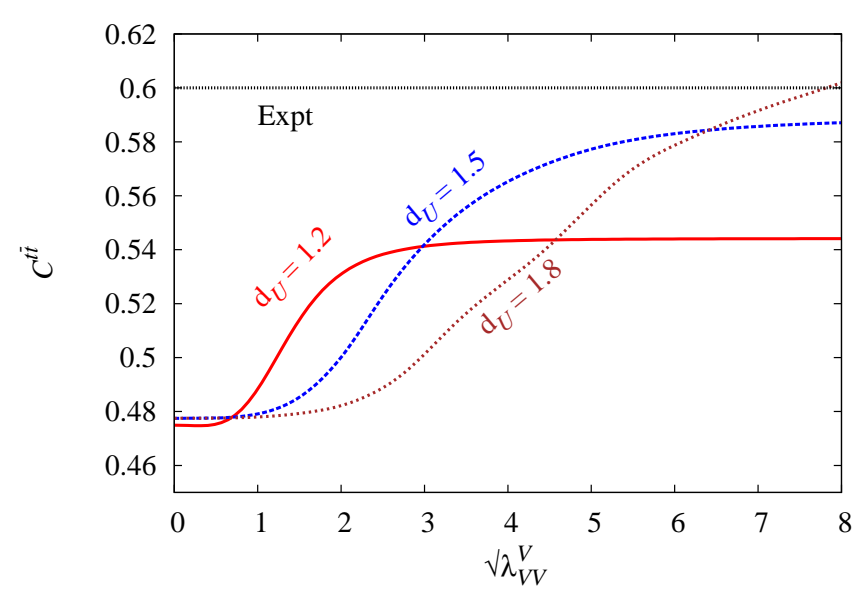

(b) $\lambda_{L L}^{V}=\lambda_{R R}^{V}=\lambda_{R L}^{V}=\lambda_{L R}^{V}=\lambda_{V V}$

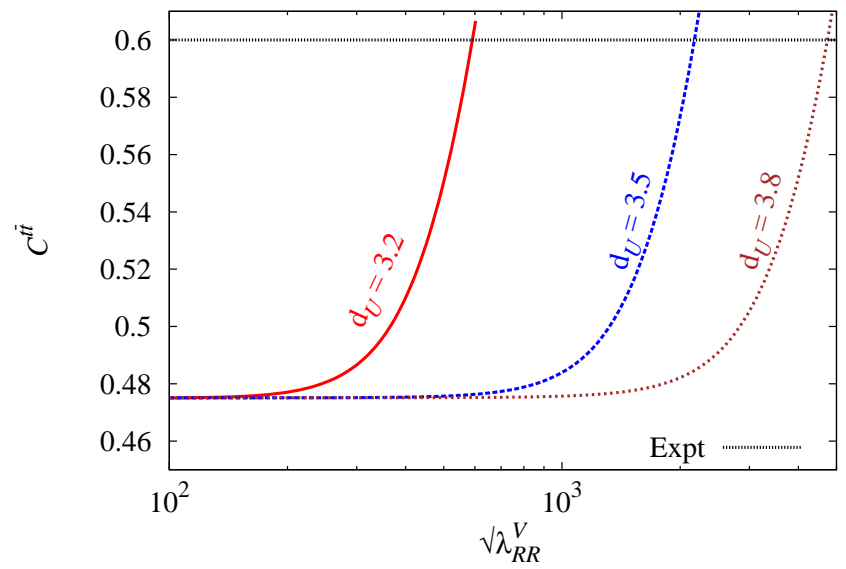

(d) $\lambda_{R R}^{V} \neq 0$ and $\lambda_{L R}^{V}=\lambda_{R L}^{V}=\lambda_{L L}^{V}=0$

FIG. 4: Variation of the spin-correlation coefficient $C^{t \bar{t}}$ with couplings $\sqrt{ } \lambda_{i j}^{V}$ for color singlet flavor conserving vector unparticles for various value of $d_{\mathcal{U}}$ at fixed $\Lambda_{\mathcal{U}}=1 \mathrm{TeV}$. The experimental value is depicted with a dot-dashed line at $0.60 \pm 0.50$ (stat) \pm 0.16 (syst) [28]. The panels 'a', 'b' and 'c' respectively correspond to the cases (a), (c) and (d) for the values of du in the range $1<d_{\mathcal{U}}<2$ while the panel 'd' gives the variation for case (a) with $d_{\mathcal{U}}$ in the range $3<d_{\mathcal{U}}<4$.

$A_{F B}^{t \bar{t}}$ and $C^{t \bar{t}}$ respectively with the coupling for $d_{\mathcal{U}}>3$ which is allowed by the unitarity of completely conformally invariant hidden sector. For such large values of $d_{\mathcal{U}}$ the unparticle effects are pronounced for very high values of couplings and hence the interference with electroweak sector plays a crucial role. It is to be noted that the SM contact terms induced at large scale can also become important in this region of parameter space.

\section{Color Octet Vector Unparticle}

Next we consider the possibility of vector unparticles being color octet with flavor conserving couplings. The variation of $\sigma^{t \bar{t}}, A_{F B}^{t \bar{t}}$ and $C^{t \bar{t}}$ with couplings are shown in the figures 516 and 7 respectively. The flavor conserving octet unparticles do not interfere with flavor singlet electroweak sector. Thus the nature of these numbers can be explained completely on the basis of the interplay of QCD and octet unparticle helicity amplitudes. From equations (18) and (19), it may be seen that the interference terms given in equation (23), can be rewritten as 


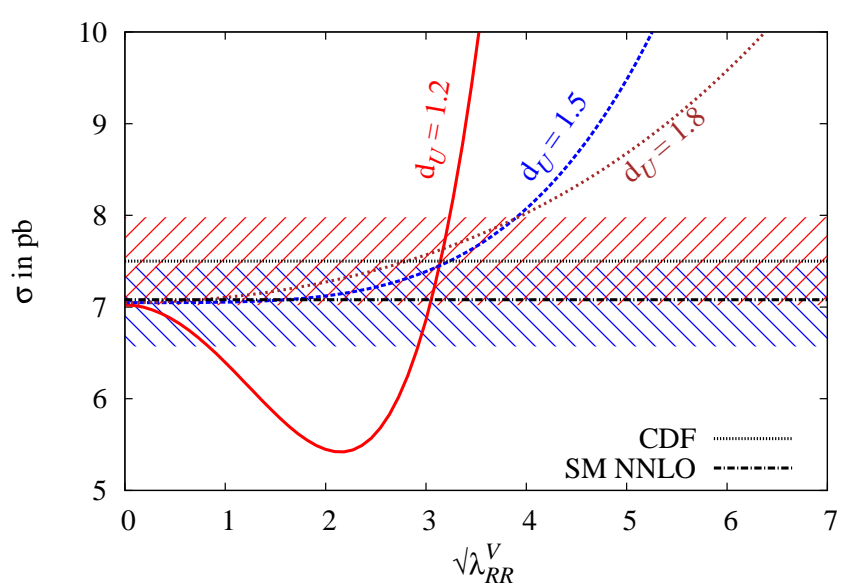

(a) $\lambda_{R R}^{V} \neq 0$ and $\lambda_{L R}^{V}=\lambda_{R L}^{V}=\lambda_{L L}^{V}=0$

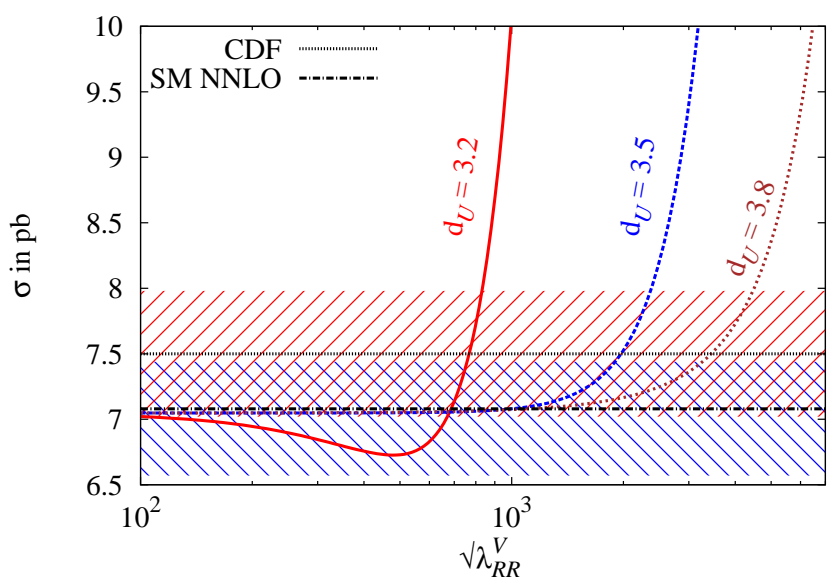

(c) $\lambda_{R R}^{V} \neq 0$ and $\lambda_{L R}^{V}=\lambda_{R L}^{V}=\lambda_{L L}^{V}=0$

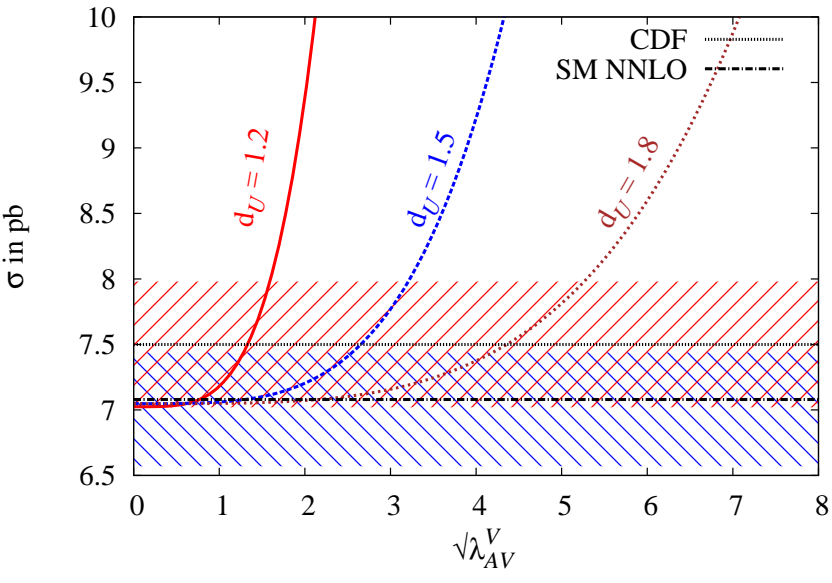

(b) $\lambda_{L L}^{V}=\lambda_{R R}^{V}=-\lambda_{R L}^{V}=-\lambda_{L R}^{V}=\lambda_{A V}^{V}$

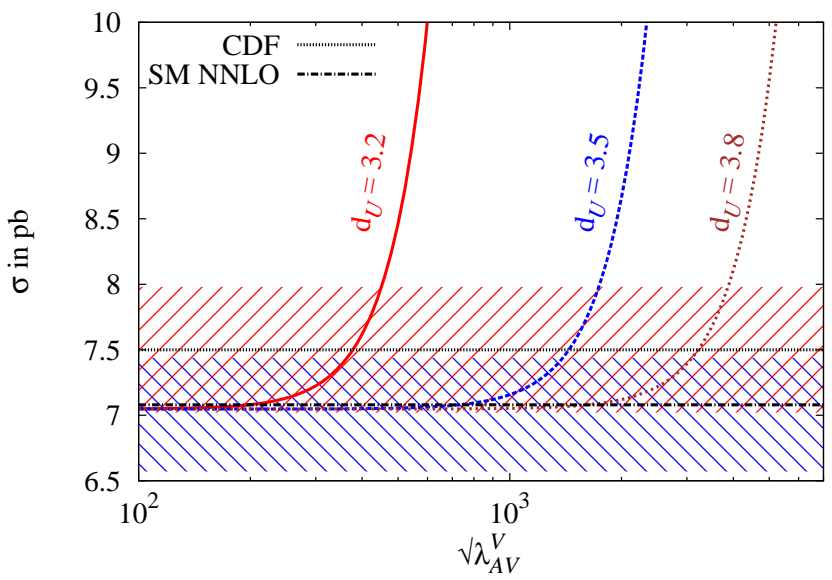

(d) $\lambda_{L L}^{V}=\lambda_{R R}^{V}=-\lambda_{R L}^{V}=-\lambda_{L R}^{V}=\lambda_{A V}^{V}$

FIG. 5: Variation of the cross-section $\sigma(p \bar{p} \rightarrow t \bar{t})$ with couplings $\sqrt{ } \lambda_{i j}^{V}$ for color octet flavor conserving vector unparticles corresponding to different values of $d_{\mathcal{U}}$ at fixed $\Lambda_{\mathcal{U}}=1 \mathrm{TeV}$. The upper dotted line with a red band depicts the cross-section $7.50 \pm 0.48 \mathrm{pb}$ from CDF (all channels) [1], while the lower dot-dashed line with a blue band show theoretical estimate $7.08 \pm 0.36$ $p b$ at $N N L O$ [4]. The panels ' $a$ ' and ' $b$ ' show the variation of $\sigma$ for the cases (a) and (d) of the text for the various values of $d_{\mathcal{U}}$ in the range $1<d_{\mathcal{U}}<2$ while the panels ' $c$ ' and 'd' give the same variation for the $d_{\mathcal{U}}$ values in the range $3<d_{\mathcal{U}}<4$.

$$
\begin{aligned}
& {\left[2 \mathcal{M}^{\mathrm{QCD}} \mathcal{R} e\left(\mathcal{M}_{\mathrm{FCV}}^{\mathrm{unp}}\right)\right]_{\text {same hel }}=2 B_{\mathrm{FCV}}^{\mathrm{int}}\left(\lambda_{L L}^{V}+\lambda_{L R}^{V}+\lambda_{R L}^{V}+\lambda_{R R}^{V}\right)\left(1-\beta_{t}^{2}\right) s_{\theta}^{2}} \\
& \text { and } \\
& \begin{aligned}
{\left[2 \mathcal{M}^{\mathrm{QCD}} \mathcal{R} e\left(\mathcal{M}_{\mathrm{FCV}}^{\mathrm{unp}}\right)\right]_{\text {opp hel }}=2 B_{\mathrm{FCV}}^{\mathrm{int}} } & {\left[\left(\lambda_{L L}^{V}+\lambda_{R R}^{V}+\lambda_{L R}^{V}+\lambda_{R L}^{V}\right)\left(1+c_{\theta}^{2}\right)\right.} \\
& \left.+2 \beta_{t}\left(\lambda_{L L}^{V}+\lambda_{R R}^{V}-\lambda_{R L}^{V}-\lambda_{L R}^{V}\right) c_{\theta}\right] \\
& B_{\mathrm{FCV}}^{\mathrm{int}}=g_{s}^{2}\left(\frac{\hat{s}}{\Lambda_{\mathcal{U}}^{2}}\right)^{\left(d_{\mathcal{U}}-1\right)} A_{d \mathcal{U}}\left[-\frac{\cot \left(d_{\mathcal{U}} \pi\right)}{2}\right]
\end{aligned}
\end{aligned}
$$

The $\left|\mathcal{M}_{\mathrm{FCV}}^{\text {unp }}\right|_{\text {same hel }}^{2}$ and $\left|M_{\mathrm{FCV}}^{\mathrm{opp}}\right|_{\text {same hel }}^{2}$ are same as given

that in equation (25). Analysing these expressions we observe 


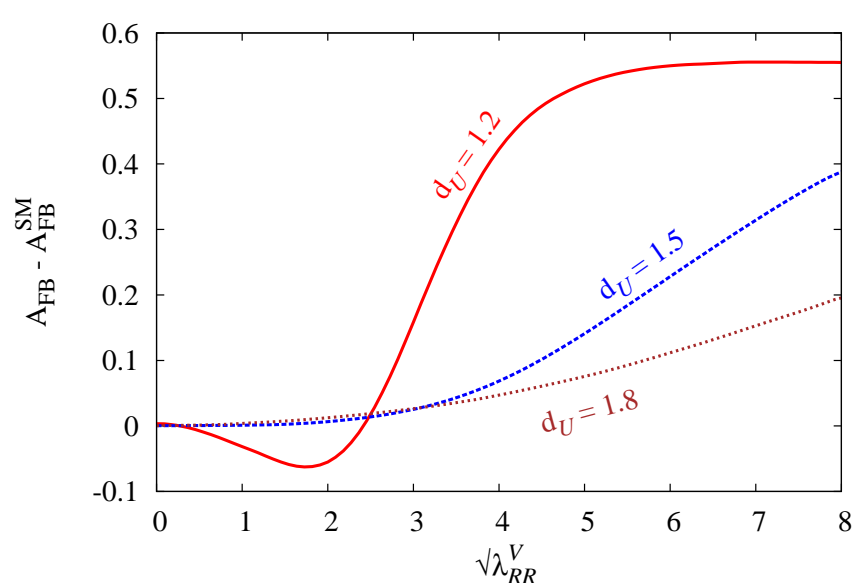

(a) $\lambda_{R R}^{V} \neq 0$ and $\lambda_{L R}^{V}=\lambda_{R L}^{V}=\lambda_{L L}^{V}=0$

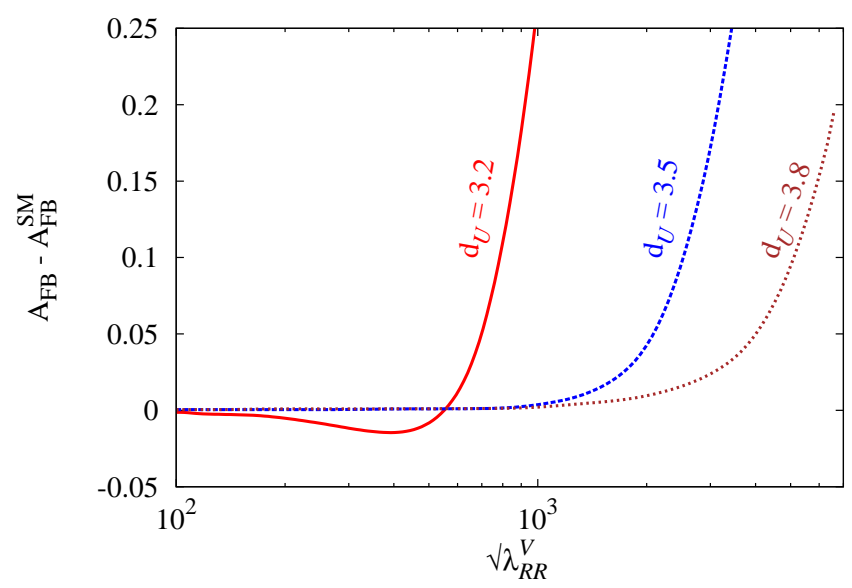

(c) $\lambda_{R R}^{V} \neq 0$ and $\lambda_{L R}^{V}=\lambda_{R L}^{V}=\lambda_{L L}^{V}=0$

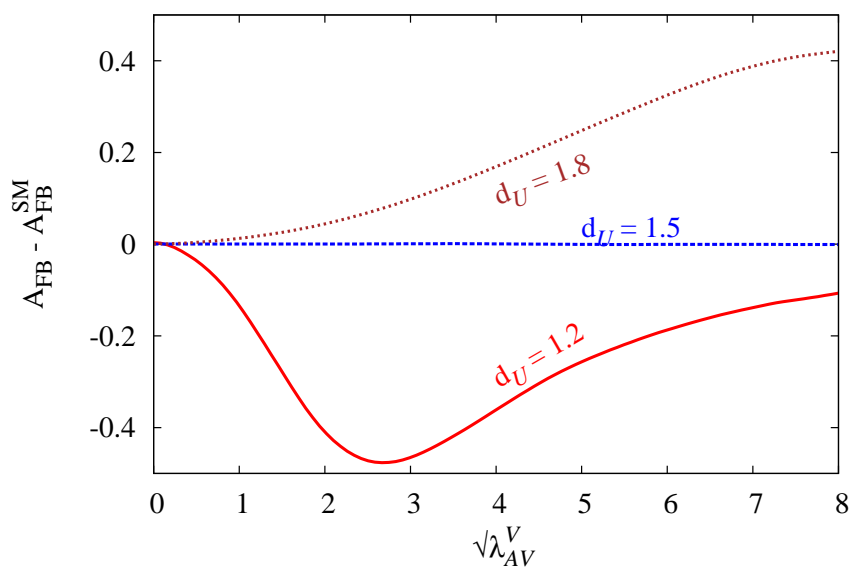

(b) $\lambda_{L L}^{V}=\lambda_{R R}^{V}=-\lambda_{R L}^{V}=-\lambda_{L R}^{V}=\lambda_{A V}^{V}$

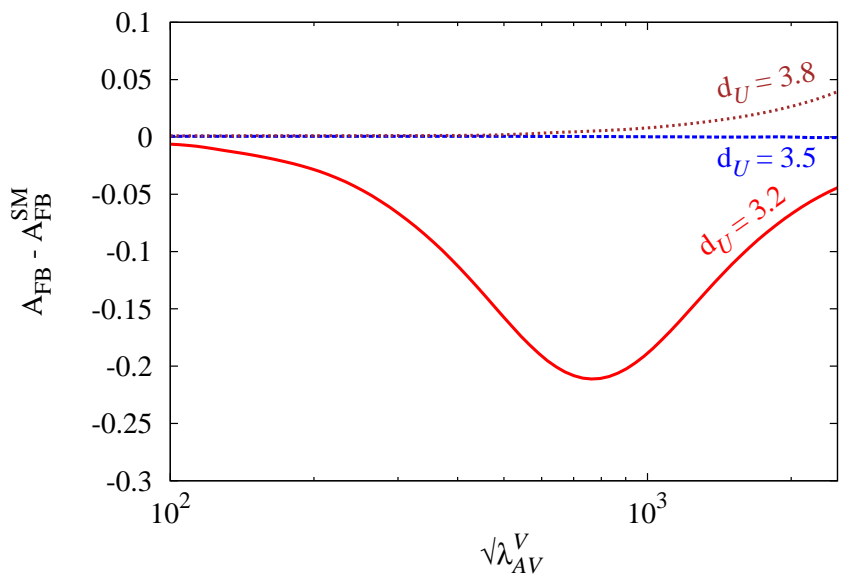

(d) $\lambda_{L L}^{V}=\lambda_{R R}^{V}=-\lambda_{R L}^{V}=-\lambda_{L R}^{V}=\lambda_{A V}^{V}$

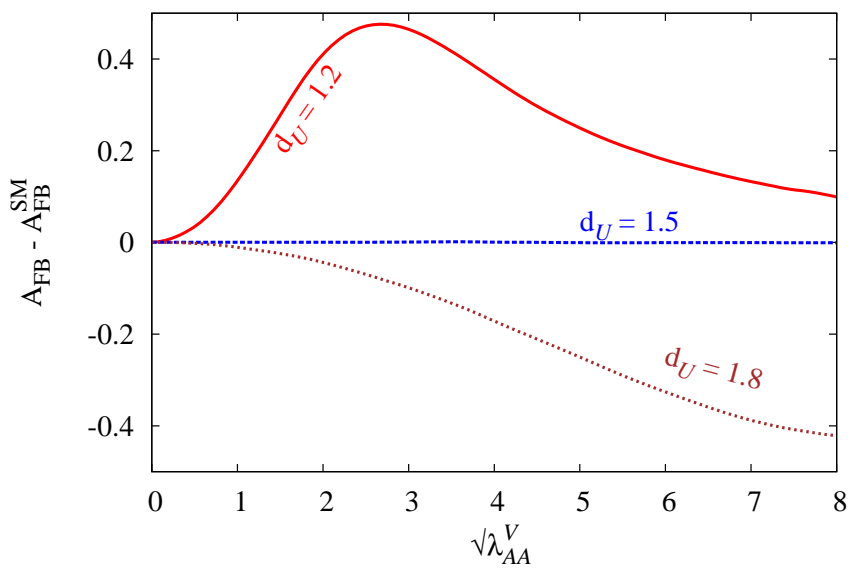

(e) $g_{L / R}^{\mathcal{U}_{V} \bar{q} q} g_{L / R}^{\mathcal{U}_{V} \bar{t} t}<0$, the choice of coupling combination favored by the non-universal axigluon models

FIG. 6: Variation of the $A_{F B}-A_{F B}^{S M}$ (the unparticle contribution to $A_{F B}^{t \bar{t}}$ ) with couplings $\sqrt{ } \lambda_{i j}^{V}$ for color octet flavor conserving vector unparticles for various values of $d_{\mathcal{U}}$ at fixed $\Lambda_{\mathcal{U}}=1 \mathrm{Te} V$ and for different coupling combinations mentioned in the text.

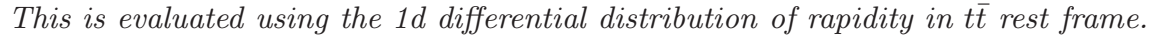

1. In the interference term, unlike in the spin correlation only the opposite helicity amplitudes 


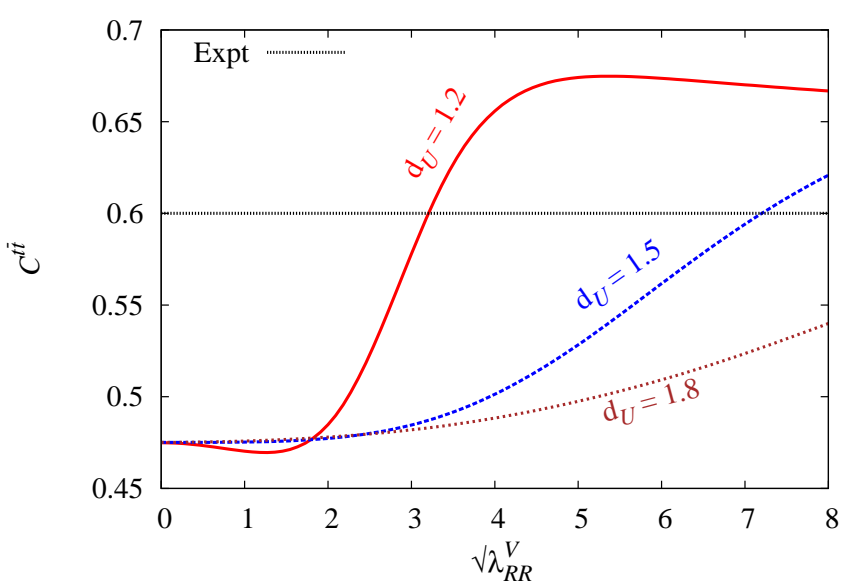

(a) $\lambda_{R R}^{V} \neq 0$ and $\lambda_{L R}^{V}=\lambda_{R L}^{V}=\lambda_{L L}^{V}=0$

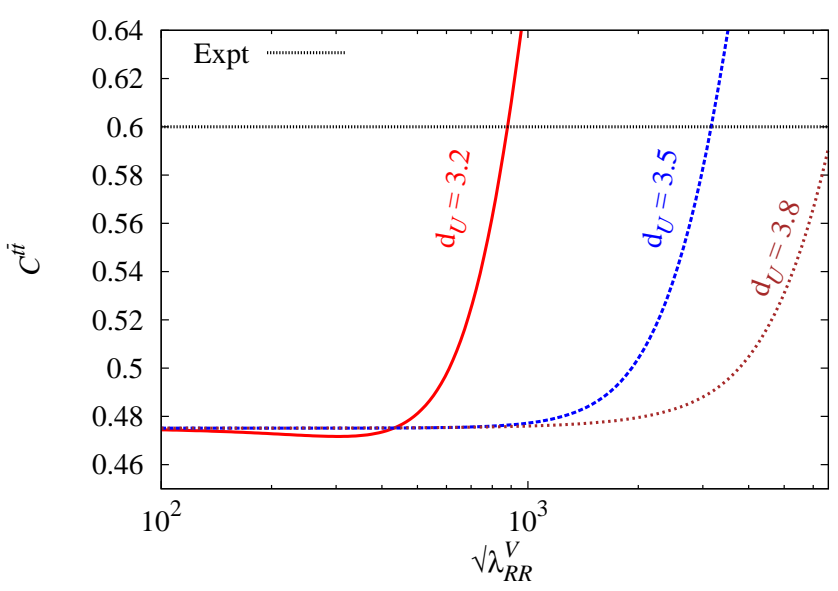

(c) $\lambda_{R R}^{V} \neq 0$ and $\lambda_{L R}^{V}=\lambda_{R L}^{V}=\lambda_{L L}^{V}=0$

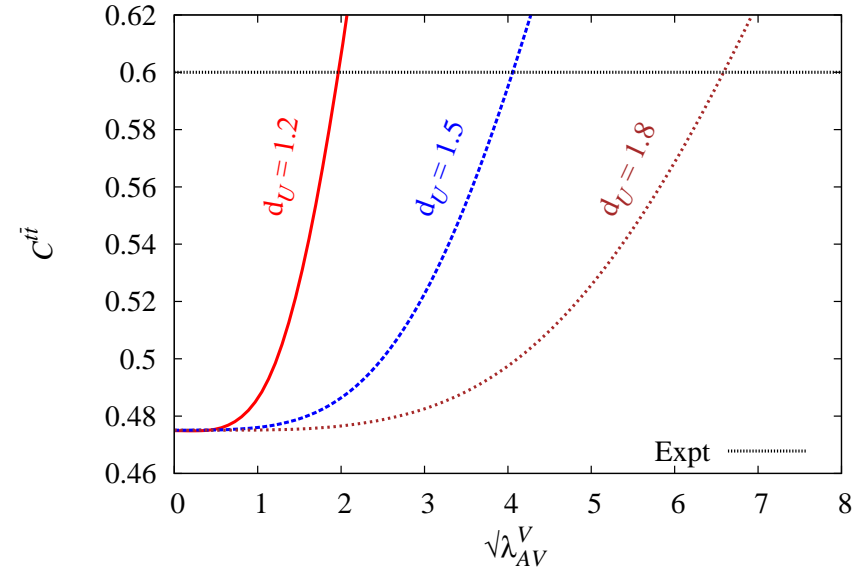

(b) $\lambda_{L L}^{V}=\lambda_{R R}^{V}=-\lambda_{R L}^{V}=-\lambda_{L R}^{V}=\lambda_{A V}^{V}$

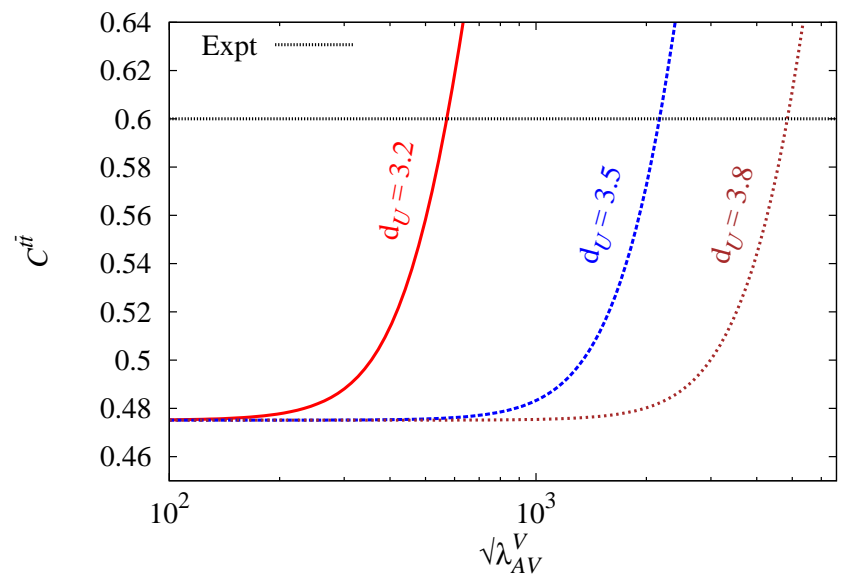

(d) $\lambda_{L L}^{V}=\lambda_{R R}^{V}=-\lambda_{R L}^{V}=-\lambda_{L R}^{V}=\lambda_{A V}^{V}$

FIG. 7: Variation of the spin-correlation coefficient $C^{t \bar{t}}$ evaluated in helicity basis with couplings $\sqrt{ } \lambda_{i j}^{V}$ for color octet flavor conserving vector unparticles for various value of $d_{\mathcal{U}}$ at fixed $\Lambda_{\mathcal{U}}=1$ TeV and for different combinations of couplings. The experimental value is depicted with a dot-dashed line at $0.60 \pm 0.50$ (stat) \pm 0.16 (syst) [28].

contributes to $A_{F B}^{t \bar{t}}$ which is proportional to $4 \beta_{t}\left(\lambda_{L L}^{V}+\lambda_{R R}^{V}-\lambda_{R L}^{V}-\lambda_{L R}^{V}\right)$. Hence $C_{F B}^{t \bar{t}}=$ $A_{F B}^{t \bar{t}}$.

2. The helicity amplitudes for unparticles and QCD are left-right symmetric. Therefore the variation of the observables with coupling products will be same for case (a) and case (b). We show the variation for $\sigma^{t \bar{t}}, A_{F B}^{t \bar{t}}$ and $C^{t \bar{t}}$ for the case (a) in figures 5a, 6a and $7 \mathrm{a}$ for the phenomenologically interesting range of $d_{\mathcal{U}}$ i.e. $1<d_{\mathcal{U}}<2$. The same variation for $3<d_{\mathcal{U}}<4$ (consistent with the conformally invariant sector) is shown in figures $5 \mathrm{c}, 6 \mathrm{c}$ and $7 \mathrm{c}$ respectively. The case (c) corresponds to pure vector couplings of unparticles with quarks which is similar to that of QCD. Hence negligible $A_{F B}^{t \bar{t}}$ is generated only from the squared term of the EW neutral current. The case (d) on the other hand generates appreciable $A_{F B}^{t \bar{t}}$ proportional to $16 \beta_{t} \lambda_{A V}^{V}$ due to the interference of vector (QCD) with axial vector unparticle sector. The variation for $\sigma^{t \bar{t}}, A_{F B}^{t \bar{t}}$ and $C^{t \bar{t}}$ for case (d) is shown in the figures $5 \mathrm{~b}, 6 \mathrm{~b}$ and $7 \mathrm{~b}$ for $1<d_{\mathcal{U}}<2$. The same variation for $3<d_{\mathcal{U}}<4$ (consistent with the conformally invariant sector) is shown in figures $5 \mathrm{~d}$, $6 \mathrm{~d}$ and $7 \mathrm{~d}$ respectively.

3. The sign of interference term is determined by the unparticle propagator which has a non-trivial phase dependence upon $d_{\mathcal{U}}$. We may write

$|\mathcal{M}|^{2}=\left|\mathcal{M}^{\mathrm{SM}}\right|^{2}+\delta\left[2 \mathcal{M}^{\mathrm{QCD}}\left|\mathcal{R} e\left(\mathcal{M}^{\mathrm{unp}}\right)\right|\right]$

where $\delta$ is negative for $n<d_{\mathcal{U}}<(n+1 / 2)$, zero for $d_{\mathcal{U}}=(n+1 / 2)$ and positive for $(n+1 / 2)<$ $d_{\mathcal{U}}<(n+1)$ due to the presence of $\cot \left(d_{\mathcal{U}} \pi\right)(n$ 
being an integer $\geq 1$ ). This effect is evident in the figures 5. We observe that due to the effect of the interference, the cross-section in presence of unparticles first decreases with increase in couplings and eventually increases with the onset of $\left|\mathcal{M}_{\text {unp }}\right|^{2}$ for a rather large couplings.

4. This effect is also well demonstrated for $A_{F B}^{t \bar{t}}$ in figures $6 \mathrm{~b}$ and $6 \mathrm{~d}$ corresponding to case (d) where there is no contribution to $A_{F B}^{t \bar{t}}$ from $\left|\mathcal{M}_{\text {unp }}\right|^{2}$. However for case (a), as seen from figures 6a and 6c the unparticle squared term overtakes the interference term and drives the $A_{F B}^{t \bar{t}}$ towards a positive value with a gradual increase in the coupling strength.

It is worthy to mention that for all axigluon models we require the light quark-axigluon and topaxigluon coupling to be of opposite sign in order to generate positive asymmetry. This is not necessary with unparticles. However, if one chooses couplings in this non-universal way, then $A_{F B}^{t \bar{t}}$ will pick up extra negative sign as shown in figure 6e for $1<d_{\mathcal{U}}<2$. Even for $d_{\mathcal{U}}>3$ one obtains large positive asymmetry for $3<d_{\mathcal{U}}<3.5$ for this choice of couplings.

Thus with octet vector unparticle sector having pure right or left handed couplings or even with axial vector couplings, there is a region in parameter space (e.g. case(a) with $d_{\mathcal{U}}=1.2$ and case (d) with $d_{\mathcal{U}}=1.8$ ) where it is possible to get appreciable positive $A_{F B}^{t \bar{t}}$ keeping the $t \bar{t}$ production cross-section and spin correlation consistent with experimental measurement. For higher $d_{\mathcal{U}}$ consistent with the unitarity bound of completely conformally invariant sector, i.e. $d_{\mathcal{U}}>3$, not enough positive $A_{F B}^{t \bar{t}}$ is obtained for any $d_{\mathcal{U}}$ and coupling consistent with the $\sigma^{t \bar{t}}$.

Chen et al [16] have calculated $A_{F B}^{t \bar{t}}$ in the lab frame for the same case (for $\mathrm{FC}$ vector octet unparticles with pure right handed coupling). Using the definition of equation. (1) and for an identical choice of parameters our cross-section and $A_{F B}^{t \bar{t}}$ are in agreement with them.

\section{FC Color Singlet Tensor Unparticle}

As in the case of vector unparticles, the $s$ channel process through FC color singlet tensor unparticles interferes with the EW neutral current but not with the QCD. Therefore pure unparticle amplitude (i.e. $\left|\mathcal{M}^{\text {unp }}\right|^{2}$ ) determines the behavior of the observables. The left-right symmetry in helicity amplitudes results in almost identical behaviour for the observables corresponding to the cases (a) and (b) which is similar to FC vector unparticles. The same and opposite helicity contributions to $\left|\mathcal{M}^{\text {unp }}\right|^{2}$ are given by

$$
\begin{aligned}
\left|\mathcal{M}_{\mathrm{FCT}}^{\text {unp }}\right|_{\text {same hel }}^{2}= & \mathcal{B}_{\mathrm{FCT}}^{\text {unp }}\left[2 \beta_{t}^{2}\left(1-\beta_{t}^{2}\right)\left\{\left(\lambda_{R R}^{T}+\lambda_{R L}^{T}\right)^{2}+\left(\lambda_{L L}^{T}+\lambda_{L R}^{T}\right)^{2}\right\} s_{\theta}^{2} c_{\theta}^{2}\right] \\
\left|\mathcal{M}_{\mathrm{FCT}}^{\text {unp }}\right|_{\text {opp hel }}^{2}= & \frac{1}{2} \beta_{t}^{2} \mathcal{B}_{\mathrm{FCT}}^{\text {unp }}\left[\left\{\left(1+\beta_{t}^{2}\right)\left(\left(\lambda_{R R}^{T}\right)^{2}+\left(\lambda_{L L}^{T}\right)^{2}+\left(\lambda_{R L}^{T}\right)^{2}+\left(\lambda_{L R}^{T}\right)^{2}\right)\right.\right. \\
& \left.+2\left(1-\beta_{t}^{2}\right)\left(\lambda_{R R}^{T} \lambda_{R L}^{T}+\lambda_{L L}^{T} \lambda_{L R}^{T}\right)\right\}\left(1-3 c_{\theta}^{2}+4 c_{\theta}^{4}\right) \\
& \left.-4 \beta_{t}\left(\left(\lambda_{R R}^{T}\right)^{2}+\left(\lambda_{L L}^{T}\right)^{2}-\left(\lambda_{R L}^{T}\right)^{2}-\left(\lambda_{L R}^{T}\right)^{2}\right)\left(1-2 c_{\theta}^{2}\right) c_{\theta}\right] \\
\text { where } \quad \mathcal{B}_{\mathrm{FCT}}^{\text {unp }}= & \frac{1}{16} \mathcal{C}_{f}^{\mathcal{U}_{T}^{\mathrm{FC}}}\left[g_{s}^{2}\left(\frac{\hat{s}}{\Lambda_{\mathcal{U}}^{2}}\right)^{2 d_{\mathcal{U}}}\left[\frac{A_{d_{\mathcal{U}}}}{2 \sin \left(d_{\mathcal{U}} \pi\right)^{2}}\right]^{2}\left(d_{\mathcal{U}}{ }^{2}\right)\left(d_{\mathcal{U}}-1\right)^{2}\right]
\end{aligned}
$$

The variation of the observables cross section $\sigma^{t \bar{t}}$ and spin correlation $C^{t \bar{t}}$ with $\lambda_{i j}^{T}$ for different cases is shown in figures 8 and 9 respectively. On comparing with pure vector case we observe that an additional $\beta_{t}$ factor in the helicity amplitudes suppresses the $A_{F B}^{t \bar{t}}$ for case (a) or (b) in the range of parameters which keeps the cross section and spin correlation $C^{t \bar{t}}$ in agreement with the allowed experimental values. Cases (c) and (d) do not give any contribution to $A_{F B}^{t \bar{t}}$. Moreover, the order of couplings involved are much larger (keeping the $\sigma^{t \bar{t}}$ within experimental limits) compared to vector unparticle case because the overall coupling in tensor case have extra factor of $\Lambda$ in the denominator. Unitarity bounds the $d_{\mathcal{U}}^{T}$ values for conformally invariant symmetric tensor to be $d_{\mathcal{U}}^{T}>4$. However, for such values of $d_{\mathcal{U}}^{T}$ the unparticle effects are highly subdued as may be seen from figures 8 and 9 ]

\section{Color Octet Tensor Unparticle}

The FC tensor octet unparticles do not interfere with electroweak neutral sector like the vector unparticles and the behavior of the plots (shown in Figs. 10/11 and 12) is 


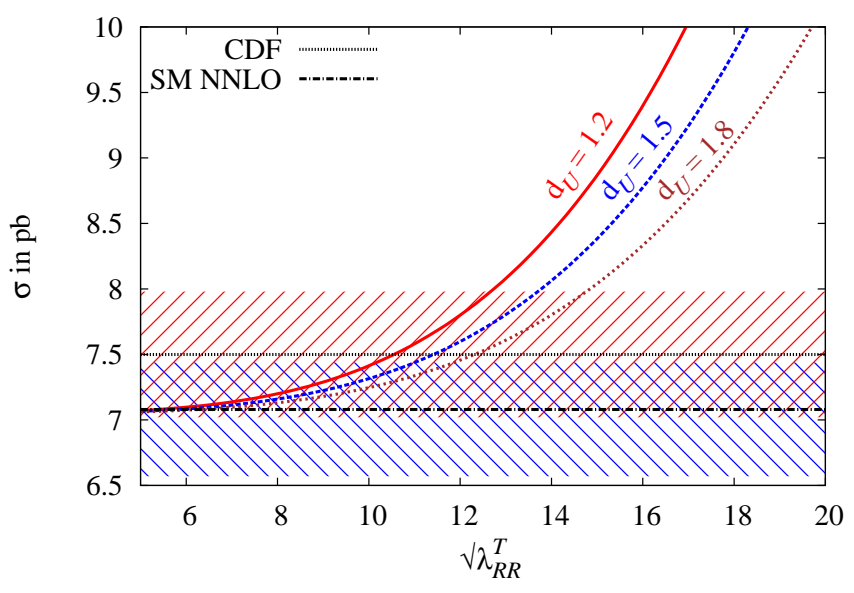

(a) $\lambda_{R R}^{T} \neq 0$ and $\lambda_{L R}^{T}=\lambda_{R L}^{T}=\lambda_{L L}^{T}=0$

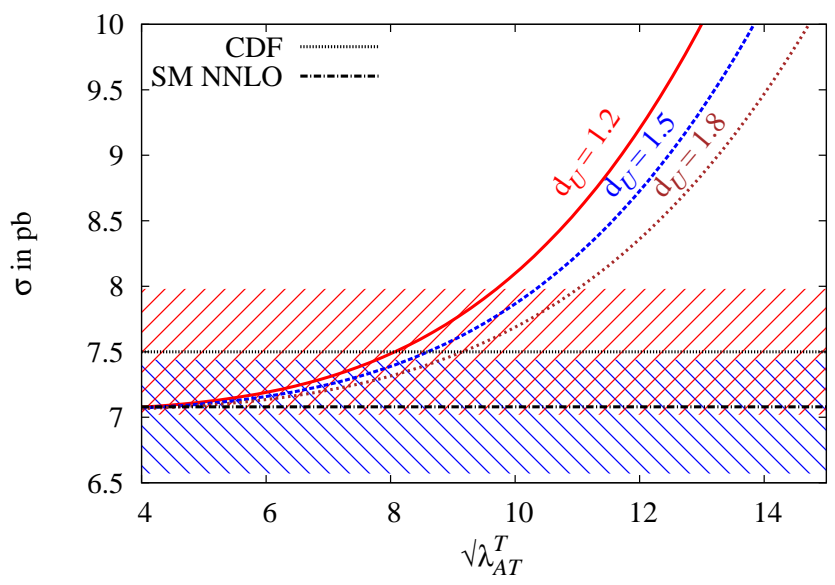

(c) $\lambda_{L L}^{T}=\lambda_{R R}^{T}=-\lambda_{R L}^{T}=-\lambda_{L R}^{T}=\lambda_{A T}^{T}$

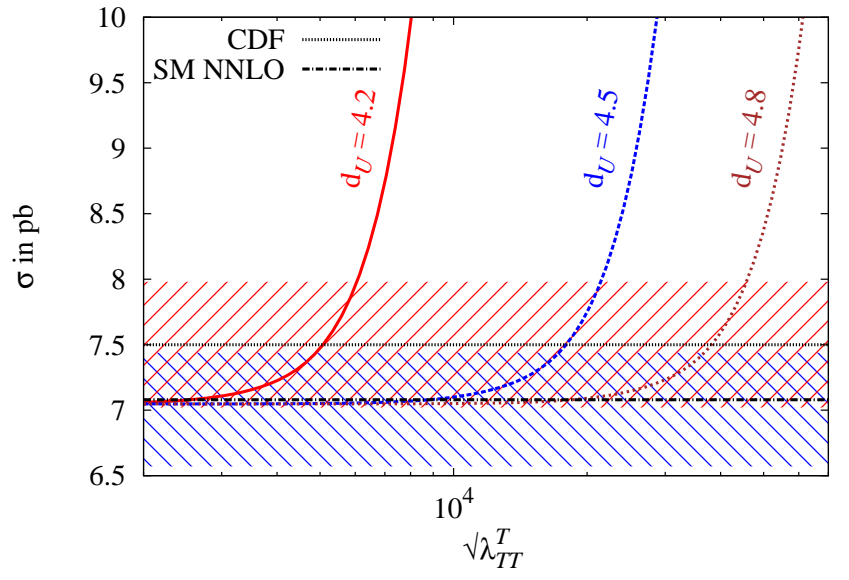

(e) $\lambda_{L L}^{T}=\lambda_{R R}^{T}=\lambda_{R L}^{T}=\lambda_{L R}^{T}=\lambda_{T T}^{T}$

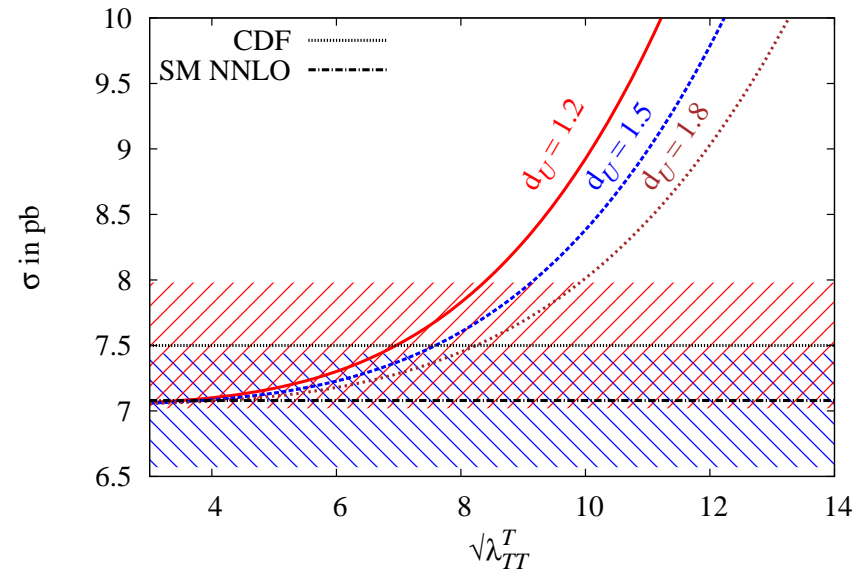

(b) $\lambda_{L L}^{T}=\lambda_{R R}^{T}=\lambda_{R L}^{T}=\lambda_{L R}^{T}=\lambda_{T T}^{T}$

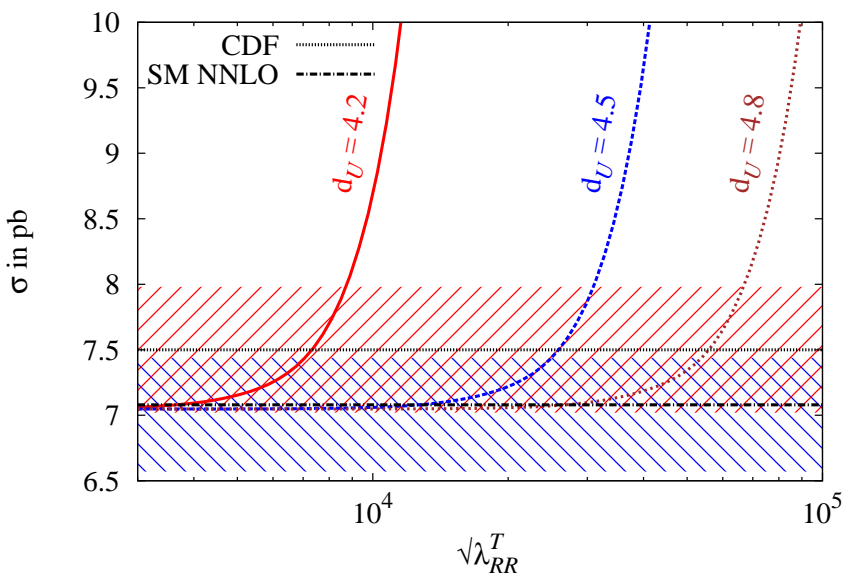

(d) $\lambda_{R R}^{T} \neq 0$ and $\lambda_{L R}^{T}=\lambda_{R L}^{T}=\lambda_{L L}^{T}=0$

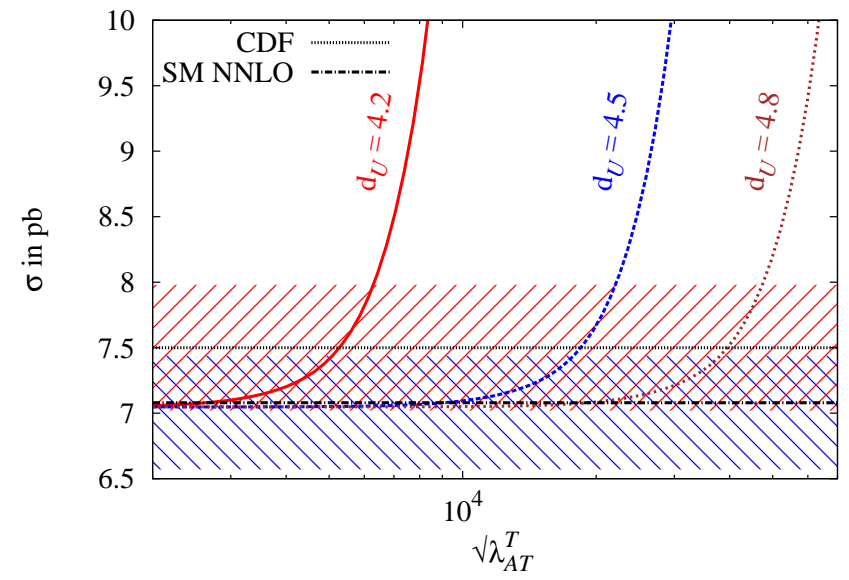

(f) $\lambda_{L L}^{T}=\lambda_{R R}^{T}=-\lambda_{R L}^{T}=-\lambda_{L R}^{T}=\lambda_{A T}$

FIG. 8: Variation of the cross-section $\sigma(p \bar{p} \rightarrow t \bar{t})$ with couplings $\sqrt{ } \lambda_{i j}^{T}$ for color singlet flavor conserving tensor unparticles corresponding to different values of $d_{\mathcal{U}}$ at fixed $\Lambda_{\mathcal{U}}=1$ TeV and for different combinations of couplings mentioned in the text . The upper dotted line with a red band depicts the cross-section $7.50 \pm 0.48$ pb from CDF (all channels) [1], while the lower dot-dashed line with a blue band show theoretical estimate $7.08 \pm 0.36 \mathrm{pb}$ at NNLO [4] .

almost entirely determined by its interference with QCD. The same helicity and opposite helicity contributions to 


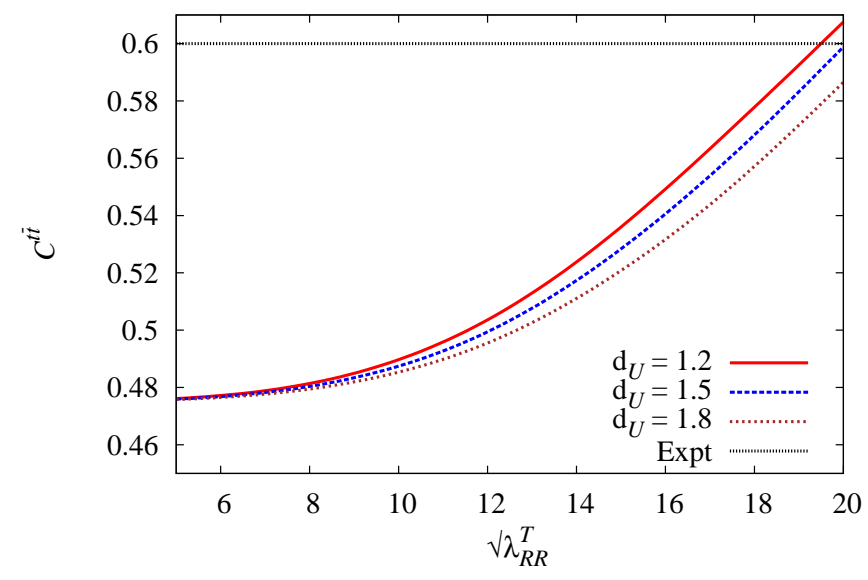

(a) $\lambda_{R R}^{T} \neq 0$ and $\lambda_{L R}^{T}=\lambda_{R L}^{T}=\lambda_{L L}^{T}=0$

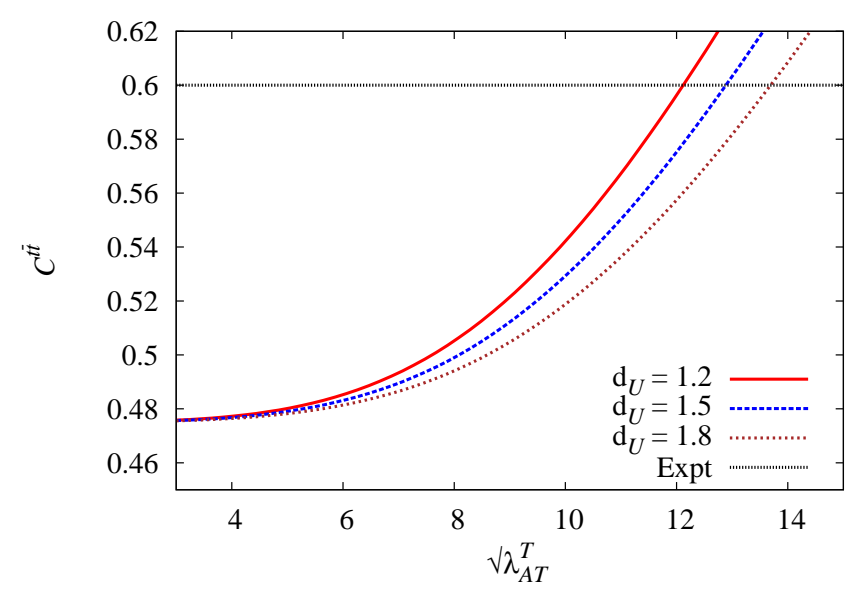

(c) $\lambda_{L L}^{T}=\lambda_{R R}^{T}=-\lambda_{R L}^{T}=-\lambda_{L R}^{T}=\lambda_{A T}$

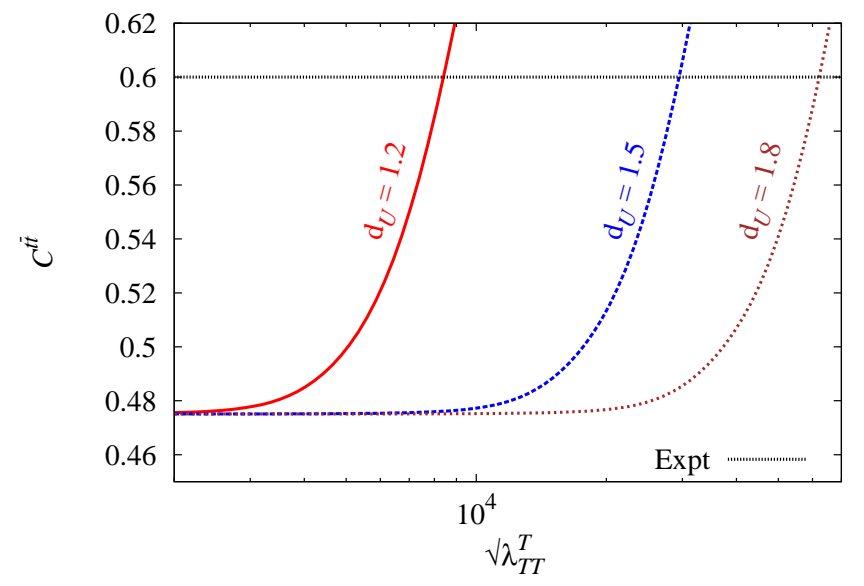

(e) $\lambda_{L L}^{T}=\lambda_{R R}^{T}=\lambda_{R L}^{T}=\lambda_{L R}^{T}=\lambda_{T T}^{T}$

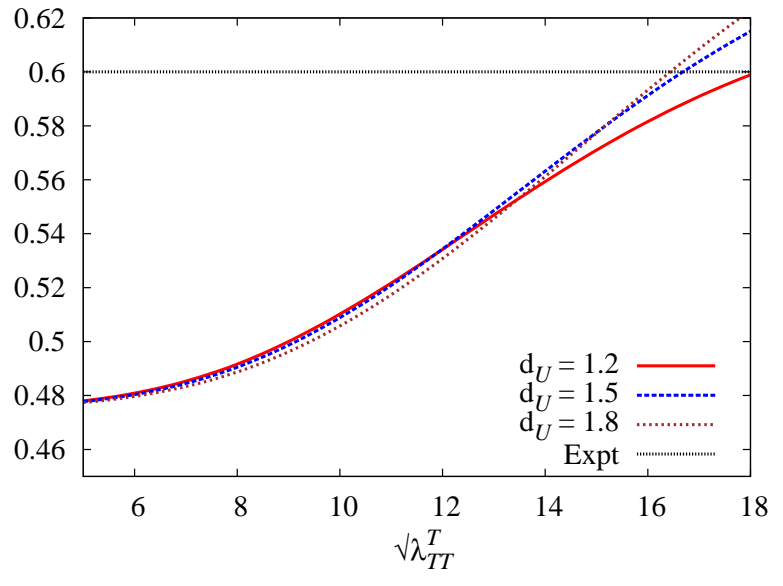

(b) $\lambda_{L L}^{T}=\lambda_{R R}^{T}=\lambda_{R L}^{T}=\lambda_{L R}^{T}=\lambda_{T T}^{T}$

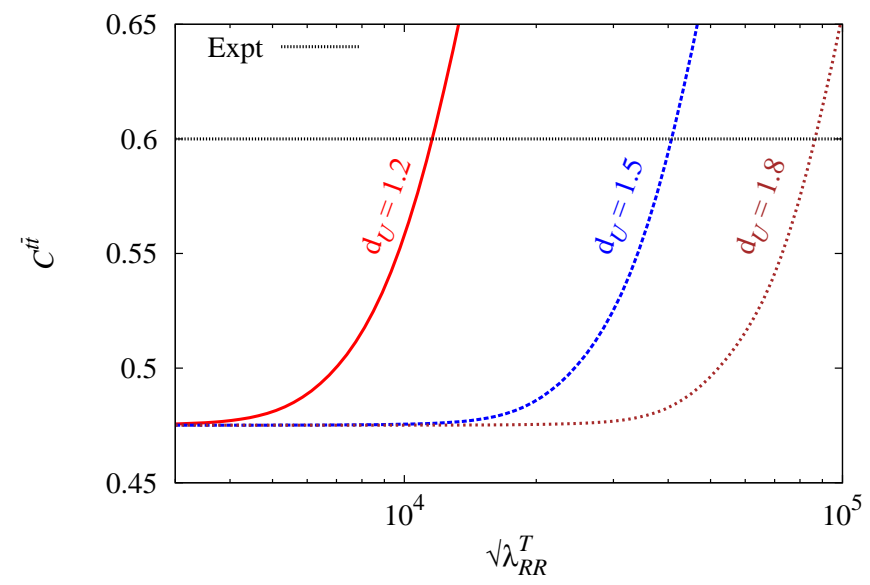

(d) $\lambda_{R R}^{T} \neq 0$ and $\lambda_{L R}^{T}=\lambda_{R L}^{T}=\lambda_{L L}^{T}=0$

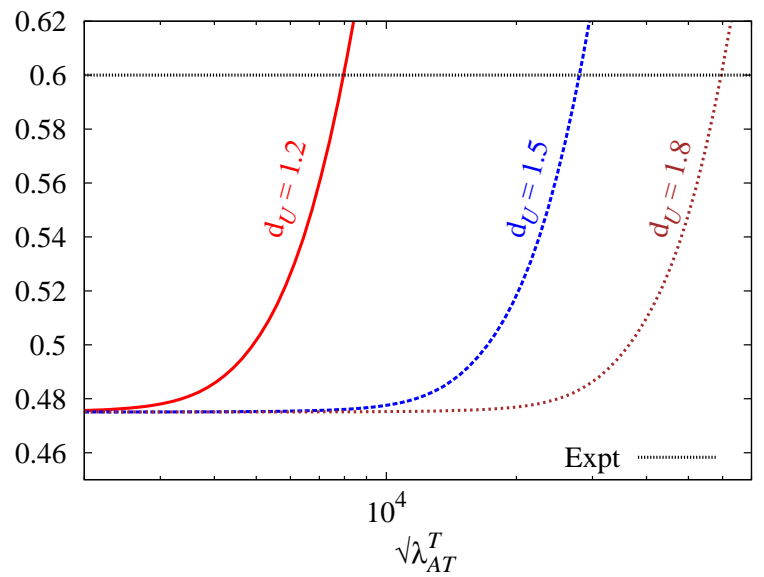

(f) $\lambda_{L L}^{T}=\lambda_{R R}^{T}=-\lambda_{R L}^{T}=-\lambda_{L R}^{T}=\lambda_{A T}$

FIG. 9: Variation of the spin-correlation coefficient $C^{t \bar{t}}$ evaluated in helicity basis with couplings $\sqrt{ } \lambda^{T}$ for color singlet flavor conserving tensor unparticles for various value of $d_{\mathcal{U}}$ at fixed $\Lambda_{\mathcal{U}}=1$ TeV and for different combinations of couplings. The experimental value is depicted with a dot-dashed line at $0.60 \pm 0.50$ (stat) \pm 0.16 (syst) [28]. 


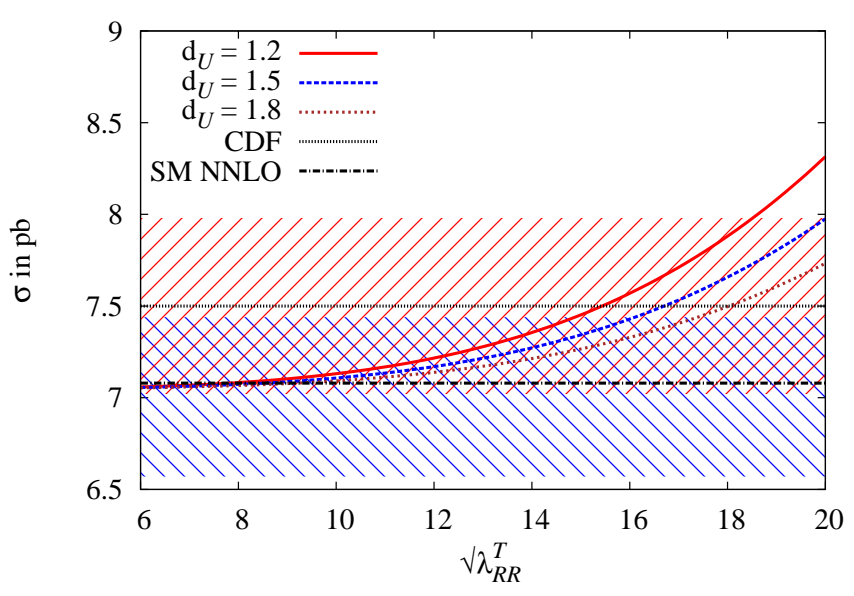

(a) $\lambda_{R R}^{T} \neq 0$ and $\lambda_{L R}^{T}=\lambda_{R L}^{T}=\lambda_{L L}^{T}=0$

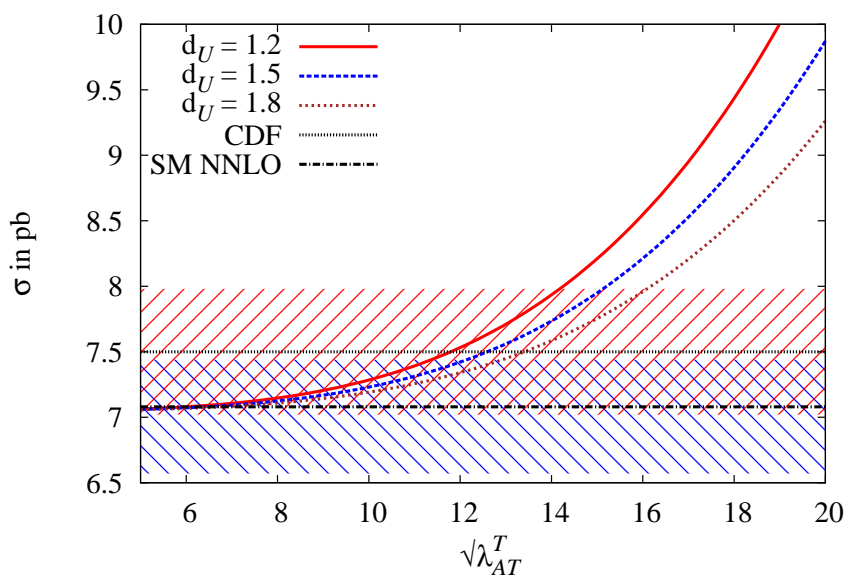

(c) $\lambda_{L L}^{T}=\lambda_{R R}^{T}=-\lambda_{R L}^{T}=-\lambda_{L R}^{T}=\lambda_{A T}$

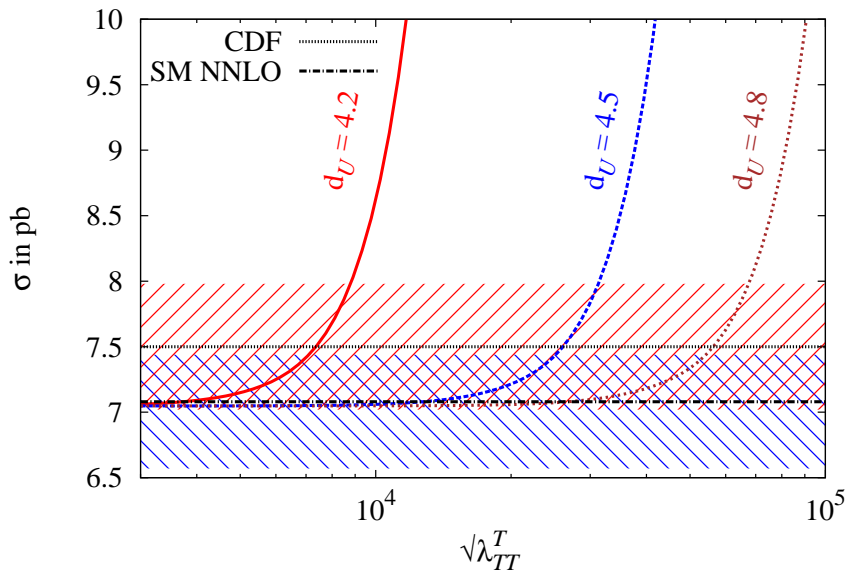

(e) $\lambda_{L L}^{T}=\lambda_{R R}^{T}=\lambda_{R L}^{T}=\lambda_{L R}^{T}=\lambda_{T T}^{T}$

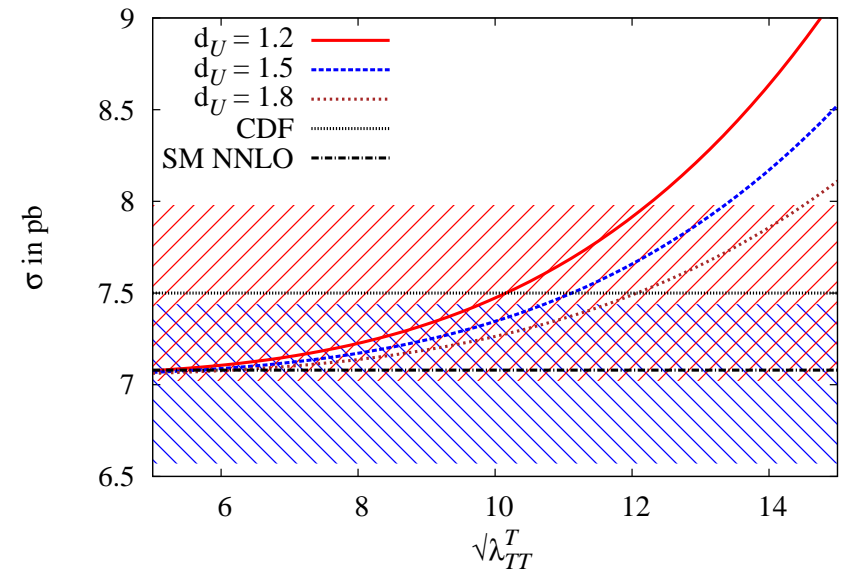

(b) $\lambda_{L L}^{T}=\lambda_{R R}^{T}=\lambda_{R L}^{T}=\lambda_{L R}^{T}=\lambda_{T T}^{T}$

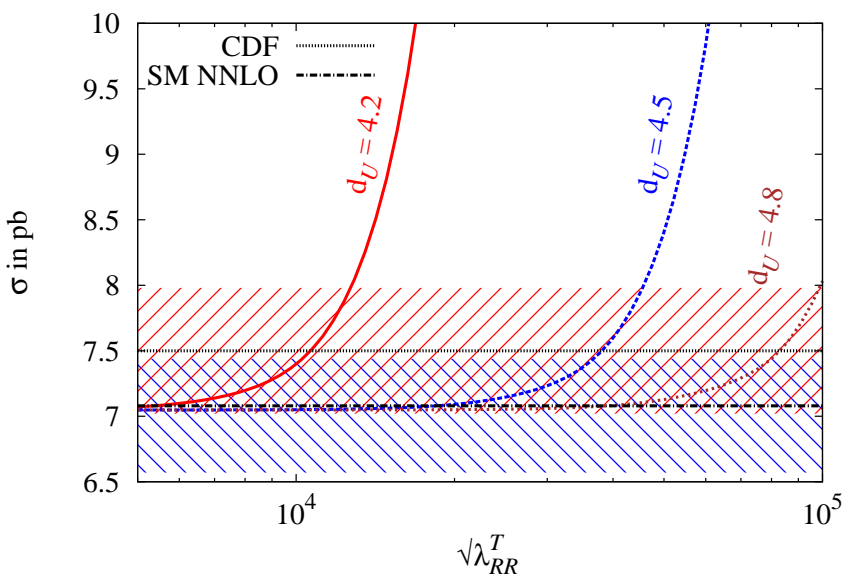

(d) $\lambda_{R R}^{T} \neq 0$ and $\lambda_{L R}^{T}=\lambda_{R L}^{T}=\lambda_{L L}^{T}=0$

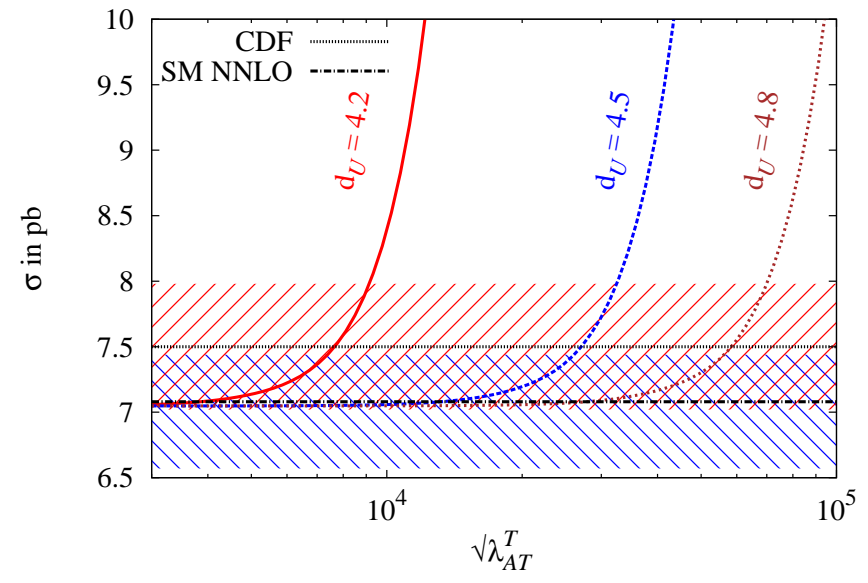

(f) $\lambda_{L L}^{T}=\lambda_{R R}^{T}=-\lambda_{R L}^{T}=-\lambda_{L R}^{T}=\lambda_{A T}$

FIG. 10: Variation of the cross-section $\sigma(p \bar{p} \rightarrow t \bar{t})$ with couplings $\sqrt{ } \lambda^{T}$ for color octet flavor conserving tensor unparticles corresponding to different values of $d_{\mathcal{U}}$ at fixed $\Lambda_{\mathcal{U}}=1 \mathrm{TeV}$. The upper dotted line with a red band depicts the cross-section $7.50 \pm 0.48 \mathrm{pb}$ from CDF (all channels) [1], while the lower dot-dashed line with a blue band show theoretical estimate $7.08 \pm 0.36$ pb at $N N L O$ [4]. 


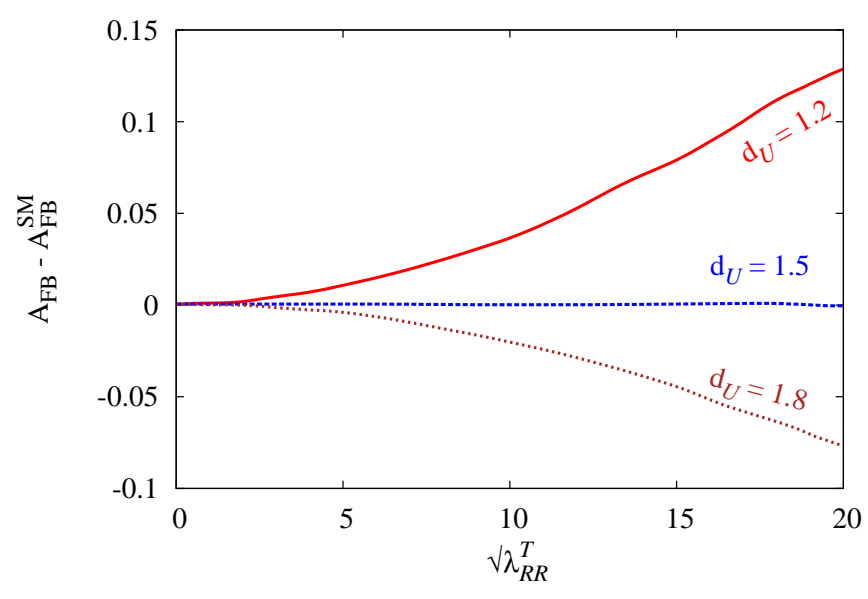

(a) $\lambda_{R R}^{T} \neq 0$ and $\lambda_{L R}^{T}=\lambda_{R L}^{T}=\lambda_{L L}^{T}=0$

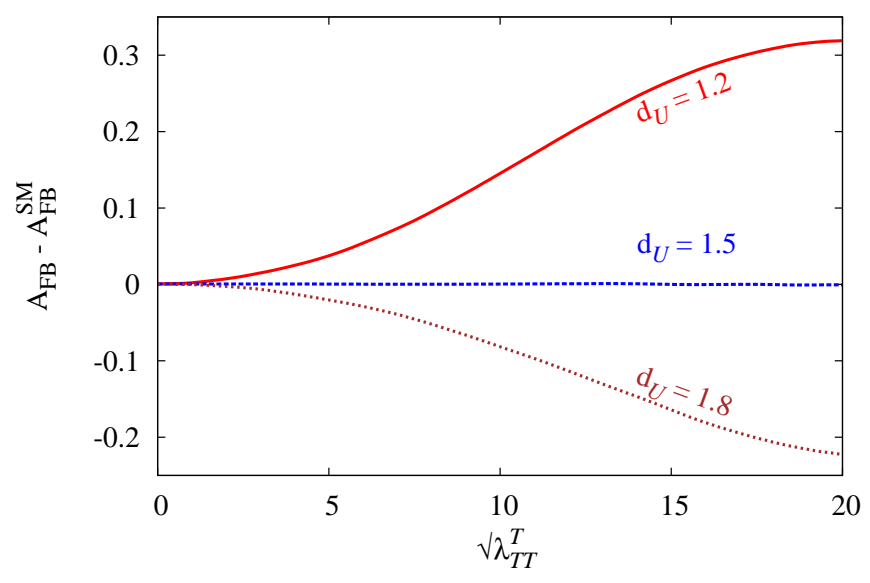

(b) $\lambda_{L L}^{T}=\lambda_{R R}^{T}=\lambda_{R L}^{T}=\lambda_{L R}^{T}=\lambda_{T T}^{T}$

FIG. 11: Variation of the $A_{F B}-A_{F B}^{S M}$ (the unparticle contribution to $A_{F B}^{t \bar{t}}$ ) with couplings $\sqrt{ } \lambda_{i j}^{T}$ for color octet flavor conserving tensor unparticles for various values of $d_{\mathcal{U}}$ for a fixed $\Lambda=1 T e V$. This is evaluated using the 1d differential distribution of rapidity in $t \bar{t}$ rest frame. The panels ' $a$ ' and ' $b$ ' correspond the cases (a) and (c) of the text.

interference with QCD are given by

$$
\begin{aligned}
\left.2 \mathcal{M}^{\mathrm{QCD}} \mathcal{R}\left(\mathcal{M}^{\text {unp }}\right)\right|_{\text {same hel }}= & -4 \mathcal{B}_{T}^{\text {int }}\left(\lambda_{L L}^{T}+\lambda_{L R}^{T}+\lambda_{R L}^{T}+\lambda_{R R}^{T}\right) \beta_{t}\left(1-\beta_{t}^{2}\right) s_{\theta}^{2} c_{\theta} \\
\left.2 \mathcal{M}^{\mathrm{QCD}} \mathcal{R}\left(\mathcal{M}^{\text {unp }}\right)\right|_{\text {opp hel }}= & -2 \mathcal{B}_{T}^{\text {int }} \beta_{t}\left[\beta_{t}\left(\lambda_{L L}^{T}+\lambda_{R R}^{T}-\lambda_{L R}^{T}-\lambda_{R L}^{T}\right)\left(3 c_{\theta}^{2}-1\right)\right. \\
& \left.+2\left(\lambda_{L L}^{T}+\lambda_{R R}^{T}+\lambda_{R L}^{T}+\lambda_{L R}^{T}\right) c_{\theta}^{3}\right] \\
\text { where } \quad \mathcal{B}_{T}^{\text {int }}= & \mathcal{C}_{f}^{\text {int }{ }_{T}^{\mathrm{FC}}}\left(\frac{d_{\mathcal{U}}\left(d_{\mathcal{U}}-1\right)}{4}\right)\left[g_{s}^{2}\left(\frac{\hat{s}}{\Lambda_{\mathcal{U}}^{2}}\right)^{d_{\mathcal{U}}} A_{d_{\mathcal{U}}}\left[\cot \left(d_{\mathcal{U}} \pi\right)\right]\right]
\end{aligned}
$$

Here $\mathcal{C}_{f}^{\text {int }_{T}^{\mathrm{FC}}}=2$. Apart from an extra $\beta_{t}$ suppression in comparison to the vector unparticles, as seen from equation (32), the other salient differences between FC octet tensor and vector are:

1. Since the phase factor appearing in the tensor propagator is different to that of the vectors, the interference term contains an extra negative sign leading to a positive $A_{F B}^{t \bar{t}}$ for $n<d_{\mathcal{U}}<(n+1 / 2)$, zero for $d_{\mathcal{U}}=(n+1 / 2)$ and negative for $(n+1 / 2)<d_{\mathcal{U}}<$ $(n+1)$ interference with SM. Thus for FC tensor octet unparticle, $A_{F B}^{t \bar{t}}$ is positive for $1<d_{\mathcal{U}}<1.5$ and negative for $1.5<d_{\mathcal{U}}<2$ which is just opposite to that of FC vector unparticle as depicted in figure 11

2. Unlike FC vector the same helicity amplitudes also contribute to $A_{F B}^{t \bar{t}}$ for cases (a), (b) and (c) leading to $C_{F B}^{t \bar{t}} \neq A_{F B}^{t \bar{t}}$. The contribution to $A_{F B}^{t \bar{t}}$ from
$\left|\mathcal{M}_{\text {unp }}\right|^{2}$ is zero for cases (c) and (d). The same helicity and the opposite helicity contribution to $A_{F B}^{t \bar{t}}$ vanish for the interference term in case (d).

The opposite helicity contribution vanishes for case (d) (axial tensor) while it is non-zero for case (c). Thus while appreciable $A_{F B}^{t \bar{t}}$ contribution was coming in $\mathrm{FC}$ vector octet for axial vector couplings, the same is not true for FC tensor.

\section{B. Flavor Violating Vector Unparticle}

The unparticle theory being an effective theory it is possible to have flavor violating couplings of unparticles with quarks. The Flavor violating couplings involving first two generations of quarks and vector unparticles are tightly constrained. However, here we explore the vector unparticles which couples to the first and third gener- 


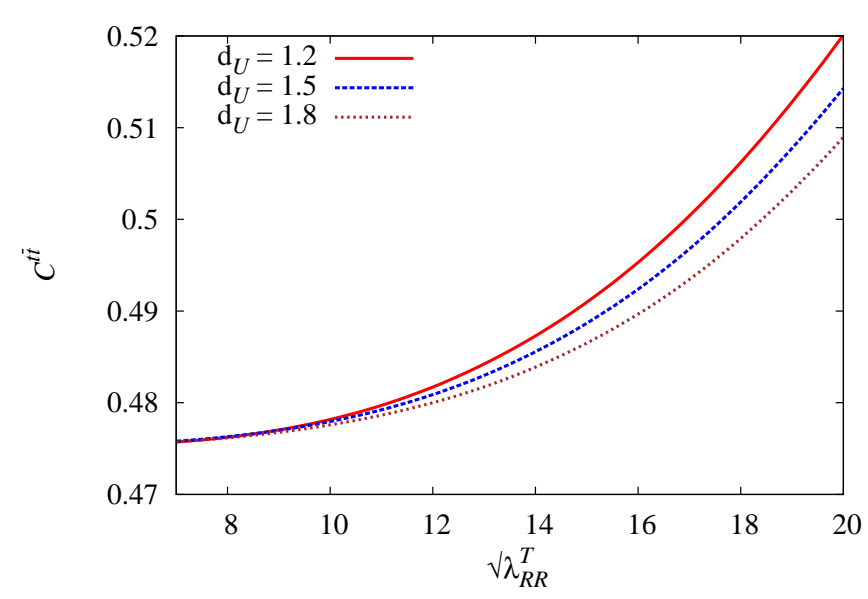

(a) $\lambda_{R R}^{T} \neq 0$ and $\lambda_{L R}^{T}=\lambda_{R L}^{T}=\lambda_{L L}^{T}=0$

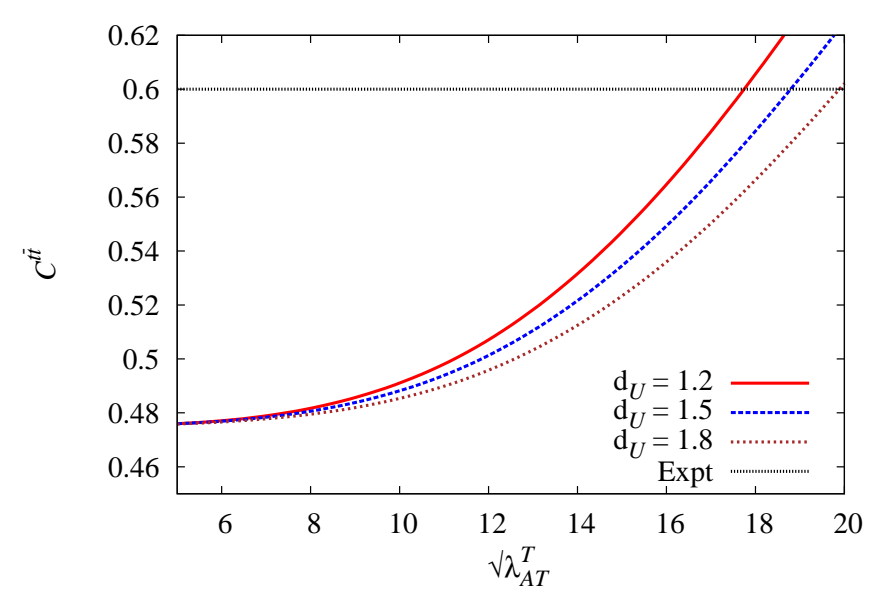

(c) $\lambda_{L L}^{T}=\lambda_{R R}^{T}=-\lambda_{R L}^{T}=-\lambda_{L R}^{T}=\lambda_{A T}$

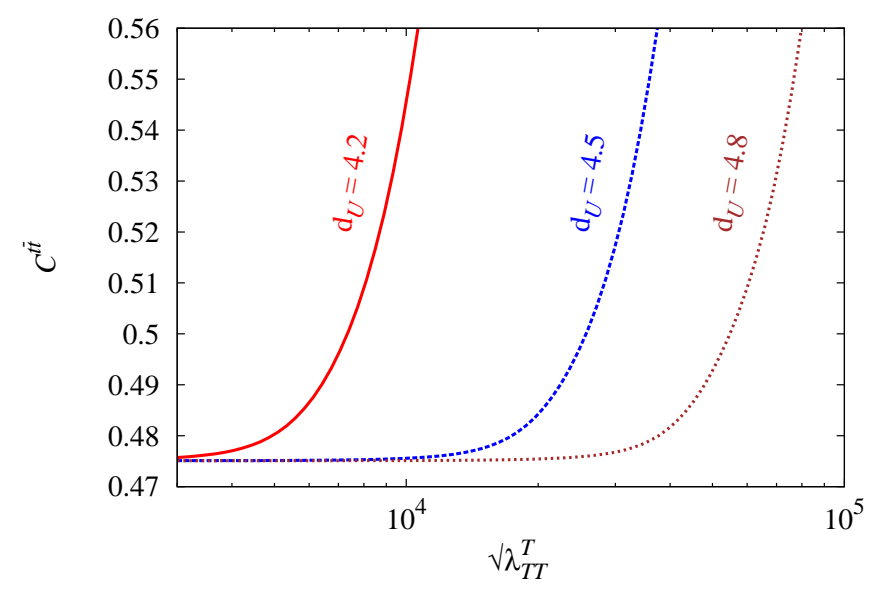

(e) $\lambda_{L L}^{T}=\lambda_{R R}^{T}=\lambda_{R L}^{T}=\lambda_{L R}^{T}=\lambda_{T T}^{T}$

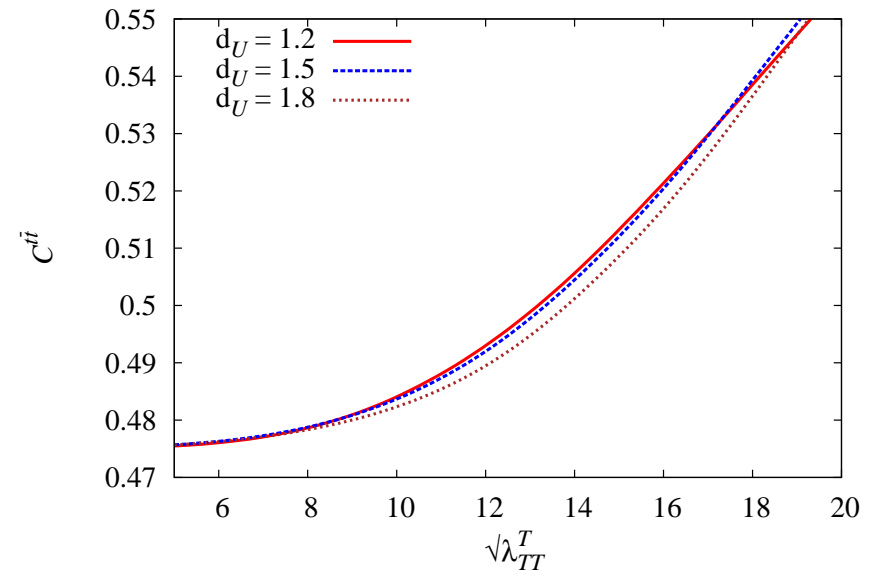

(b) $\lambda_{L L}^{T}=\lambda_{R R}^{T}=\lambda_{R L}^{T}=\lambda_{L R}^{T}=\lambda_{T T}^{T}$

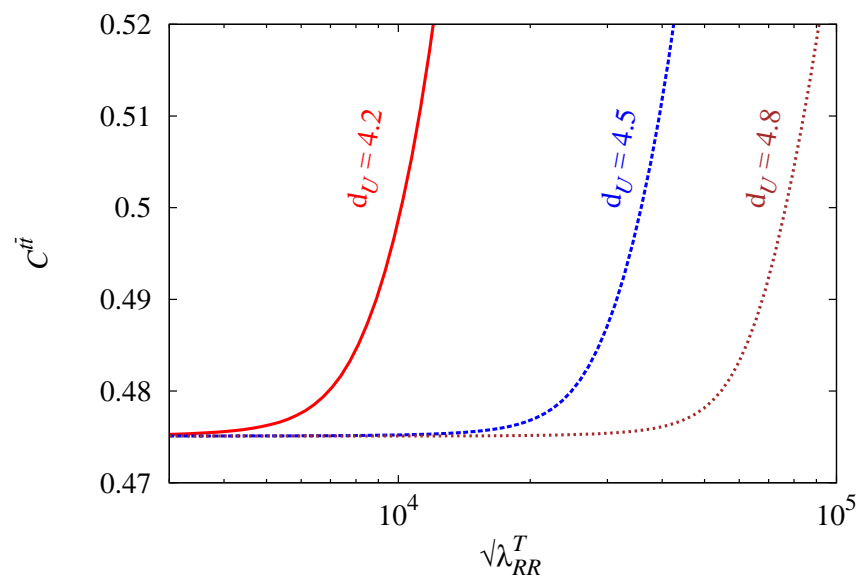

(d) $\lambda_{R R}^{T} \neq 0$ and $\lambda_{L R}^{T}=\lambda_{R L}^{T}=\lambda_{L L}^{T}=0$

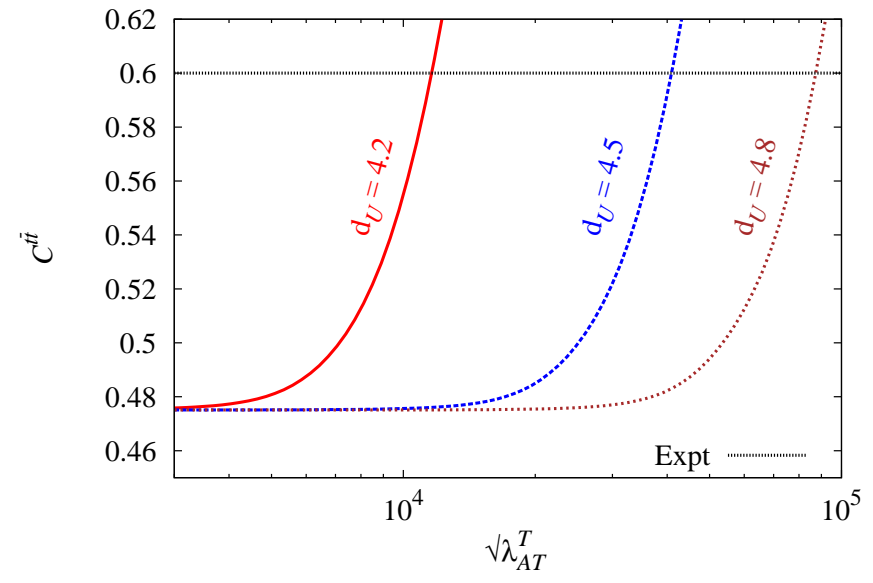

(f) $\lambda_{L L}^{T}=\lambda_{R R}^{T}=-\lambda_{R L}^{T}=-\lambda_{L R}^{T}=\lambda_{A T}$

FIG. 12: Variation of the spin-correlation coefficient $C^{t \bar{t}}$ evaluated in helicity basis with couplings $\sqrt{ } \lambda_{i j}^{T}$ for color octet flavor conserving tensor unparticles for various values of $d_{\mathcal{U}}$ at fixed $\Lambda_{\mathcal{U}}=1$ TeV and for different coupling combinations as mentioned in the text. The experimental value is depicted with a dot-dashed line at $0.60 \pm 0.50$ (stat) \pm 0.16 (syst) [28].

ation quarks only. The top pair production mediated by these unparticles are realized through $t$ channel pro- 


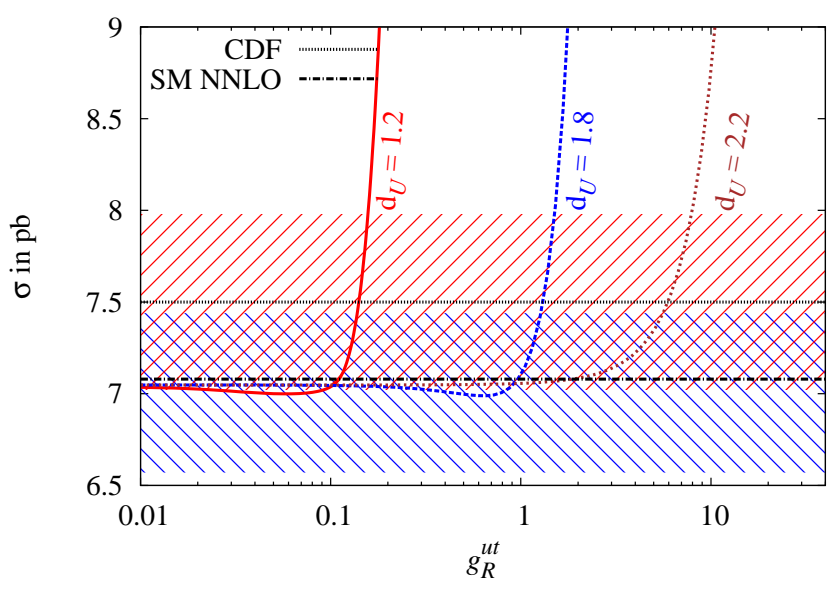

(a) $g_{L}^{u t}=0 \neq g_{R}^{u t}$

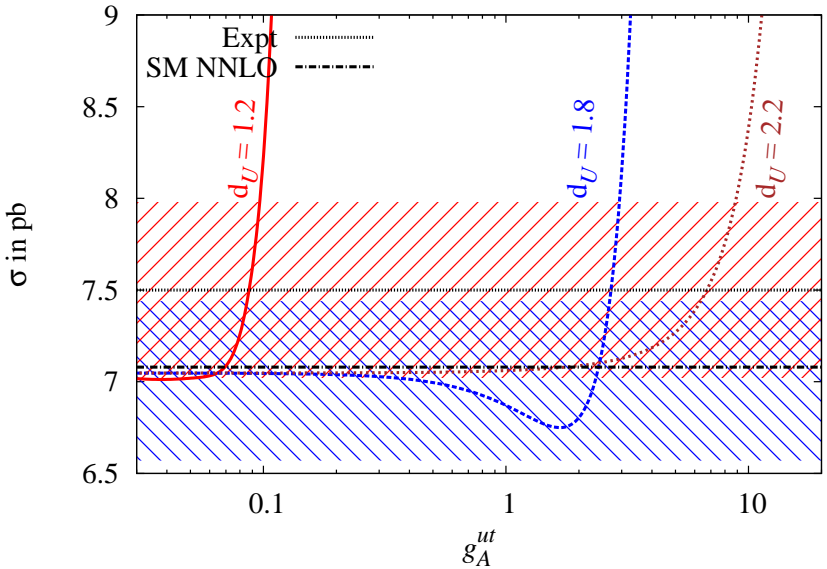

(b) $g_{R}^{u t}=-g_{L}^{u t}=g_{A}^{u t}$

FIG. 13: Variation of the cross-section $\sigma(p \bar{p} \rightarrow t \bar{t})$ with couplings $g_{i}^{u t}$ for color singlet flavor violating vector unparticles corresponding to different values of $d_{\mathcal{U}}$ in the range $1<d_{\mathcal{U}}<3$ at fixed $\Lambda_{\mathcal{U}}=1 \mathrm{TeV}$. The upper dotted line with a red band depicts the cross-section $7.50 \pm 0.48$ pb from CDF (all channels) [1], while the lower dot-dashed line with a blue band show theoretical estimate $7.08 \pm 0.36 \mathrm{pb}$ at NNLO [4] ]

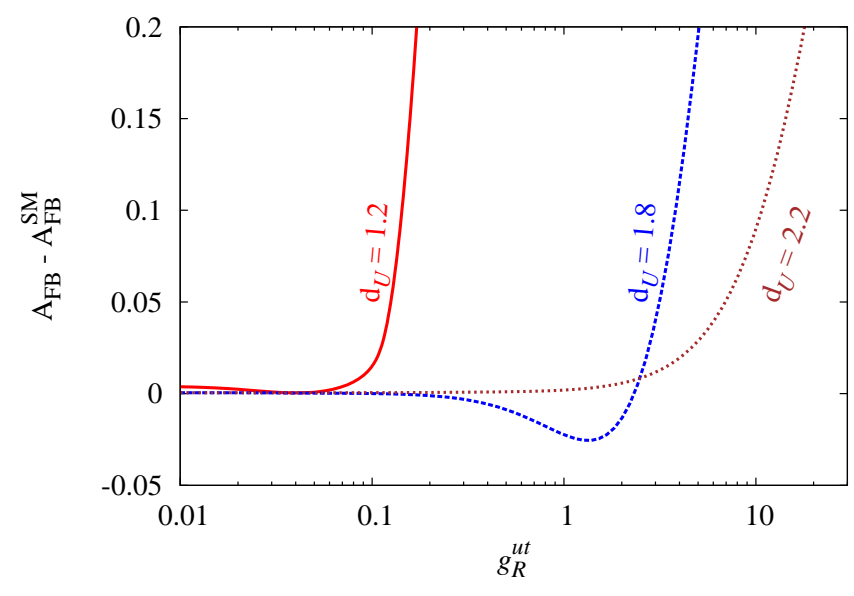

(a) $g_{L}^{u t}=0 \neq g_{R}^{u t}$

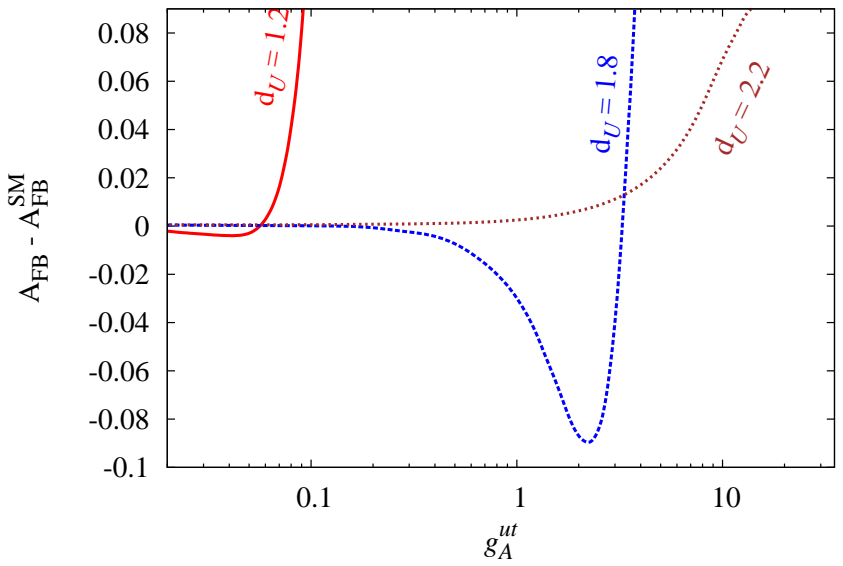

(b) $g_{R}^{u t}=-g_{L}^{u t}=g_{A}^{u t}$

FIG. 14: Variation of the unparticle contribution to charge asymmetry $A_{F B}-A_{F B}^{S M}$ with couplings $g_{i}^{u t}$ in the presence of flavor violating color singlet vector unparticles for various values of $d_{\mathcal{U}}$ in the range $1<d_{\mathcal{U}}<3$ at fixed $\Lambda_{\mathcal{U}}=1$ TeV. This is evaluated using the $1 d$ differential distribution of rapidity in $t \bar{t}$ rest frame.

cesses. Hence the second term in the propagator (19) also contributes.

It is to be noted that the vector unparticle propagator given in equation (16) has a pole for $d_{\mathcal{U}}=2$ at $\hat{t}=0$, and hence constraints the $d_{\mathcal{U}}$ to be greater than 2 [18, 50 for consistency of the theory. However in our case $|\hat{t}|$ is quite large and we do not encounter this problem. But this kind of flavor violating couplings also initiates the top decay to unparticles which is studied in section $\mathrm{V}$ This decay is only possible for $d_{\mathcal{U}}>2$. We can overcome this constraint by introducing a small infrared cut off which is discussed in section
VIB The implication of this broken scale invariance on the cross-section, $A_{F B}^{t \bar{t}}$ and spin-correlation is negligibly small as long as $\mu \ll|\hat{t}|$. This enables us to make our study for the top pair production in the region $d_{\mathcal{U}}>1$.

Following the notation introduced in the previous subsection, the four possible coupling for a given $d_{\mathcal{U}} \&$ $\Lambda_{\mathcal{U}}$ are (a) $g_{L}^{u t}=0 \neq g_{R}^{u t}$, (b) $g_{R}^{u t} \neq 0=g_{L}^{u t}$ (c) $g_{L}^{u t}=g_{R}^{u t}=g_{V}^{u t}$ and (d) $g_{L}^{u t}=-g_{R}^{u t}=g_{A}^{u t}$.

The $t$-channel process involving flavor violating singlet/octet unparticles interferes with QCD and negligibly small electroweak sector. Hence the results can be ex- 


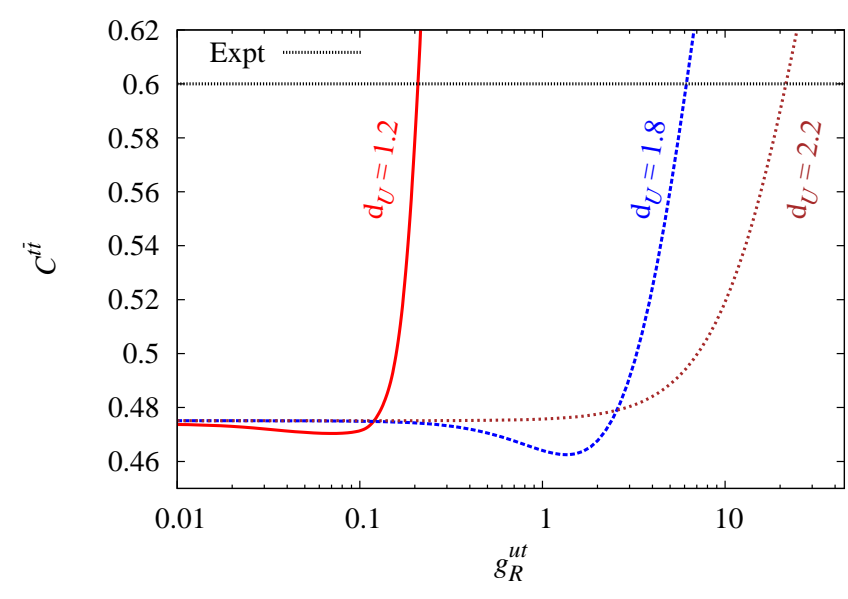

(a) $g_{L}^{u t}=0 \neq g_{R}^{u t}$

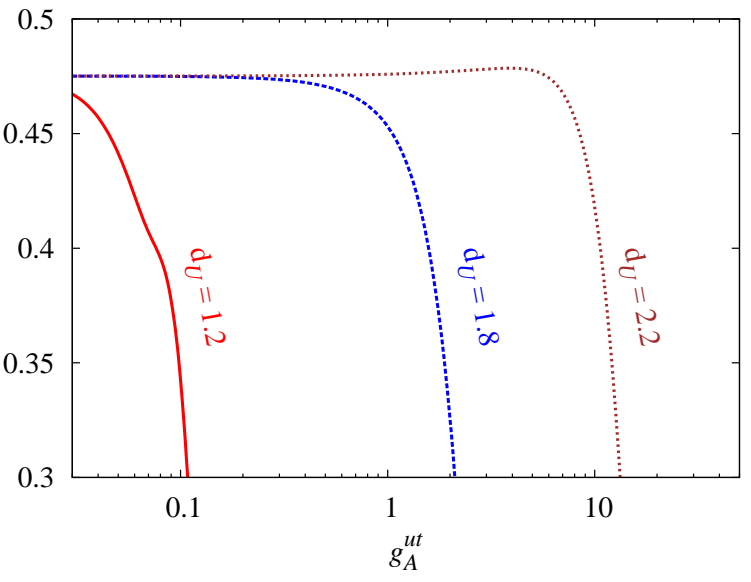

(b) $g_{R}^{u t}=-g_{L}^{u t}=g_{A}^{u t}$

FIG. 15: Variation of the spin correlation coefficient $C^{t \bar{t}}$ with couplings $g_{i}^{u t}$ in the presence of flavor violating color singlet vector unparticles for various values of $d_{\mathcal{U}}$ in the range $1<d_{\mathcal{U}}<3$ at fixed $\Lambda_{\mathcal{U}}=1$ TeV. The experimental value is depicted with a dot-dashed line at $0.60 \pm 0.50$ (stat) \pm 0.16 (syst) [28].

plained by the flavor violating unparticle new physics sector and its interference with QCD. Writing separately the same and opposite helicity contributions to these terms,

$$
\begin{aligned}
&\left|\mathcal{M}^{\text {unp }}\right|_{\text {same hel }}^{2}= c_{f}^{s q} g_{s}^{2} \mathcal{B}_{F V V}^{2}\left[\frac{1}{2}\left\{\left(g_{R}^{u t}\right)^{4}+\left(g_{L}^{u t}\right)^{4}\right\}\left(1-\beta_{t}^{2}\right)\left(1+A_{t}\right)^{2} s_{\theta}^{2}+4\left(g_{L}^{u t} g_{R}^{u t}\right)^{2} \times\right. \\
&\left.\left\{\left(1+\beta_{t}^{2}\right)\left(1+A_{t}^{2}\right)+\left(1+\beta_{t}^{2}\right) A_{t}^{2} c_{\theta}^{2}+4 \beta_{t} A_{t}+2\left(1+\beta_{t}^{2}+2 \beta_{t} A_{t}\right) A_{t} c_{\theta}\right\}\right](35) \\
&\left|\mathcal{M}^{\text {unp }}\right|_{\text {opp hel }}^{2}= c_{f}^{s q} g_{s}^{2} \mathcal{B}_{F V V}^{2}\left[4\left(g_{L}^{u t} g_{R}^{u t}\right)^{2}\left(1-\beta_{t}^{2}\right) A_{t}^{2} s_{\theta}^{2}+2\left\{\left(g_{R}^{u t}\right)^{4}+\left(g_{L}^{u t}\right)^{4}\right\} \times\right. \\
&\left.\left\{\left(1+A_{t}\right)^{2} \beta_{t} c_{\theta}+\frac{1}{4}\left(1+\beta_{t}^{2}\right)\left(1+A_{t}\right)^{2}\left(1+c_{\theta}^{2}\right)\right\}\right] \\
&\left.2 \mathcal{M}^{\mathrm{QCD}} \mathcal{R} e\left(\mathcal{M}^{\text {unp }}\right)\right|_{\text {same hel }}= c_{f}^{i n t} g_{s}^{2} \mathcal{B}_{\mathrm{FVV}}\left[\left\{\left(g_{L}^{u t}\right)^{2}+\left(g_{R}^{u t}\right)^{2}\right\}\left(1-\beta_{t}^{2}\right)\left(1+A_{t}\right) s_{\theta}^{2}\right] \\
&\left.2 \mathcal{M}^{\mathrm{QCD}} \mathcal{R} e\left(\mathcal{M}^{\mathrm{unp}}\right)\right|_{\text {opp hel }}= 2 c_{f}^{i n t} g_{s}^{2} \mathcal{B}_{\mathrm{FVV}}\left[\left\{\left(g_{L}^{u t}\right)^{2}+\left(g_{R}^{u t}\right)^{2}\right\}\left(1+A_{t}\right)\left(1+c_{\theta}^{2}+2 \beta_{t} c_{\theta}\right)\right] \\
& \mathcal{B}_{\mathrm{FVV}}=\left(\frac{1}{\Lambda_{\mathcal{U}}^{2}}\right)^{\left(d_{\mathcal{U}}-1\right)}\left[\frac{A_{d_{\mathcal{U}}}}{2 \sin \left(d_{\mathcal{U}} \pi\right)}\right](-\hat{t})^{\left(d_{\mathcal{U}}-2\right)} \hat{s} ; \quad A_{t}=\frac{a m_{t}^{2}}{4 \hat{t}} \text { and } a=\frac{2\left(d_{\mathcal{U}}-2\right)}{\left(d_{\mathcal{U}}-1\right)} \\
& \text { with }
\end{aligned}
$$

Here $c_{f}^{s q}=9(2)$ for FV singlet (octet) while $c_{f}^{\text {int }}=$ $4(-2 / 3)$ for FV singlet (octet).

The inherent symmetry of the helicity amplitudes makes the contribution to the observables identical for cases (a) and (b) and similarly for cases (c) and (d). The figures 13 16 give the variation of cross-section $\sigma^{t t}$, charge asymmetry $A_{F B}^{t \bar{t}}$ and spin correlation coefficient $C^{t \bar{t}}$ corresponding to the color singlet FV coupling, while the same variation for flavor violating color octet are given in figures 17 20. The salient features of these flavor violating unparticles are as follows:

1. Unlike the FC vector, the explicit and implicit de- 


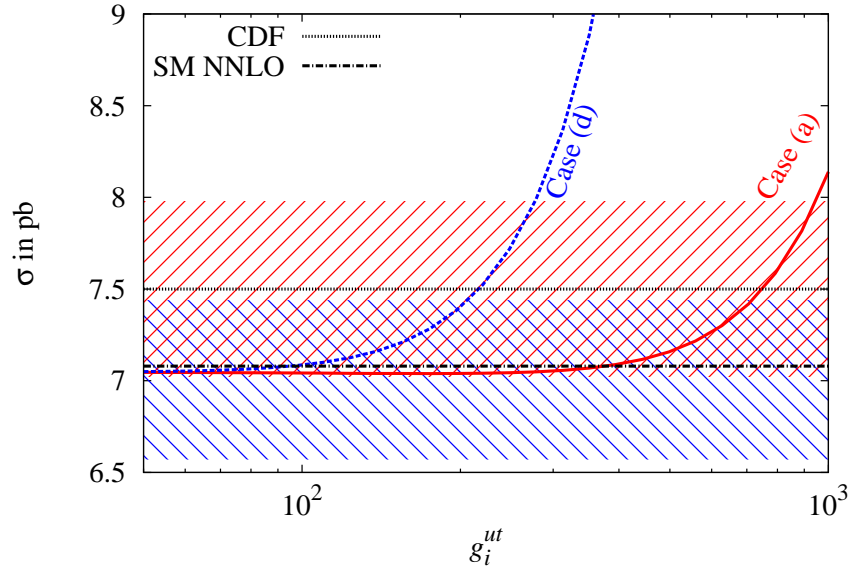

(a) $g_{i}^{u t}$ variation of $\sigma^{t \bar{t}}$ for cases (a) $g_{L}^{u t}=0 \neq g_{R}^{u t}$ and (d) $g_{R}^{u t}=-g_{L}^{u t}=g_{A}^{u t}$ with $d_{\mathcal{U}}=3.2$

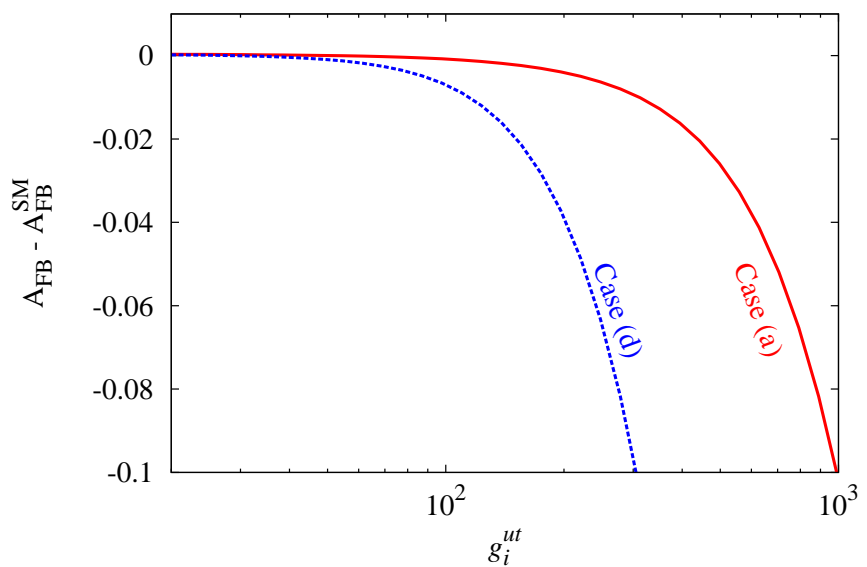

(b) $g_{i}^{u t}$ variation of $A_{F B}-A_{F B}^{S M}$ for cases (a) $g_{L}^{u t}=0 \neq g_{R}^{u t}$ and (d) $g_{R}^{u t}=-g_{L}^{u t}=g_{A}^{u t}$ with $d_{\mathcal{U}}=3.2$

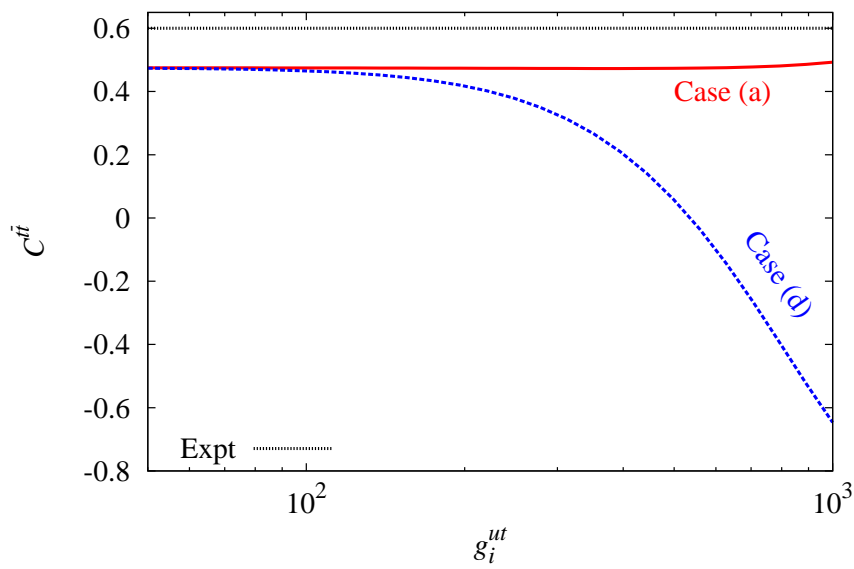

(c) $g^{u t}$ variation of $C^{t \bar{t}}$ for cases (a) and (d) with $d_{\mathcal{U}}=3.2$

FIG. 16: Variation of the cross-section $\sigma(p \bar{p} \rightarrow t \bar{t})$, the unparticle contribution to charge asymmetry $A_{F B}-A_{F B}^{S M}$ and spin correlation coefficient $C^{t \bar{t}}$ with couplings $g_{i}^{u t}$ for color singlet flavor violating vector unparticles at fixed $d_{\mathcal{U}}=3.2$ and $\Lambda_{\mathcal{U}}=1$ TeV corresponding to cases (a) and (d) mentioned in the text. In plot (a) the upper dotted line with a red band depicts the cross-section $7.50 \pm 0.48 \mathrm{pb}$ from CDF (all channels) [1], while the lower dot-dashed line with a blue band show theoretical

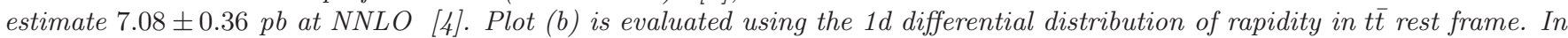
plot (c) the expertimental value is depicted with a dot-dashed line at $0.60 \pm 0.50$ (stat) \pm 0.16 (syst) [28].

pendence of $\hat{t}$ in the matrix element and in $A_{t}$ respectively restrains the straight forward simplification of the matrix element squared as a polynomial in $c_{\theta}$.

The contribution to $A_{F B}^{t \bar{t}}$ as well as $\sigma^{t \bar{t}}$ comes from both, same and opposite helicity amplitudes. Therefore $C_{F B}^{t}$ is not same as $A_{F B}^{t \bar{t}}$ when the flavor violating couplings are present.

2. $\hat{t}=m_{t}^{2}-(\hat{s} / 2)\left(1-\beta_{t} c_{\theta}\right)$ is always negative and $\sin \left(d_{\mathcal{U}} \pi\right)$ is negative for $(2 n-1)<d_{\mathcal{U}}<(2 n)$ while positive for $(2 n)<d_{\mathcal{U}}<(2 n+1)$. And finally the sign of color factor decide the sign of contribution of interference term of $\mathrm{FV}$ vector un- particle with QCD. Thus the interference term will be negative (positive ) for FV singlet (octet) for $1<d_{\mathcal{U}}<2$ while the sign will be reversed for $2<d_{\mathcal{U}}<3$. However the negative values for singlet are visible only for very small couplings as with large couplings $\left|\mathcal{M}^{\text {unp }}\right|^{2}$ contribution overshadows the interference term and hence the cross section, $A_{F B}^{t \bar{t}}$ and spin correlation for FV singlet first decrease then increase.

Since $-1<m_{t}^{2} /(4 \hat{t}) \leq 0$, it implies $\left|A_{t}\right| \leq 1$, the factor $\left(1+A_{t}\right)$ is always positive. Thus even though $a$ flips sign from positive for $1<d_{\mathcal{U}}<2$ to negative for $2<d_{\mathcal{U}}<3$, it does not affect the sign of 


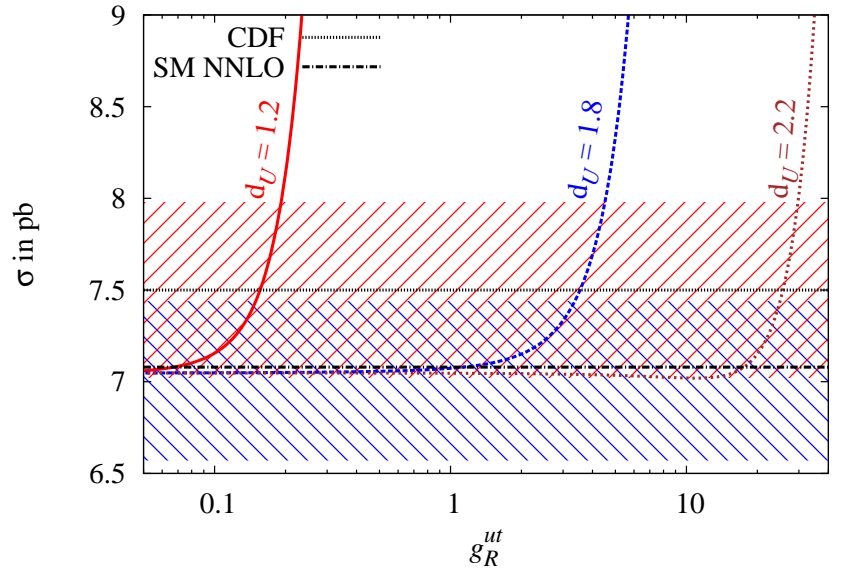

(a) $g_{L}^{u t}=0 \neq g_{R}^{u t}$

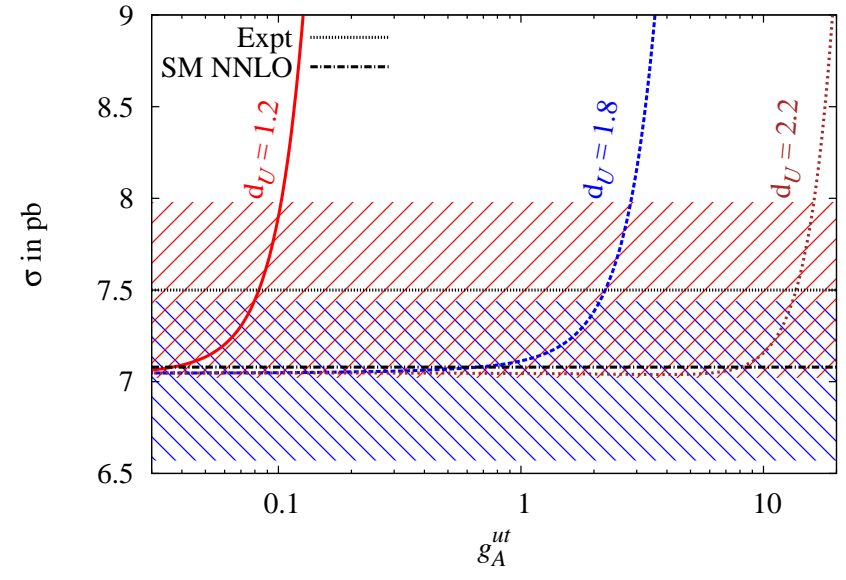

(b) $g_{R}^{u t}=-g_{L}^{u t}=g_{A}^{u t}$

FIG. 17: Variation of the cross-section $\sigma(p \bar{p} \rightarrow t \bar{t})$ with couplings $g_{i}^{u t}$ for color octet flavor violating vector unparticles for various values of $d_{\mathcal{U}}$ in the range $1<d_{\mathcal{U}}<3$ at fixed $\Lambda_{\mathcal{U}}=1$ TeV and for cases (a) (or (b)) and (d) (or (c)) mentioned in the text. The upper dotted line with a red band depicts the cross-section $7.50 \pm 0.48$ pb from CDF (all channels) [1], while the lower dot-dashed line with a blue band show theoretical estimate $7.08 \pm 0.36 \mathrm{pb}$ at NNLO [4]].

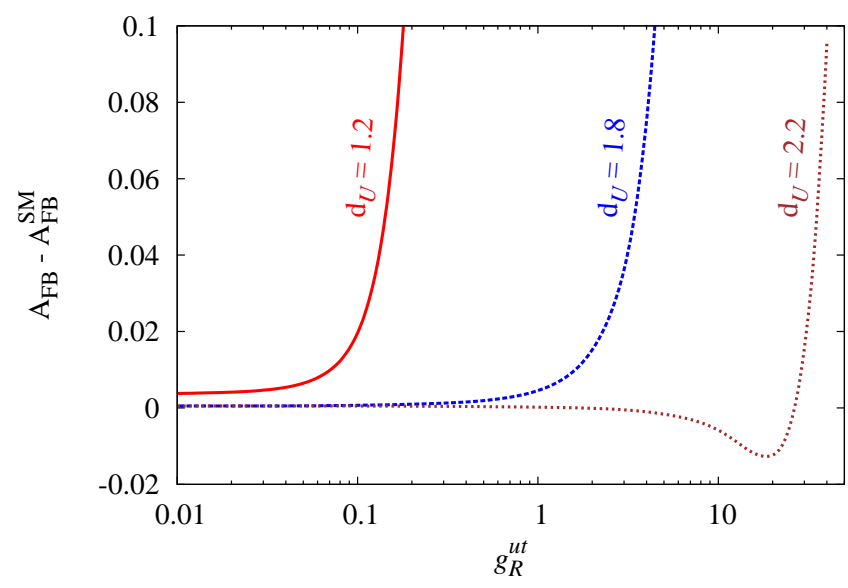

(a) $g_{L}^{u t}=0 \neq g_{R}^{u t}$

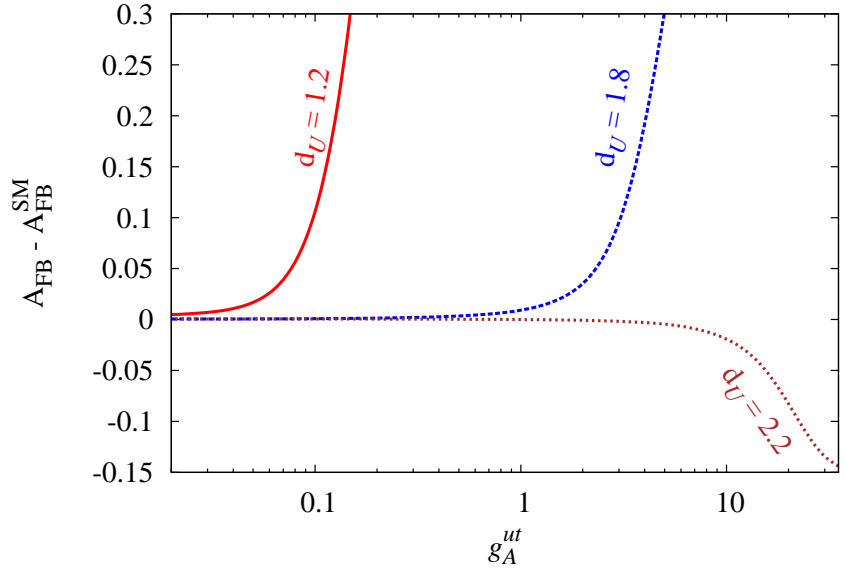

(b) $g_{R}^{u t}=-g_{L}^{u t}=g_{A}^{u t}$

FIG. 18: Variation of the unparticle contribution to charge asymmetry $A_{F B}^{S M}$ with couplings $g_{i}^{\text {ut }}$ in the presence of flavor violating color octet vector unparticles for various values of $d_{\mathcal{U}}$ in the range $1<d_{\mathcal{U}}<3$ at fixed $\Lambda_{\mathcal{U}}=1$ TeV and for the cases

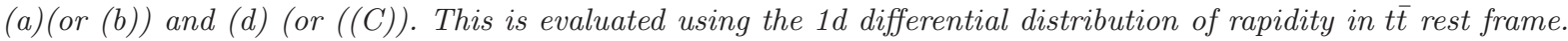

interference term for the $d_{\mathcal{U}}$ values considered.

Thus for coupling to be within the range that gives $\sigma^{t \bar{t}}$ consistent with the CDF value, FV singlet vector unparticle gives favorable $A_{F B}^{t \bar{t}}$ only for $d_{\mathcal{U}}$ close to 1 . On the other hand octet gives favorable values for the entire range $1<d_{\mathcal{U}}<2$. For $2<d_{\mathcal{U}}<3$, octet gives negative $A_{F B}^{t \bar{t}}$ for allowed values of couplings while singlet gives very low values.

\section{FV COUPLINGS: SAME SIGN TOPS AND TOP DECAY WIDTH}

As we have introduced the FV neutral current via non diagonal coupling for the fermion-fermion unparticle involving first and third generation, it is likely to have its bearings on the same sign top quark production and top decay width [51]. 


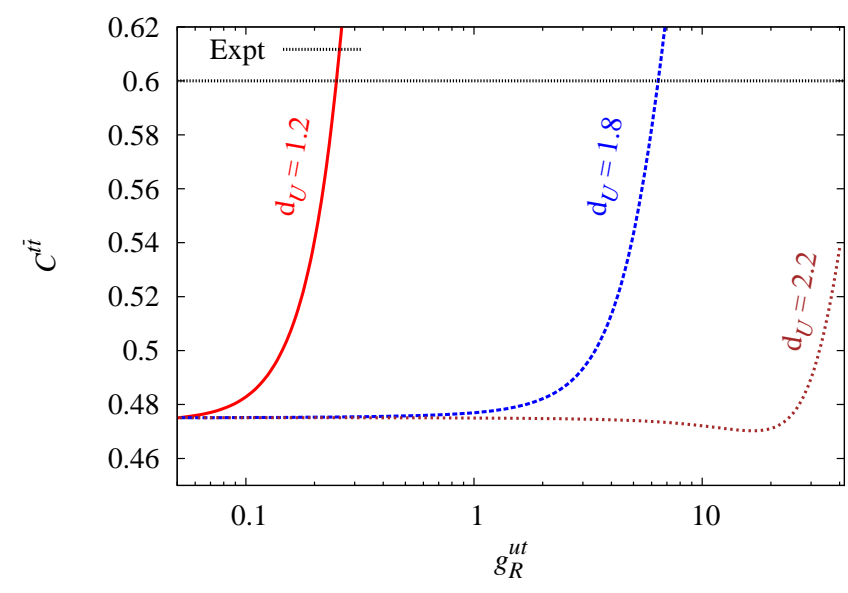

(a) $g_{L}^{u t}=0 \neq g_{R}^{u t}$

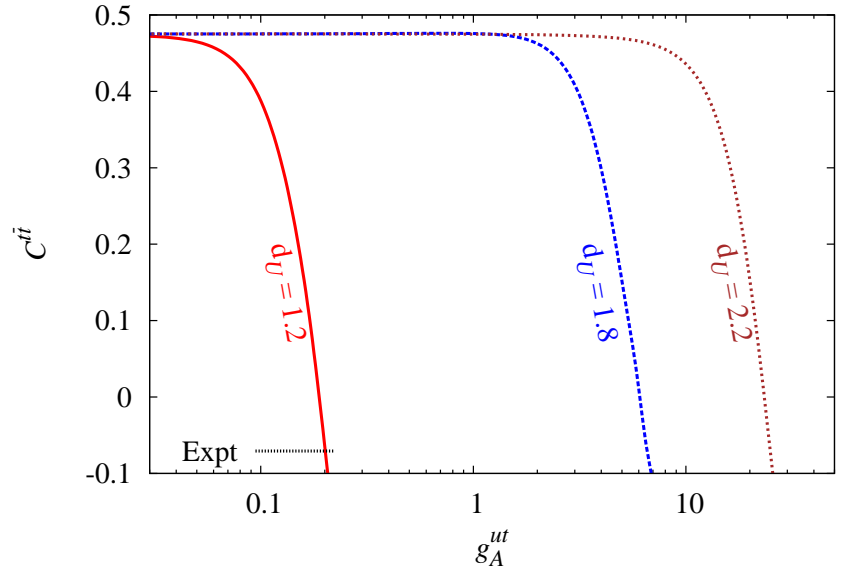

(b) $g_{R}^{u t}=-g_{L}^{u t}=g_{A}^{u t}$

FIG. 19: Variation of the spin correlation coefficient $C^{t \bar{t}}$ with couplings $g_{i}^{\text {ut }}$ in the presence of flavor violating color octet vector unparticles for various values of $d_{\mathcal{U}}$ in the range $1<d_{\mathcal{U}}<3$ at fixed $\Lambda_{\mathcal{U}}=1$ TeV and for cases (a)(or(b)) and (d)(or (c)) mentioned in the text. The experimental value is depicted with a dot-dashed line at $0.60 \pm 0.50$ (stat) \pm 0.16 (syst) [28].

\section{A. Same Sign Top Production}

Recently an inclusive search of same sign tops at CMS dis-favored the FCNC solution in $Z^{\prime}$ model for $A_{F B}^{t \bar{t}}$ anomaly at TeVatron [52]. The SM contribution is highly suppressed and any affirmative signal would indicate a presence of new physics. The unparticle theory through their FV couplings also generates the same sign tops $p \bar{p} \rightarrow t t$ via $t$ and $u$ channel Feynman diagrams. We compute the parton level helicity amplitudes for $u u \rightarrow t t$ and are given in the Appendix A 5

As mentioned earlier, we have performed our analysis with $\Lambda_{\mathcal{U}}=1 \mathrm{TeV}$ to probe the physics at TeVatron, where the partonic c.m. energy $<<1 \mathrm{TeV}$. However, to examine the physics at LHC and validate the same model, we need to enhance this scale at least to $\Lambda_{\mathcal{U}}=10$ $\mathrm{TeV}$. In this light at present we only see the effect of the unparticle physics at TeVatron and check their crossvalidity among various processes. Same sign top production was also constrained by TeVatron 53. The CDF data was based on same sign dilepton search and their observations are summarized in the table II of reference [53]. They predicted the upper limits at 95\% CL, on the production cross-section $\sigma(p \bar{p} \rightarrow t t+\overline{t t})$ times branching ratio $\operatorname{BR}(W \rightarrow l \nu)^{2}$ for all distinct chirality modes: leftleft $(L L)$ to be $54 \mathrm{fb}$, right-right $(R R)$ to be $51 \mathrm{fb}$ and left-right $(L R)$ to be $51 \mathrm{fb}$, assuming only one non-zero mode at one time. We present the $95 \%$ CL contours for varying $d_{\mathcal{U}}$ on $\left|g_{L}^{u t}\right|-\left|g_{R}^{u t}\right|$ plane using the result from $L L$ mode, assuming that there is remarkable difference in efficiency or shape among the contribution from $L L, L R$ or $R R$ modes. The contours corresponding to color singlets and octets are shown in figures 21a and 21b respectively.

We expect that with the change of the scale $\Lambda_{\mathcal{U}}$, we will be able to probe the same parameter region for LHC. This work is currently under progress.

\section{B. Top Decay Width}

The total decay width of top quark is one of the fundamental property of top physics. It is measured with precision from the partial decay width $\Gamma(t \rightarrow W b)$ in the $t$ channel of the single top quark production and from $t \bar{t}$ events. Recently the top total width is measured to be $\Gamma_{t}=1.99_{-0.55}^{+0.69} \mathrm{GeV}$ corresponding to $2.3 \mathrm{fb}^{-1}$ data by $\mathrm{D} \varnothing$ collaboration [54]. The SM contribution to top decay width at NLO in $\alpha_{s}$ is given as

$$
\Gamma_{S M}(t \rightarrow W b)=\frac{G_{F} m_{t}^{3}}{8 \pi \sqrt{2}}\left|V_{t b}\right|^{2}\left(1-\frac{M_{W}^{2}}{m_{t}^{2}}\right)^{2}\left(1+2 \frac{M_{W}^{2}}{m_{t}^{2}}\right)\left[1-\frac{2 \alpha_{s}}{3 \pi}\left(\frac{2 \pi^{2}}{3}-\frac{5}{2}\right)\right]
$$

Computing with $\alpha_{s}\left(M_{Z}\right)=0.118, G_{F}=1.16637 \times 10^{-5}$ $\mathrm{GeV}^{-2}, M_{W}=80.399 \mathrm{GeV},\left|V_{t b}\right|=1$, and $m_{t}=173$
$\mathrm{GeV}$, we find $\Gamma_{S M}=(t \rightarrow W b)_{S M}=1.34 \mathrm{GeV}$. 


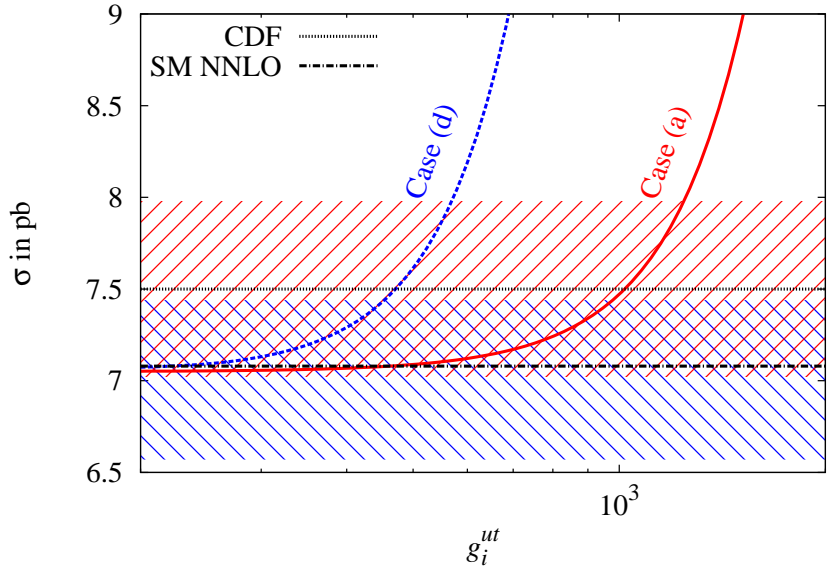

(a) $g_{i}^{u t}$ variation of $\sigma^{t \bar{t}}$ for cases (a) $g_{L}^{u t}=0 \neq g_{R}^{u t}$ and (d) $g_{R}^{u t}=-g_{L}^{u t}=g_{A}^{u t}$ with $d_{\mathcal{U}}=3.2$

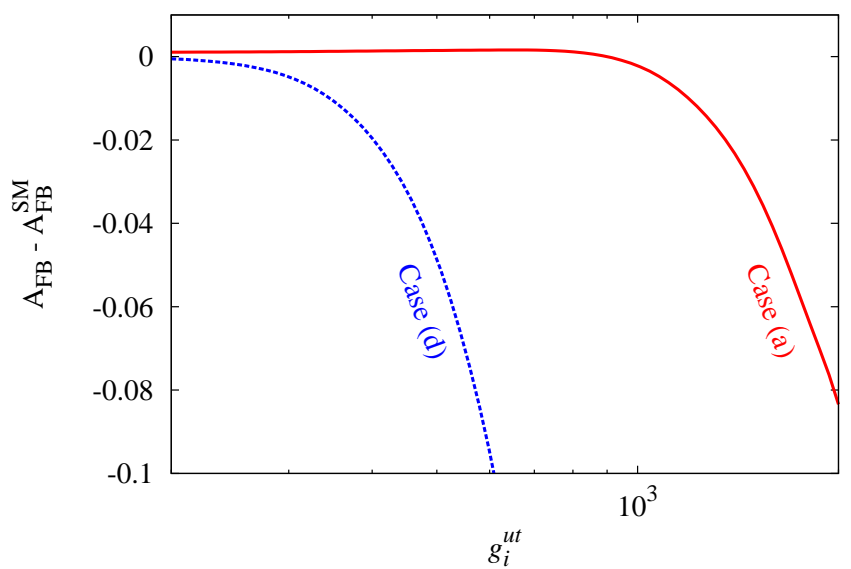

(b) $g_{i}^{u t}$ variation of $A_{F B}-A_{F B}^{S M}$ for cases (a) $g_{L}^{u t}=0 \neq g_{R}^{u t}$ and (d) $g_{R}^{u t}=-g_{L}^{u t}=g_{A}^{u t}$ with $d_{\mathcal{U}}=3.2$

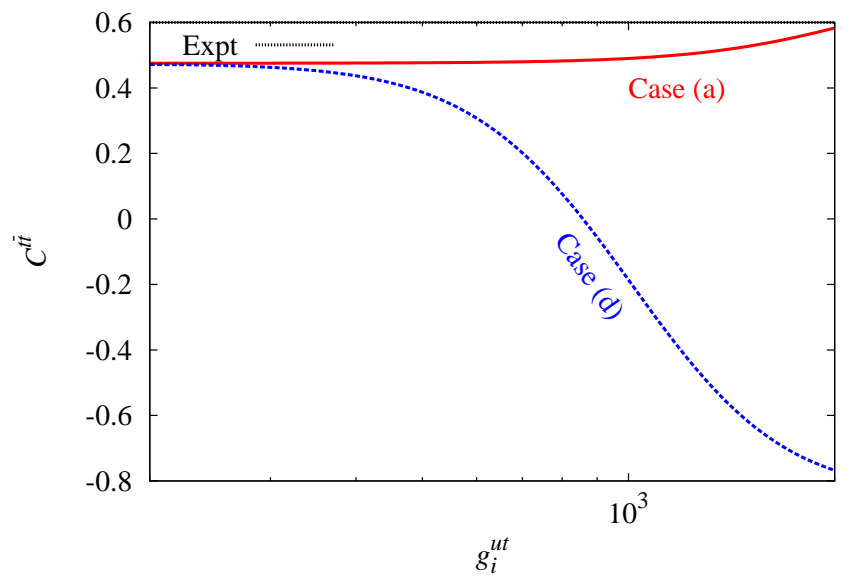

(c) $g_{i}^{u t}$ variation of $C^{t \bar{t}}$ for cases (a) $g_{L}^{u t}=0 \neq g_{R}^{u t}$ and (d)

$$
g_{R}^{u t}=-g_{L}^{u t}=g_{A}^{u t} \text { with } d_{\mathcal{U}}=3.2
$$

FIG. 20: Variation of the cross-section $\sigma(p \bar{p} \rightarrow t \bar{t})$, the unparticle contribution to charge asymmetry $A_{F B}-A_{F B}^{S M}$ and the spin correlation coefficient $C^{t \bar{t}}$ with couplings $g_{i}^{u t}$ for color octet flavor violating vector unparticles at fixed $d_{\mathcal{U}}=3.2$ and $\Lambda_{\mathcal{U}}=1$ TeV corresponding to cases (a) and (d) mentioned in the text. In plot (a) the upper dotted line with a red band depicts the cross-section $7.50 \pm 0.48 \mathrm{pb}$ from CDF (all channels) [1], while the lower dot-dashed line with a blue band show theoretical estimate $7.08 \pm 0.36 \mathrm{pb}$ at $N N L O$ [4]. Plot (b) is evaluated using the $1 d$ differential distribution of rapidity in t $\bar{t}$ rest frame. In plot (c) the experimental value is depicted with a dot-dashed line at $0.60 \pm 0.50$ (stat) \pm 0.16 (syst) [28].

In unparticle sector, this was first mooted by Georgi [17] where he considered the flavor violating derivative coupling of the scalar unparticles given as $i \lambda \Lambda^{-d_{\mathcal{U}}} \bar{u} \gamma_{\mu}\left(1-\gamma_{5}\right) t \partial^{\mu} \mathcal{O}_{\mathcal{U}}+$ h.c. . The FV vector unparticle initiates a new channel for top decaying to un- particle and a lighter quark $q$. We compute the partial decay width $\Gamma_{\mathcal{U}}\left(t \rightarrow q \mathcal{U}^{V}\right)$ as given in the Appendix B. This decay width diverges for $d_{\mathcal{U}}<2$ while for $d_{\mathcal{U}}>2$ it is given as

$$
\Gamma_{t}=\Gamma_{S M}+\frac{N_{C}}{6} \frac{A_{d_{\mathcal{U}}}}{4 \pi^{2}} g_{s}^{2}\left[\left(g_{L}^{\mathcal{U}_{V}^{\mathrm{n}} t q}\right)^{2}+\left(g_{R}^{\mathcal{U}_{V}^{\mathrm{n}} t q}\right)^{2}\right]\left(\frac{m_{t}^{2}}{\Lambda_{\mathcal{U}}^{2}}\right)^{d_{\mathcal{U}}-1} \frac{m_{t}}{4}\left[\frac{a-4+(a+2) d_{\mathcal{U}}}{\left(d_{\mathcal{U}}-2\right)\left(d_{\mathcal{U}}-1\right) d_{\mathcal{U}}\left(d_{\mathcal{U}}+1\right)}\right]
$$

However if the scale invariance is broken at a scale $\mu \neq 0$, one can evaluate the decay width in the region
$1<d_{\mathcal{U}}<2$. As mentioned earlier inclusion of such mass 


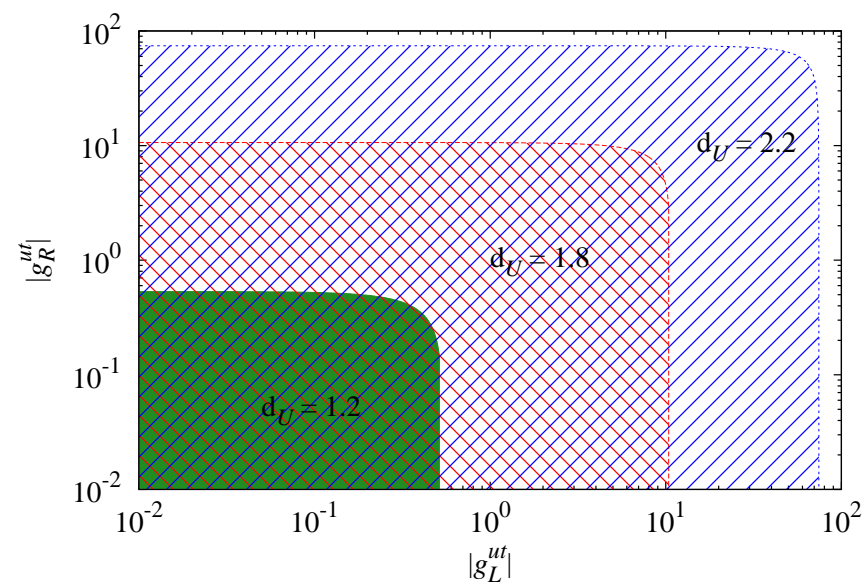

(a) Flavor Violating Singlet Vector Unparticle

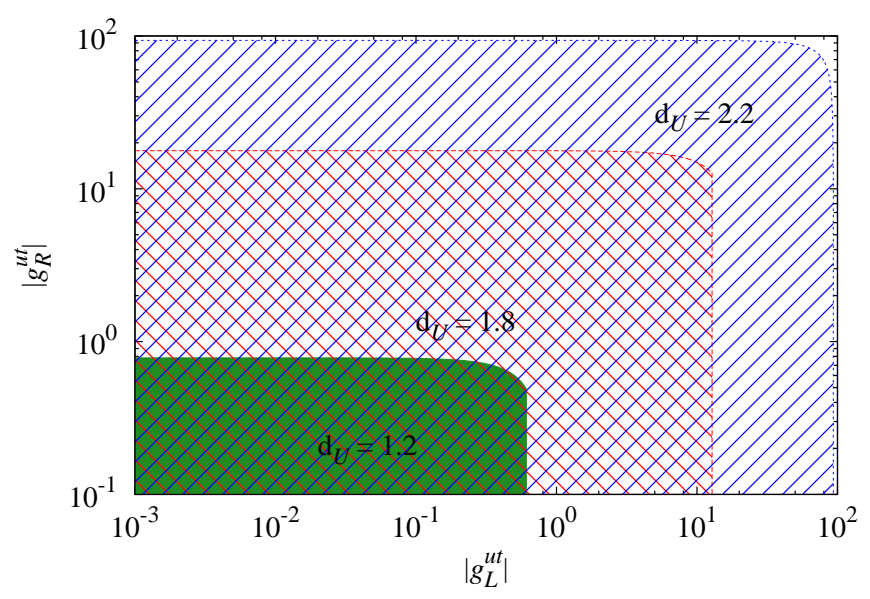

(b) Flavor Violating Octet Vector Unparticle

FIG. 21: Exclusion contours at 95\% CL corresponding to the cross-section $\sigma(p \bar{p} \rightarrow t t+\overline{t t})=54 f b[52]$ on the $\left|g_{L}^{u t}\right|-\left|g_{R}^{u t}\right|$ plane for a given $d_{\mathcal{U}}$ and $\Lambda_{\mathcal{U}}=1 \mathrm{TeV}$. The value of the couplings bounded by the enclosed region (for a specific du value) are allowed.

gap also protects the theory from realizing continuous spectrum of unparticles through the decay process. Introduction of such a scale however does not modify the cross sections for $\mu \ll m_{t \bar{t}}$. We depict the exclusion contours with various choices of the breaking scales at $1 \mathrm{GeV}, 10$ $\mathrm{GeV}$ and at $m_{Z}=91.18 \mathrm{GeV}$ corresponding to the color octet unparticles on $\left|g_{L}^{u t}\right|-\left|g_{R}^{u t}\right|$ plane. These contours show the allowed range of these couplings constrained from the observed total top decay width $\Gamma_{t}=\Gamma_{S M}+\Gamma_{\mathcal{U}}$ at $\mathrm{D} \varnothing$. The nature and behaviour of the contribution from the color singlet flavor violating unparticles are similar to that of the octet. Therefore we do not provide the corresponding contours separately. We find from figure 22 that the parameter region which contributes to $t \bar{t}$ events considerably along with large positive $A_{F B}^{t \bar{t}}$, shrinks and hence is much more constrained. We also observe that the increase in the scale invariance breaking scale relaxes the bound on the couplings.

\section{ANALYSIS AND SUMMARY}

\section{A. $m_{t \bar{t}}$ distribution of $A_{F B}^{t \bar{t}}$}

We scan our parameters and perform $\chi^{2}$ analysis for both FC and FV cases and predict the set of best parameters which can possibly explain the $A_{F B}^{t \bar{t}}$ anomaly. To perform this analysis we take into account the $A_{F B}^{t \bar{t}}$ distribution over $m_{t \bar{t}}$ bins from the full Run II TeVatron Dataset [7]. We define the $\chi^{2}$ as

$$
\chi^{2}=\sum_{i=1}^{n} \frac{\left(\mathcal{O}_{i}^{t h}-\mathcal{O}_{i}^{e x p}\right)^{2}}{\left(\delta \mathcal{O}_{i}^{e x p}\right)^{2}}
$$

where $i$ is $m_{t \bar{t}}$ bin index, $\mathcal{O}_{i}^{t h}, \mathcal{O}_{i}^{\exp }$ and $\delta \mathcal{O}_{i}^{\text {exp }}$ are the $\mathrm{SM}+$ model estimate, experimental measurement and its error in the corresponding $i^{\text {th }}$ bin respectively. Following the reference [7], we quote and use the experimental data based on the complete dataset of Run II at an integrated luminosity $8.7 \mathrm{fb}^{-1}$ ( given in the table II) to compute $\chi_{\min }^{2}$. The first column reads the bin size while the second and third column of the table $\amalg$ gives experimentally observed value of $A_{F B}^{t \bar{t}}$ in each of these bins with its error and the expected number from NLO (QCD+EW) with backgrounds respectively. We also add the observed cross-section as an eighth observable in equation (41) $\sigma(p \bar{p} \rightarrow t \bar{t})=7.5 \pm 0.31$ (stat) \pm 0.34 (syst) \pm 0.15 (Z theory) $\mathrm{pb}[1]$. The two dimensional parameter space $\left(\left(\sqrt{\lambda_{L L}}\right.\right.$, $\left.\sqrt{\lambda_{R R}}\right)$ for FC cases and $\left(g_{L}^{u t}, g_{R}^{u t}\right)$ for the FV case) for fixed $d_{\mathcal{U}}$ and $\Lambda_{\mathcal{U}}$ is scanned leading to the minimum value of the $\chi^{2} \equiv \chi_{\min }^{2}$.

At $\chi_{\min }^{2}$ the corresponding parameter points are likely to be the best possible model parameters in the unparticle physics at $\Lambda_{\mathcal{U}}=1 \mathrm{TeV}$, which are consistent with all the observations. Fourth-seventh columns in the table exhibit the $m_{t \bar{t}}$ spectrum of $A_{F B}^{t \bar{t}}$ corresponding to the four distinct cases of best fitted model parameters. In the figure 23, we plot histograms showing the $m_{t \bar{t}}$ spectrum of $A_{F B}^{t \bar{t}}$ for all the four cases. We have shown and compared the slope of our best-fit line with that from the experimental data in this figure and table I.

\section{B. Effect of Mass gap}

In our study we have not introduced any infra-red cutoff in the theory of unparticles modulo top decay width. The existence of massless fields in a theory gives rise to severe modification in the low energy phenomenology which 


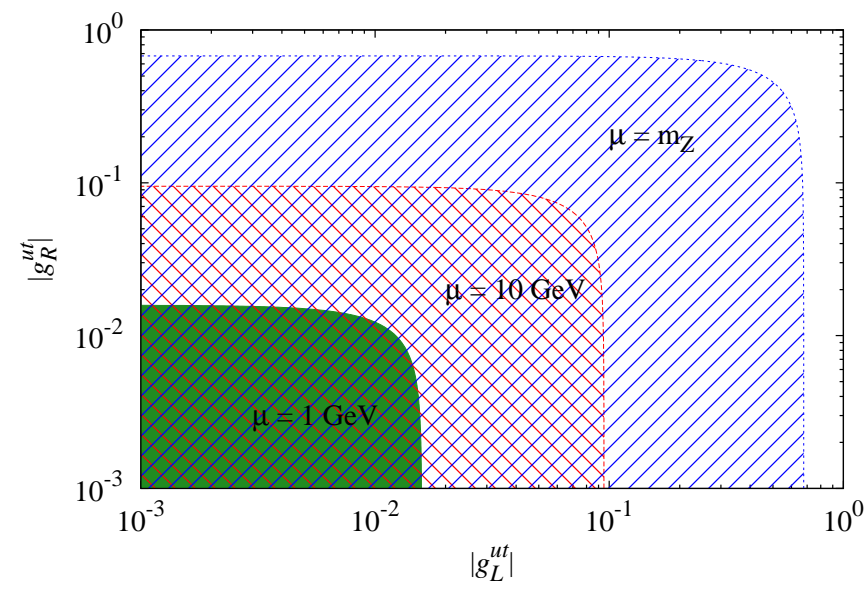

(a) $d_{\mathcal{U}}=1.2$

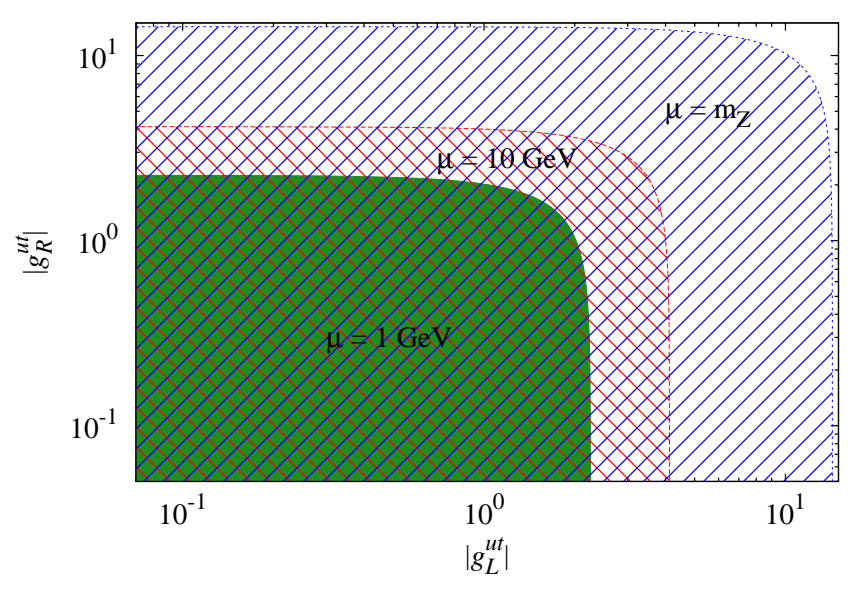

(b) $d_{\mathcal{U}}=1.8$

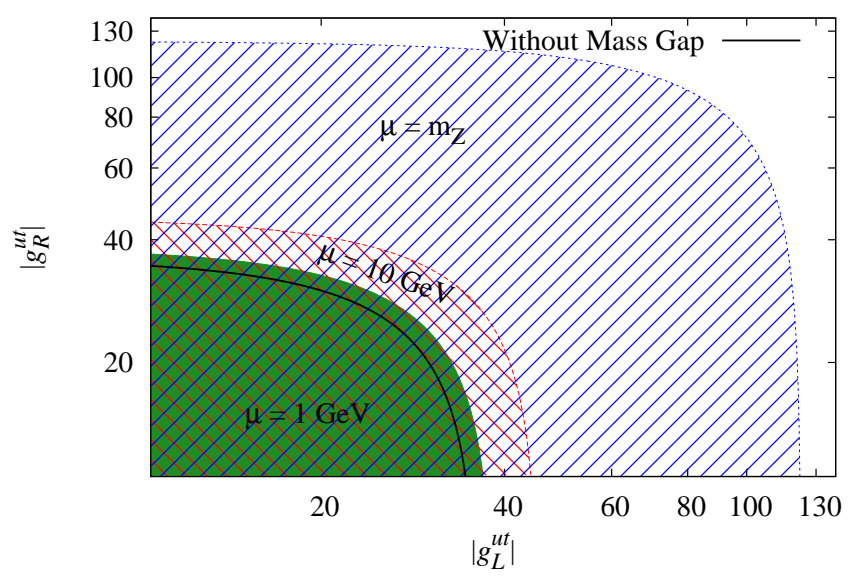

(c) $d_{\mathcal{U}}=2.2$

FIG. 22: Exclusion contours at 95\% CL corresponding $\Gamma_{t}=1.99_{-0.55}^{+0.69}$ GeV [54] on the $\left|g_{L}^{u t}\right|-\left|g_{R}^{u t}\right|$ plane for flavor Violating octet vector Unparticle with a given mass gap $\mu$ and $\Lambda_{\mathcal{U}}=1 \mathrm{TeV}$. The value of the couplings bounded by the enclosed region (for a specific mass $\mu$ value) are allowed. In figure (c) the black contour line corresponds to $\mu=0 \mathrm{GeV}$.

is successfully explained by SM alone. To be precise, if there is no cut-off in the theory of unparticles there would be massive production of color singlet/ octet unparticles in colliders.

A standard way to treat the breaking of conformal symmetry is to introduce a mass gap in the spectral density and thereby removing modes with energy less than the infrared cutoff scale $\mu$ in the spectral density. Thus the propagator for vector unparticle given in equation (19) will be modified to

$$
\Delta^{\mu \nu}(p)=\frac{-i A_{d_{\mathcal{U}}}}{2 \sin \left(d_{\mathcal{U}} \pi\right)}\left[-\left(p^{2}-\mu^{2}\right)\right]^{d_{\mathcal{U}}-2}\left(-g^{\mu \nu}+a \frac{p^{\mu} p^{\nu}}{p^{2}}\right)
$$

with

$$
\left[\left(-\left(p^{2}-\mu^{2}\right)\right]^{d_{\mathcal{U}}-2}= \begin{cases}\left|p^{2}-\mu^{2}\right|^{d_{\mathcal{U}}-2} & \text { for } p^{2}<\mu^{2} \\ \left|p^{2}-\mu^{2}\right|^{d_{\mathcal{U}}-2} e^{i d_{\mathcal{U}} \pi} & \text { for } p^{2}>\mu^{2}\end{cases}\right.
$$

It reduces to (19) in the limit $\mu \rightarrow 0$. From equa-

tion (43) it follows that one can ignore the existence 


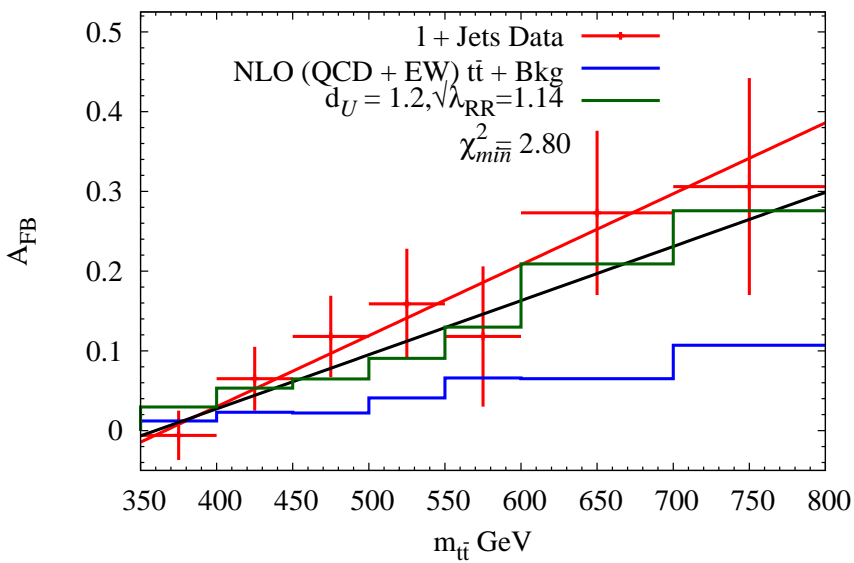

(a) FC Vector Singlet: $\lambda_{R R} \neq 0$ and $\lambda_{L R}=\lambda_{R L}=\lambda_{L L}=0$

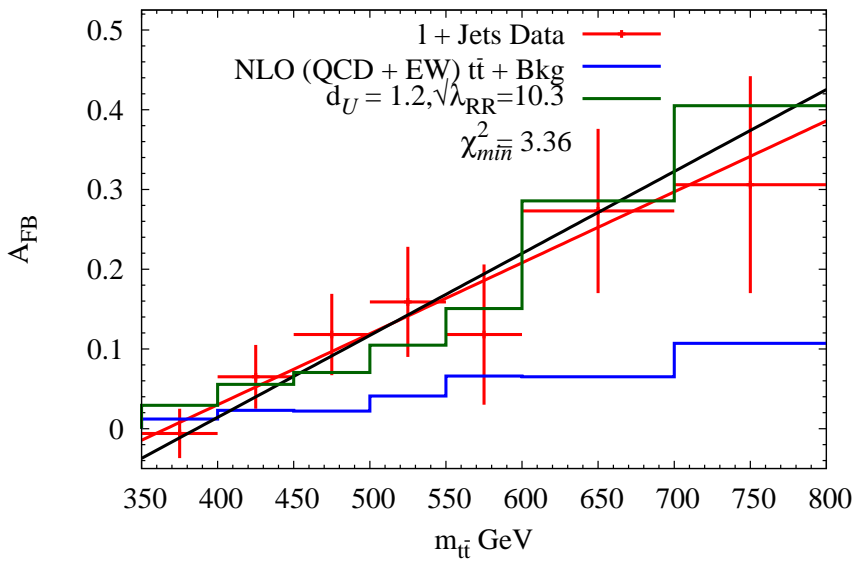

(c) FC Tensor Octet: $\lambda_{L L}=\lambda_{R R}=-\lambda_{R L}=-\lambda_{L R}=\lambda_{A A}$

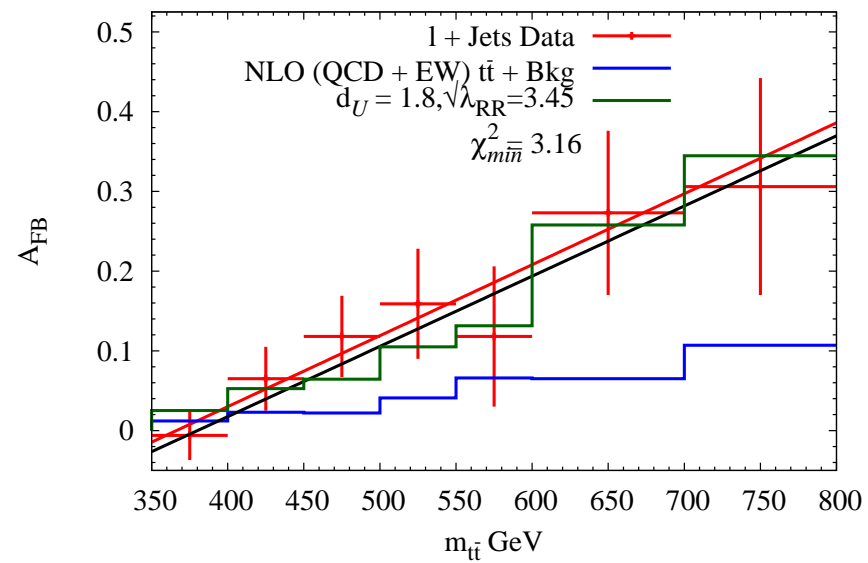

(b) FC Vector Octet: $\lambda_{R R} \neq 0$ and $\lambda_{L R}=\lambda_{R L}=\lambda_{L L}=0$

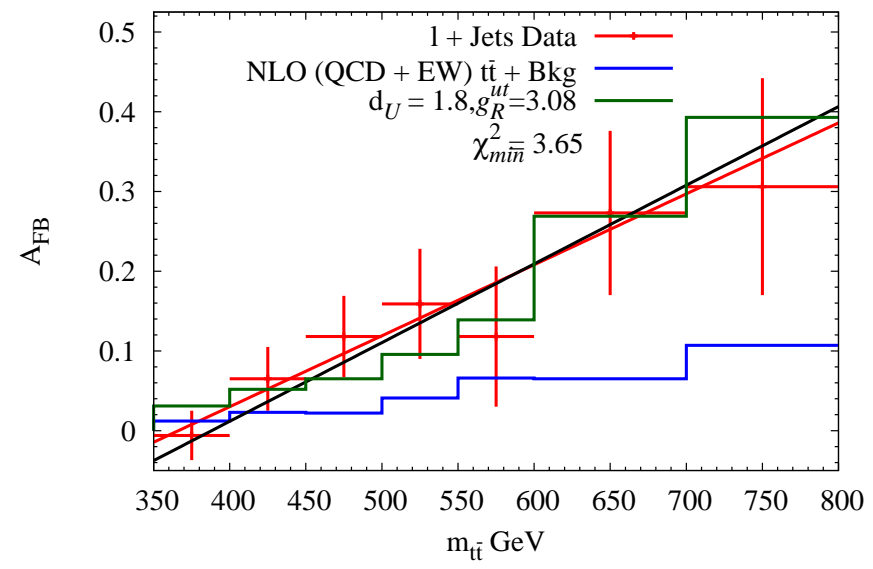

(d) FV Vector Octet: $\lambda_{R R} \neq 0$ and $\lambda_{L R}=\lambda_{R L}=\lambda_{L L}=0$

FIG. 23: $m_{t \bar{t}}$ distribution of $A_{F B}^{t \bar{t}}$ at $\chi_{\min }^{2}$. for the four favorable point-sets in parameter space at fixed $\Lambda_{\mathcal{U}}=1$ TeV. The histogram corresponding to the best-fit model parameters are shown by green steps, the experimental data points are shown with its errors in red, while the SM NLO $(Q C D+E W)$ with backgrounds are shown in the blue shaded histogram. The red straight line in all graphs is the best-fit line with the experimental data from ref. [9] while the black straight line depicts the best-fit line with the model (unparticles) plus SM NLO (QCD +EW) with backgrounds data.

of the mass gap as long as all the momentum invariants involved with the unparticle propagators are much larger than the conformal symmetry breaking scale. For $\mu=1 \mathrm{GeV}, 10 \mathrm{GeV}$ and $m_{Z}$ the effects are negligibly small. Since the minimum of these momentum invariants in case of top pair production is the threshold $m_{t \bar{t}}$, the suppression of the partonic cross-section can be at most $\left(1-\mu^{2} / \hat{s}_{\min }\right)^{d_{\mathcal{U}}-2}=\left(1-\mu^{2} / m_{t \bar{t}}\right)^{d_{\mathcal{U}}-2}$ and $\left(1-\mu^{2} / \hat{t}_{\text {min. }}\right)^{d_{\mathcal{U}}-2}=\left(1-\mu^{2} / m_{t \bar{t}}^{2}\right)^{d_{\mathcal{U}}-2}$ for $s$ and $t$ channel processes respectively. We have checked the stability and consistency of our results corresponding to the above mentioned choices of the breaking scales for all $s$ and $t$ channel processes.

Similarly computation of the same sign top pair production mediated by the flavor violating unparticles do not have any impact due to introduction of the breaking scale as long as the scale is sufficiently smaller than the same sign top pair threshold $\simeq 350 \mathrm{GeV}$.

The presence of FV couplings leads to the decay process like $t \rightarrow u+\mathcal{U}$ which diverges for $d_{\mathcal{U}}>2[18$, [50] unless one introduces a mass gap. Therefore, the adoption of scale invariance breaking is inevitable in the region $1<d_{\mathcal{U}}<2$ for the top pair production and same sign top pair production processes induced by FV couplings. However, once scale invariance is broken at a scale $\mu$, the states with momenta $p^{2}<\mu^{2}$ are removed from phase space resulting in a finite and positive decay width even for $d_{\mathcal{U}}<2[36]$.

Introduction of color octet unparticles are likely to produce them in plenty at hadron colliders through $q \bar{q} \rightarrow$ 
$\mathcal{U}^{V / T}+\mathcal{U}^{V / T}$ and $g g \rightarrow \mathcal{U}^{V / T}+\mathcal{U}^{V / T}$. These processes mediate through $s$ and $t$ channels. Gluon flux being low at TeVatron, production of such colored unparticles from gluon-gluon initial state may not have large bearing in our analysis. Production of colored scalar unparticles has been studied by Cacciapaglia et al [43]. There they showed that the pair production cross-section of color scalar unparticles is suppressed by a factor of $\left(2-d_{\mathcal{U}}\right)$ with respect to the particle pair production. One would expect the production of the vector unparticles should follow the same suit with respect to the vector particles. But then these channels are also constrained by observed di-jet cross-section at TeVatron. The upper limit on the allowed cross-section through these processes translates to an upper bound on the couplings of the respective light quark with colored vector/ tensor unparticles.

Throughout our study including the $\chi^{2}$ analysis of $m_{t \bar{t}}$ distribution of $A_{F B}^{t \bar{t}}$, the cross-sections depends on the product of couplings $g_{i}^{\mathcal{U}_{(V / T)}^{\mathrm{n}} \bar{q} q} g_{j}^{\mathcal{U}_{(V / T)}^{\mathrm{n}}{ }^{\bar{t} t}}=\lambda_{i j}^{(V / T)}$ involving light quarks and top quark with flavor conserving color vector/ tensor unparticles. In light of our assumption $g_{(L / R)}^{\mathcal{U}_{(V / T)}^{\mathrm{n}} \bar{q} q}<<g_{(L / R)}^{\mathcal{U}_{(V / T)}^{\mathrm{n}} \bar{t} t}$ ( mentioned in subsection IVA , our analysis is consistent with respect to the observation from di-jet cross-section.

Although introduction of flavor violating interactions involving colored unparticles, up quark and top quark in a $\hat{t}$ channel process do not encounter these shortcomings but it can still copiously produce unparticles in the colliders via top decay. The contribution of the decay channel to the partial top decay width constraints this coupling for $d_{\mathcal{U}}>2$.

However, all these problems can be eased out if we can model the color unparticle theory by requiring it to have an infrared cut-off. Fairly large $\mu$ is likely to suppress the copious production of color unparticles through all channels discussed here. Even this allows to constrain the couplings from the decay process $t \rightarrow u+\mathcal{U}^{V}$ in the region $1<d_{\mathcal{U}}<2$ corresponding to specific choice of $\mu$ from the measurement of the top decay width.

\section{Observations and Conclusions}

We have studied the interactions of vector and tensor color singlet/ octet unparticles in the top sector at TeVatron, through FC and FV couplings. In this way we have made an attempt to address the existing anomaly in the $A_{F B}^{t \bar{t}}$ at TeVatron keeping the cross-section and spin-correlation of $t \bar{t}$ consistent with the data. We have also studied the contribution of these unparticles to the top decay width and the same sign top pair production induced by FV couplings.

We summarize our observations here:

1. In the range of $d_{\mathcal{U}}$ allowed by completely conformal theory, i.e., $d_{\mathcal{U}}>3$, appreciable positive $A_{F B}^{t \bar{t}}$ is obtained, albeit for very high values of unparti- cle couplings which may not be in the perturbative regime.

In figure 24. we show the behaviour of cross section for the process $q \bar{q} \rightarrow t \bar{t}$ as a function of the parton center of mass energy $\sqrt{\hat{s}} \equiv m_{t \bar{t}}$ varying from the threshold value to $1 \mathrm{TeV}$. Investigating the perturbative nature of the couplings for the fully conformal theory in figure 24a, we plot the behaviour corresponding to the maximum allowed interaction strengths such that unitarity is not violated until $1 \mathrm{TeV}$. These upper limits on the interaction strengths may be read off directly from the figure e.g. the interaction strength $\sqrt{\lambda_{R R}^{V}} /\left(\Lambda_{\mathcal{U}}\right)^{d_{\mathcal{U}}-1}<200(\mathrm{TeV})^{1-d_{\mathcal{U}}}$ in case of FC vector singlet. For a given $d_{\mathcal{U}}$, this limit can translate into an upper bound on the coupling for fixed $\Lambda_{\mathcal{U}}$ or a lower bound on $\Lambda_{\mathcal{U}}$ for a fixed $\sqrt{\lambda_{R R}^{V}}$. Although these values contribute to the cross section $\sigma^{t \bar{t}}$ within the experimental limits but are unable to explain the observed large value of $A_{F B}^{t \bar{t}}$. Further, as mentioned earlier, at such high values of $d_{\mathcal{U}}$, the SM contact interactions cannot be ignored. Hence these values of $d_{\mathcal{U}}$ are not phenomenologically interesting.

2. The $A_{F B}^{t \bar{t}}$ anomaly in top pair production can be explained by the process mediated by $\mathrm{FC}$ vector color singlet unparticles when only the $\mathrm{RH}$ (or LH) couplings are present (i.e. the case(a) or (b) in our article). When $1<d_{\mathcal{U}}<2$, one gets appreciable positive $A_{F B}^{t \bar{t}}$ keeping the other observables, namely, $\sigma^{t \bar{t}}$ and $C^{t \bar{t}}$ within the CDF observed values. $A_{F B}^{t \bar{t}}$ is higher for values closer to $d_{\mathcal{U}}=1$.

3. In case of FC vector color octet, the cases (a) and (b) give favorable values of $A_{F B}^{t \bar{t}}$ for a large range of $d_{\mathcal{U}}$ values in the region $1<d_{\mathcal{U}}<2$ and for the couplings that do not modify the cross section and spin correlation appreciably from the SM values. Higher the value of $d_{\mathcal{U}}$, smaller is the value of $A_{F B}^{t \bar{t}}$ for a given coupling. If the $\mathrm{FC}$ octet unparticles have axial vector couplings $(\operatorname{case}(\mathrm{d}))$ then positive $A_{F B}^{t \bar{t}}$ is obtained only for $1.5<d_{\mathcal{U}}<2$. To explain the $A_{F B}^{t \bar{t}}$ anomaly for $d_{\mathcal{U}}$-values in the region $1<d_{\mathcal{U}}<1.5$ for case $(\mathrm{d})$, one has to assume that couplings of unparticle with light quarks are of opposite sign to those with top quark, just like in the case of axigluon models. With this non-universal choice of couplings, appreciable positive $A_{F B}^{t \bar{t}}$ may be obtained even for $3<d_{\mathcal{U}}<3.5$.

4. With unparticle operators assumed to be vector having FC couplings, whether being singlet or octet under $S U(3)_{C}$, the spin FB asymmetry $C_{F B}^{t \bar{t}}=A_{F B}^{t \bar{t}}$

5. Additional factors of $\beta_{t}$ and $\Lambda_{\mathcal{U}}^{-2}$ in the cross section suppress the tensor unparticle effects compared to 


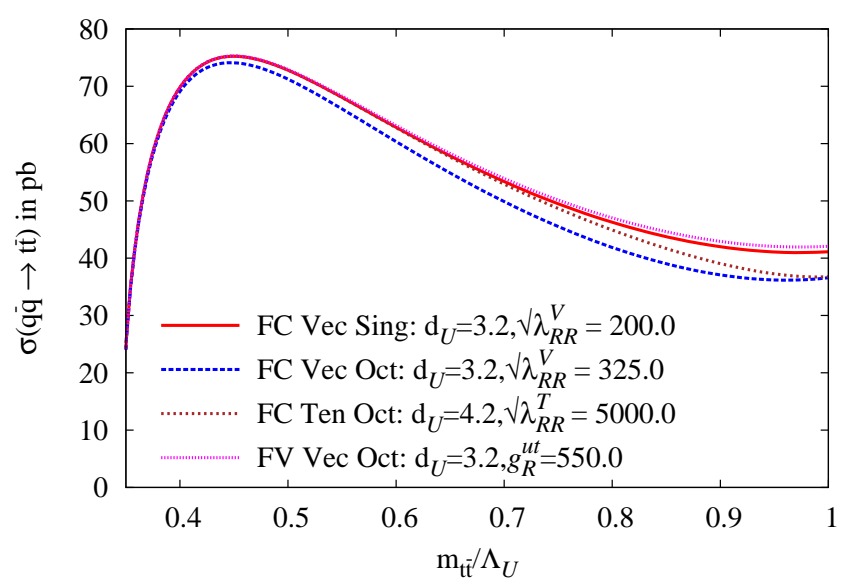

(a) $d_{\mathcal{U}}$ values allowed by completely conformal theory

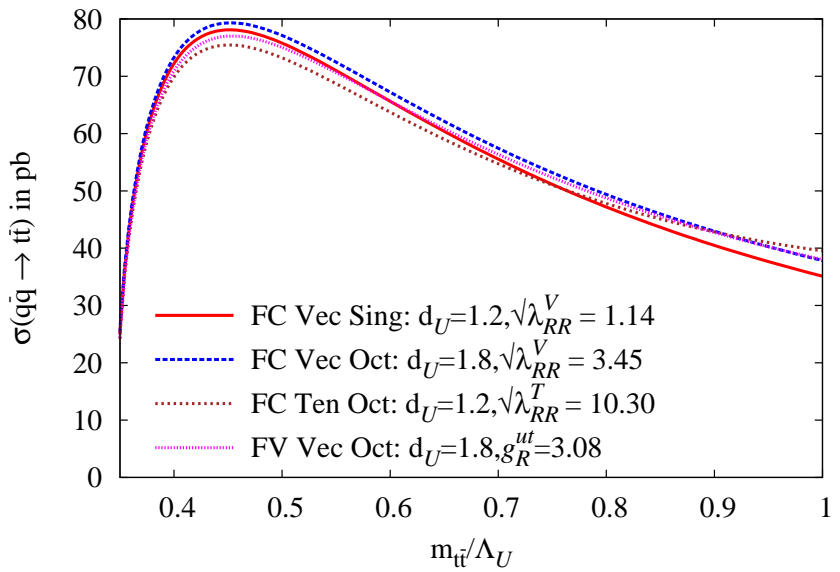

(b) Four favorable point sets based on $\chi_{\min }^{2}$

FIG. 24: Variation of the cross-section for the process $q \bar{q} \rightarrow t \bar{t}$ with $\sqrt{\hat{s}} \equiv m_{t \bar{t}}$ in units of $\Lambda_{\mathcal{U}}$. Panel (a) gives the behaviour corresponding to the maximum allowed interaction strengths such that unitarity is not violated until 1 TeV. Panel (b) shows the $\sqrt{\hat{s}}$ variation for the four focus points identified in figure 23 illustrating that the parameter values that explain the $A_{F B}^{t \bar{t}}$ anomaly preserve unitarity.

vector unparticles. Assuming the tensor unparticle to be FC singlet, there is no parameter space that can explain the $A_{F B}^{t \bar{t}}$ anomaly at the same time keeping the other observables within the experimental limits. If, however, tensor unparticles are color octets, appreciable $A_{F B}^{t \bar{t}}$ is obtained for cases (a) and (c) with $1<d_{\mathcal{U}}<1.5$ (closer is $d_{\mathcal{U}}$ to 1 , higher is $\left.A_{F B}^{t \bar{t}}\right)$. Moreover, with the presence of tensor unparticles, $C_{F B}^{t \bar{t}} \neq A_{F B}^{t \bar{t}}$.

6. In the presence of FV couplings of vector unparticle involving first and third generation quarks only, color octet unparticle gives the appreciable positive $A_{F B}^{t \bar{t}}$ for $1<d_{\mathcal{U}}<2$. Color singlet gives sufficiently positive $A_{F B}^{t \bar{t}}$ only for values of $d_{\mathcal{U}}$ very close to 1 . With both same and opposite helicity amplitudes contributing to $A_{F B}^{t \bar{t}}$ in presence of FV couplings, $A_{F B}^{t \bar{t}} \neq C_{F B}^{t \bar{t}}$.

7. We observe that although a large parameter region in FV sector is consistent with $\mathrm{CDF}$ and $\mathrm{D} \varnothing$ data, it gets constrained from the same sign top/anti-top production.

Considering the recent measurement of the top decay width, the parameter region shrinks to a large extent. Unparticles are likely to escape the detection accounting for the missing energy and transverse momentum or they will show up as pair of light quark jets/leptons. Then one would like to compare these processes with the experimentally constrained FCNC partial decay width $t \rightarrow Z+j e t$, which is $\leq 3.7 \%$ from CDF 55 and $\leq 3.2 \%$ from $\mathrm{D} \varnothing$ [56] respectively. However, the partial decay width of $t \rightarrow \mathcal{U} u$ is expected to be larger than $t \rightarrow Z+j e t$ as it's phase space allows the continuum spectrum for unparticles unlike $Z$ boson.

8. Our results and analysis are consistent with the inception of non-zero infrared cut-off as long as $\mu^{2} \ll$ momentum invariants involving unparticles in all $\hat{s}$, $\hat{t}$ and $\hat{u}$ channels at the parton level.

9. We have identified some focus points of the model which can explain the $A_{F B}^{t \bar{t}}$ anomaly (given in table III) based on the $\chi^{2}$ analysis performed with two independent parameters of the theory $d_{\mathcal{U}}$ and the coupling $\sqrt{\lambda_{i j}} / g_{i}^{u t}$ for a given $\Lambda_{\mathcal{U}}$. Following the scanning of the unconstrained parameter region we computed the $\chi_{\text {min. }}^{2}$ w.r.t the deviation from the experimentally observed $m_{t \bar{t}}$ distribution of $A_{F B}^{t \bar{t}} \quad$ spread over seven bins at the TeVatron for fixed $\Lambda_{\mathcal{U}}=1 \mathrm{TeV}$. We find that all four points corresponding to the $\chi_{\min }^{2}$. neither conflict with the other measured observables of $p \bar{p} \rightarrow t \bar{t}$ nor do they transgress the allowed upper limit of same sign top pair production cross-section and the observed total top decay width.

We investigated further and established that all these focus points preserved the unitarity which is illustrated in figure $24 \mathrm{~b}$

10. The new physics effect can either show up at the top pair production or in the top decay channels. However, in an experiment the top and anti-top are reconstructed from all observed decay products using the SM template which contains the SM $t W b$ vertices. Therefore unparticle induced decay channels of top/antitop to the visible spectrum and missing energy will add up to the cross-section reported in 
the experiment. We have, in this article, considered the unpartcle contribution only to the top pair production with SM decay of top/antitop. The total cross section including the additional top/antitop decay via unparticles is likely to reach the present upper bound of allowed one sigma band at much lower values of the couplings in comparison to that shown in figures 2, 5, 810, 13, 16a and 17.

11. The inclusion of the down sector in the Lagrangian A.4 induces the $b q \mathcal{U}$ vertices in various modes $B$ decay and in the $b \bar{b}$ production at the hadron colliders. Since our analysis involves the flavor violation among the first and third generation quarks, the flavor-violating couplings from rare decay modes $B^{0} \rightarrow K^{0} \bar{K}^{0}$ and $B^{ \pm, 0} \rightarrow \phi \pi^{ \pm, 0}$ (which have only $b \rightarrow d$ penguin contributions in the SM) constrains the left chiral $b d \mathcal{U}$ couplings. [57] The study of $C P$ phase of $B$ meson mixing also constraints the left chiral vector unparticle current [58]. SU(2) gauge invariance would then impose the constraints on the left chiral couplings in the up quarks sector as well. Thus the case with the pure right handed couplings remains unconstrained. Therefore our analysis for the flavor violating focus point which arises from the combination involving $g_{L}^{u t}=0$ and $g_{R}^{u t} \neq 0$ remains unconstrained from the $B$ physics.
It is worthwhile to probe the contribution of unparticles at LHC and correlate findings from the top sector at TeVatron. We expect that the present and forthcoming measurements with higher luminosity data for top pair cross section, charge asymmetry and spin correlation will severely constrain the parameter space of flavor conserving and violating unparticle interactions. In addition the $m_{t \bar{t}}$ distribution of the cross section [59, 60] and charge asymmetry will contrain the new physics possiblitities. With the improved $b$-tagging efficiency, the unparticle contribution to the $b \bar{b}$ production is likely to influence the model analysis. The contribution of unparticles to the light quark dijet production is likely to put an upper bound on the flavor conserving light quark-unparticle interaction strength 61 63].

Recently the measurement of same sign top pairs at CMS has ruled out the favored $Z^{\prime}$ model of $A_{F B}^{t \bar{t}}$ [52]. Therefore one expects that the same sign top pair production through unparticles will narrow down the allowed flavor violating parameter space. In the same spirit, the impact of the constraints on the FCNC decays of top quarks observed at ATLAS [64] and CMS [65] needs to be studied in this model.

Presently this analysis and an estimate of unparticle contribution to all these processes for LHC is in progress.

\begin{tabular}{|c|c|c|c|c|c|c|}
\hline$m_{t \bar{t}}$ & $A_{F B}^{t t}( \pm$ stat. $)$ & $\begin{array}{c}\mathrm{NLO}(\mathrm{QCD}+\mathrm{EW}) \\
t \bar{t}+\mathrm{Bkg}\end{array}$ & $\begin{array}{c}\text { FC Vector Singlet } \\
\text { case (a) }\end{array}$ & $\begin{array}{c}\text { FC Vector Octet } \\
\text { case (a) }\end{array}$ & $\begin{array}{c}\text { FC Tensor Octet } \\
\text { case }(d)\end{array}$ & $\begin{array}{c}\text { FV Vector Octet } \\
\text { case (a) }\end{array}$ \\
\hline$<400 \mathrm{GeV} / \mathrm{c}^{2}$ & $-0.006 \pm 0.031$ & 0.012 & 0.03 & 0.03 & 0.03 & 0.03 \\
\hline $400-450 \mathrm{GeV} / \mathrm{c}^{2}$ & $0.065 \pm 0.040$ & 0.023 & 0.05 & 0.05 & 0.06 & 0.05 \\
\hline $450-500 \mathrm{GeV} / \mathrm{c}^{2}$ & $0.118 \pm 0.051$ & 0.022 & 0.06 & 0.06 & 0.07 & 0.07 \\
\hline $500-550 \mathrm{GeV} / \mathrm{c}^{2}$ & $0.159 \pm 0.069$ & 0.041 & 0.09 & 0.10 & 0.10 & 0.10 \\
\hline $550-600 \mathrm{GeV} / \mathrm{c}^{2}$ & $0.118 \pm 0.088$ & 0.066 & 0.13 & 0.13 & 0.15 & 0.14 \\
\hline $600-700 \mathrm{GeV} / \mathrm{c}^{2}$ & $0.273 \pm 0.103$ & 0.065 & 0.21 & 0.26 & 0.29 & 0.27 \\
\hline$\geq 700 \mathrm{GeV} / \mathrm{c}^{2}$ & $0.306 \pm 0.136$ & 0.107 & 0.28 & 0.34 & 0.41 & 0.39 \\
\hline$\chi_{\min }^{2}$ & - & - & 2.80 & 3.16 & 3.36 & 3.65 \\
\hline $\begin{array}{c}\text { Slope of } \\
\text { Best-Fit Line }\end{array}$ & $(8.9 \pm 2.3) \times 10^{-4}$ & $(2.2 \pm 2.3) \times 10^{-4}$ & $6.7 \times 10^{-4}$ & $8.8 \times 10^{-4}$ & $1 \times 10^{-3}$ & $9.8 \times 10^{-4}$ \\
\hline
\end{tabular}

TABLE II: The first three columns give the bin limits of the $m_{t \bar{t}}$, the observed $A_{F B}^{t \bar{t}}$ with error and the $N L O(Q C D+E W)$ generated $A_{F B}^{t \bar{t}}$ respectively [Y]. The next four consecutive columns provide the differential $A_{F B}^{t \bar{t}}$ corresponding to the model parameters (given in figure 23) leading to $\chi_{\min }^{2}$. at fixed $\Lambda_{\mathcal{U}}=1 \mathrm{TeV}$. The penultimate line gives the $\chi_{\min }^{2}$ for respective cases. The last line in the table gives the slope of the best fit line with the simulated data.

\section{Acknowledgments}

The authors would like to thank D. Choudhury, Mukesh Kumar and A. Goyal for fruitful discussions. We acknowledge the partial support from DST, India under grant SR/S2/HEP-12/2006. SD and RI will like to ac- knowledge the UGC research award and CSIR JRF respectively for the partial financial support. MD would like to thank the IUCAA, Pune for hospitality while part of this work was completed. We also thank RECAPP, HRI for local hospitality where this work was initiated. We would like to thank D. Ghosh and M. Perez-Victoria 
for valuable comments.

\section{Appendix A: Computation of Helicity Amplitudes}

The generalized Lagrangian for SM and the $J=1,2$ neutral current via color singlets and octets are given as

$$
\mathcal{L}_{\mathrm{QCD}}=-g_{s}\left\{T^{a}\right\}_{j i} A_{\mu}^{a} \sum_{f} \bar{q}_{f j} \gamma^{\mu} q_{f i},
$$

where $g_{s}$ is the QCD coupling, $f$ is the flavor index and $i, j$ are the color indices.

$$
\begin{aligned}
\mathcal{L}_{\mathrm{NC}}=- & \sum_{f} \bar{q}_{f} \gamma^{\mu}\left[e Q_{f} A_{\mu}\right. \\
& \left.+\frac{e}{s_{W} c_{W}}\left(T_{f}^{3} P_{L}-s_{W}^{2} Q_{f}\right) Z_{\mu}\right] q_{f},
\end{aligned}
$$

where $e=$ electromagnetic coupling, $Q_{f}=$ charge of quark $q_{f}$ in units of $e, s_{W}=\sin \theta_{W}, c_{W}=\cos \theta_{W}, \theta_{W}=$ Weinberg angle.

$$
\begin{gathered}
\mathcal{L}_{J=1}^{(s)}=\frac{g_{s}}{\Lambda_{\mathcal{U}}^{d_{\mathcal{U}}-1}} \sum_{\substack{\alpha=L, R \\
\mathbf{n = 1 , 8}}} g_{\alpha}^{\mathcal{U}_{V}^{\mathbf{n}} \bar{q} q}\left\{T_{\mathbf{n}}^{a}\right\}_{j i} \mathcal{O}_{\mu}^{\mathbf{n}, a} \\
\quad \times \sum_{f} \bar{q}_{f j} \gamma^{\mu} P_{\alpha} q_{f i}+\text { h.c. } \\
\mathcal{L}_{J=1}^{(t)}=\frac{g_{s}}{\Lambda_{\mathcal{U}}^{d_{\mathcal{U}}-1}} \sum_{\substack{\alpha=L, R \\
\mathbf{n}=\mathbf{1}, \mathbf{8}}} \mathcal{O}_{\mu}^{\mathbf{n}, a}\left\{T_{\mathbf{n}}^{a}\right\}_{j i}\left[g_{\alpha}^{\mathcal{U}_{V}^{\mathbf{n}} \bar{t} u} \bar{t}_{j} \gamma^{\mu} P_{\alpha} q_{f i}\right. \\
\left.+g_{\alpha}^{\mathcal{U}_{V}^{\mathbf{n}} \bar{b} u} \bar{b}_{j} \gamma^{\mu} P_{\alpha} q_{f i}+h . c .\right]
\end{gathered}
$$

$$
\begin{aligned}
\mathcal{L}_{J=2}^{(s)} & =\frac{-g_{s}}{4 \Lambda_{\mathcal{U}}^{d \mathcal{U}}} \sum_{\substack{\alpha=L, R \\
\mathbf{n}=\mathbf{1}, \mathbf{8}}} g_{\alpha}^{\mathcal{U}_{T}^{\mathbf{n}} \bar{q} q}\left\{T_{\mathbf{n}}^{a}\right\}_{j i} \mathcal{O}_{\mu \nu}^{\mathbf{n}, a} \\
& \times \sum_{f} \bar{q}_{f j} i\left(\gamma_{\mu} \partial_{\nu}+\gamma_{\nu} \partial_{\mu}\right) P_{\alpha} q_{f i}+\text { h.c. }
\end{aligned}
$$

$$
\begin{aligned}
\mathcal{L}_{J=2}^{(t)}= & \frac{-g_{s}}{4 \Lambda_{\mathcal{U}}^{d_{\mathcal{U}}}} \sum_{\substack{\alpha=L, R \\
\mathbf{n}=\mathbf{1}, \mathbf{8}}} \mathcal{O}_{\mu \nu}^{\mathbf{n}, a}\left\{T_{\mathbf{n}}^{a}\right\}_{j i} \\
& \times i\left[g_{\alpha}^{\mathcal{U}_{T}^{\mathbf{n}} \bar{t} u} \bar{t}_{j}\left(\gamma_{\mu} \partial_{\nu}+\gamma_{\nu} \partial_{\mu}\right) P_{\alpha} q_{f i}\right. \\
& \left.+g_{\alpha}^{\mathcal{U}_{T}^{\mathbf{n}} \bar{b} u} \bar{b}_{j}\left(\gamma_{\mu} \partial_{\nu}+\gamma_{\nu} \partial_{\mu}\right) P_{\alpha} q_{f i}+\text { h.c }\right]
\end{aligned}
$$

The matrix element is computed in this appendix fixing the color flow of the particles involved in the process $q \bar{q} \rightarrow$ $t \bar{t}$ as following

$$
\left\{q\left(p_{q}\right)\right\}_{i}\left\{\bar{q}\left(p_{\bar{q}}\right)\right\}_{j} \rightarrow\left\{t\left(p_{t}\right)\right\}_{k}\left\{\bar{t}\left(p_{\bar{t}}\right)\right\}_{l}
$$

The matrix elements corresponding to the given Lagrangian in eq. A.1 - A.6

$$
\begin{aligned}
\sum_{i} i \mathcal{M}_{i}= & i \mathcal{M}_{1}(\mathrm{QCD})+i \mathcal{M}_{2}(\mathrm{NC})+i \mathcal{M}_{3}(\hat{s} ; J=1 ; \mathbf{1})+i \mathcal{M}_{4}(\hat{s} ; J=1 ; \mathbf{8})+i \mathcal{M}_{5}(\hat{t} ; J=1 ; \mathbf{1})+i \mathcal{M}_{6}(\hat{t} ; J=1 ; \mathbf{8}) \\
& +i \mathcal{M}_{7}(\hat{s} ; J=2 ; \mathbf{1})+i \mathcal{M}_{8}(\hat{s} ; J=2 ; \boldsymbol{8})+i \mathcal{M}_{9}(\hat{t} ; J=2 ; \mathbf{1})+i \mathcal{M}_{10}(\hat{t} ; J=2 ; \mathbf{8})
\end{aligned}
$$

$$
\begin{gathered}
\sum_{i} i \mathcal{M}_{i}=C_{\mathbf{Q C D}}\left[\bar{v}\left(p_{\bar{q}}, \lambda_{\bar{q}}\right)_{i} i \gamma^{\mu} u\left(p_{q}, \lambda_{q}\right)_{l}\right] \frac{-i g_{\mu \nu}}{\hat{s}}\left[\bar{u}\left(p_{t}, \lambda_{t}\right)_{k} i \gamma^{\nu} v\left(p_{\bar{t}}, \lambda_{\bar{t}}\right)_{j}\right] \\
+C_{\mathbf{N C}}\left[\bar{v}\left(p_{\bar{q}}, \lambda_{\bar{q}}\right)_{i} i \gamma^{\mu} u\left(p_{q}, \lambda_{q}\right)_{l}\right] \frac{-i g_{\mu \nu}}{\hat{s}}\left[\bar{u}\left(p_{t}, \lambda_{t}\right)_{k} i \gamma^{\nu} v\left(p_{\bar{t}}, \lambda_{\bar{t}}\right)_{j}\right] \\
+C_{\mathbf{N C}}\left[\bar{v}\left(p_{\bar{q}}, \lambda_{\bar{q}}\right)_{i} i \gamma^{\mu}\left(T_{q}^{3} P_{L}-s_{W}^{2} Q_{q}\right) u\left(p_{q}, \lambda_{q}\right)_{l}\right] \frac{-i g_{\mu \nu}}{\hat{s}-m_{Z}^{2}+i m_{Z} \Gamma_{Z}}\left[\bar{u}\left(p_{t}, \lambda_{t}\right)_{k} i \gamma^{\nu}\left(T_{t}^{3} P_{L}-s_{W}^{2} Q_{t}\right) v\left(p_{\bar{t}}, \lambda_{\bar{t}}\right)_{j}\right] \\
+\sum_{\mathbf{n} \equiv \mathbf{1}, \mathbf{8}} C_{\mathbf{n}}^{s}\left[\bar{v}\left(p_{\bar{q}}, \lambda_{\bar{q}}\right)_{i} \frac{i \gamma^{\mu}}{\Lambda^{d_{\mathcal{U}}-1}}\left(g_{L}^{\mathcal{U}_{V}^{\mathbf{n}} \bar{q} q} P_{L}+g_{R}^{\mathcal{U}_{V}^{\mathbf{n}} \bar{q} q} P_{R}\right) u\left(p_{q}, \lambda_{q}\right)_{l}\right] \frac{i A_{d_{\mathcal{U}}}(-\hat{s})^{d_{\mathcal{U}}-2}}{2 \sin \left(d_{\mathcal{U}} \pi\right)}\left[g_{\mu \nu}-a \frac{\left(p_{q}+p_{\bar{q}}\right)^{\mu}\left(p_{q}+p_{\bar{q}}\right)^{\nu}}{\left(p_{q}+p_{\bar{q}}\right)^{2}}\right] \\
\times\left[\bar{u}\left(p_{t}, \lambda_{t}\right)_{k} \frac{i \gamma^{\nu}}{\Lambda^{d_{\mathcal{U}}-1}}\left(g_{L}^{\mathcal{U}_{V}^{\mathbf{n}} \bar{t} t} P_{L}+g_{R}^{\mathcal{U}_{V}^{\mathbf{n}} \bar{t} t} P_{R}\right) v\left(p_{\bar{t}}, \lambda_{\bar{t}}\right)_{j}\right]
\end{gathered}
$$




$$
\begin{aligned}
& -\sum_{\mathbf{n} \equiv \mathbf{1}, \mathbf{8}} C_{\mathbf{n}}^{t}\left[\bar{v}\left(p_{\bar{q}}, \lambda_{\bar{q}}\right)_{i} \frac{i \gamma^{\mu}}{\Lambda^{d_{\mathcal{U}}-1}}\left(g_{L}^{\mathcal{U}_{V}^{\mathrm{n}} \bar{t} \bar{q}} P_{L}+g_{R}^{\mathcal{U}_{V}^{\mathbf{n}} \bar{t} \bar{q}} P_{R}\right) v\left(p_{\bar{t}}, \lambda_{\bar{t}}\right)_{j}\right] \frac{i A_{d_{\mathcal{U}}}(-\hat{t})^{d_{\mathcal{U}}-2}}{2 \sin \left(d_{\mathcal{U}} \pi\right)}\left[g_{\mu \nu}-a \frac{\left(p_{q}-p_{t}\right)^{\mu}\left(p_{q}-p_{t}\right)^{\nu}}{\left(p_{q}-p_{t}\right)^{2}}\right] \\
& \times\left[\bar{u}\left(p_{t}, \lambda_{t}\right)_{k} \frac{i \gamma^{\nu}}{\Lambda^{d \mathcal{u}-1}}\left(g_{L}^{\mathcal{U}_{V}^{\mathrm{n}} t q} P_{L}+g_{R}^{\mathcal{U}_{V}^{\mathrm{n}} t q} P_{R}\right) q\left(p_{q}, \lambda_{q}\right)_{l}\right] \\
& +\sum_{\mathbf{n} \equiv \mathbf{1}, \mathbf{8}} C_{\mathbf{n}}^{s}\left[\bar{v}\left(p_{\bar{q}}, \lambda_{\bar{q}}\right)_{i} \frac{-i\left[\gamma^{\mu}\left(p_{q}-p_{\bar{q}}\right)^{\nu}+\gamma^{\nu}\left(p_{q}-p_{\bar{q}}\right)^{\mu}\right]}{4 \Lambda^{d_{\mathcal{U}}}}\left(g_{L}^{\mathcal{U}_{T}^{\mathrm{n}} \bar{q} q} P_{L}+g_{R}^{\mathcal{U}_{T}^{\mathrm{n}} \bar{q} q} P_{R}\right) u\left(p_{q}, \lambda_{q}\right)_{l}\right] \frac{i A_{d_{\mathcal{U}}}(-\hat{s})^{d_{\mathcal{U}}-2}}{2 \sin \left(d_{\mathcal{U}} \pi\right)} \mathcal{T}_{\mu \nu, \alpha \beta} \\
& \times\left[\bar{u}\left(p_{t}, \lambda_{t}\right)_{k} \frac{i\left[\gamma^{\alpha}\left(p_{t}-p_{\bar{t}}\right)^{\beta}+\gamma^{\beta}\left(p_{t}-p_{\bar{t}}\right)^{\alpha}\right]}{4 \Lambda^{d \mathcal{U}}}\left(g_{L}^{\mathcal{U}_{T}^{\mathrm{n}} \bar{t} t} P_{L}+g_{R}^{\mathcal{U}_{T}^{\mathrm{n}} \bar{t} t} P_{R}\right) v\left(p_{\bar{t}}, \lambda_{\bar{t}}\right)_{j}\right] \\
& -\sum_{\mathbf{n} \equiv \mathbf{1}, \mathbf{8}} C_{\mathbf{n}}^{t}\left[\bar{v}\left(p_{\bar{q}}, \lambda_{\bar{q}}\right)_{i} \frac{-i\left[\gamma^{\mu}\left(p_{t}+p_{q}\right)^{\nu}+\gamma^{\nu}\left(p_{t}+p_{q}\right)^{\mu}\right]}{4 \Lambda^{d_{\mathcal{U}}}}\left(g_{L}^{\mathcal{U}_{T}^{\mathrm{n}} \bar{t} \bar{q}} P_{L}+g_{R}^{\mathcal{U}_{T}^{\mathrm{n}} \overline{\bar{q}} \overline{\bar{q}}} P_{R}\right) v\left(p_{\bar{t}}, \lambda_{\bar{t}}\right)_{j}\right] \frac{i A_{d_{\mathcal{U}}}(-\hat{t})^{d_{\mathcal{U}}-2}}{2 \sin \left(d_{\mathcal{U}} \pi\right)} \mathcal{T}_{\mu \nu, \alpha \beta} \\
& \times\left[\bar{u}\left(p_{t}, \lambda_{t}\right)_{k} \frac{i\left[\gamma^{\alpha}\left(p_{\bar{q}}+p_{\bar{t}}\right)^{\beta}+\gamma^{\beta}\left(p_{\bar{q}}+p_{\bar{t}}\right)^{\alpha}\right]}{4 \Lambda^{d_{\mathcal{U}}}}\left(g_{L}^{\mathcal{U}_{T}^{\mathrm{n}} t q} P_{L}+g_{R}^{\mathcal{U}_{T}^{\mathrm{n}} t q} P_{R}\right) q\left(p_{q}, \lambda_{q}\right)_{l}\right]
\end{aligned}
$$

Here $C_{\mathbf{n}}^{\mathbf{s}}$ is the color propagator for the color octet vectors/tensors in $s$ channel and $C_{\mathbf{n}}^{t}$ is color propagator for the color octet $(n \equiv 8)$ and singlet $(n \equiv 1)$ vectors/tensors in $t$ channel. To compute the squared and interference terms with these color propagators, we provide the color factors in the following table.

We compute the helicity amplitudes in the center of mass frame $q \bar{q}$. The momentum assignments are

$$
\begin{gathered}
p_{q}=\frac{\sqrt{\hat{s}}}{2}(1,0,0,1), \quad p_{\bar{q}}=\frac{\sqrt{\hat{s}}}{2}(1,0,0,-1), \\
p_{t}=\frac{\sqrt{\hat{s}}}{2}\left(1, \beta_{t} s_{\theta}, 0, \beta_{t} c_{\theta}\right), \quad p_{\bar{t}}=\frac{\sqrt{\hat{s}}}{2}\left(1,-\beta_{t} s_{\theta}, 0,-\beta_{t} c_{\theta}\right)
\end{gathered}
$$

where $s_{\theta} \equiv \sin \theta ; c_{\theta} \equiv \cos \theta, \beta_{t}=\sqrt{1-\frac{4 m_{t}^{2}}{\hat{s}}}$ and $\theta$ is the angle between $q$ and $t$ momenta.

\section{Helicity amplitudes for $q \bar{q} \rightarrow t \bar{t}$ in Standard Model}

The helicity amplitudes for $q \bar{q} \rightarrow t \bar{t}$ in Standard Model are given by

$$
\begin{gathered}
\mathcal{M}_{1,2}(+- \pm \pm)= \\
\mp g_{R}\left[g_{L}+g_{R}\right] \frac{1}{2} \sqrt{1-\beta_{t}^{2}} s_{\theta} \\
\mathcal{M}_{1,2}(+- \pm \mp)= \\
\pm g_{R}\left[g_{L}\left(1 \mp \beta_{t}\right)+g_{R}\left(1 \pm \beta_{t}\right)\right] \frac{1}{2}\left(1 \pm c_{\theta}\right)
\end{gathered}
$$

$$
\begin{gathered}
\mathcal{M}_{1,2}(-+ \pm \pm)= \\
\mp g_{L}\left[g_{L}+g_{R}\right] \frac{1}{2} \sqrt{1-\beta_{t}^{2}} s_{\theta} \\
\mathcal{M}_{1,2}(-+ \pm \mp)= \\
\mp g_{L}\left[g_{L}\left(1 \mp \beta_{t}\right)+g_{R}\left(1 \pm \beta_{t}\right)\right] \frac{1}{2}\left(1 \mp c_{\theta}\right) .
\end{gathered}
$$

In above if the helicity amplitudes are gluon mediating then they are in units of $g_{s}^{2}$, if photon mediating then they are in units of $\left(e Q_{q}\right)\left(e Q_{t}\right)$ and if $Z$-boson mediating then they are in units of $\left(e / s_{W} c_{W}\right)^{2} s /\left(s-m_{Z}^{2}\right)$. Here for gluon and photon mediating process $g_{L}=$ $g_{R}=1$ whereas for $Z$-boson mediating process $g_{L}=$ $T_{q}^{3}-s_{W}^{2} Q_{q}, g_{R}=-s_{W}^{2} Q_{q}$.

\section{Helicity amplitudes for $q \bar{q} \rightarrow t \bar{t}$ via flavor conserving vector unparticles}

The helicity amplitudes mediated by the flavor conserving vector unparticle $\mathcal{M}_{3,4}(\hat{s} ; J=1 ; \mathbf{n})$ in units of $g_{s}^{2}(-1)^{d_{\mathcal{U}}-3} \frac{A_{d_{\mathcal{U}}}}{2 \sin \left(d_{\mathcal{U}} \pi\right)}\left[\frac{\hat{s}}{\Lambda_{\mathcal{U}}^{2}}\right]^{d_{\mathcal{U}}-1}$ are given by

$$
\begin{gathered}
\mathcal{M}_{3,4}(+- \pm \pm)= \\
\mp g_{R}^{\mathcal{U}_{V}^{\mathrm{n}} \bar{q} q}\left[g_{L}^{\mathcal{U}_{V}^{\mathrm{n}} \bar{t} t}+g_{R}^{\mathcal{U}_{V}^{\mathrm{n}} \bar{t} t}\right] \frac{1}{2} \sqrt{1-\beta_{t}^{2}} s_{\theta} \quad(\mathrm{A} .15) \\
\mathcal{M}_{3,4}(+- \pm \mp)= \\
\pm g_{R}^{\mathcal{U}_{V}^{\mathrm{n}} \bar{q} q}\left[g_{L}^{\mathcal{U}_{V}^{\mathrm{n}} \bar{t} t}\left(1 \mp \beta_{t}\right)+g_{R}^{\mathcal{U}_{V}^{\mathrm{n}} \bar{t} t}\left(1 \pm \beta_{t}\right)\right] \frac{1}{2}\left(1 \pm c_{\theta}\right)
\end{gathered}
$$$$
\mathcal{M}_{3,4}(+- \pm \mp)=
$$ 


\begin{tabular}{|c|c|c|c|c|}
\hline \hline Color Factor & FC Octet & FV Octet & FC singlet & FV singlet \\
$q\left(p_{q}\right)_{i} \bar{q}\left(p_{\bar{q}}\right)_{j} \rightarrow$ & $\{j, i\} \quad\{k, l\}$ & $\{i, k\} \quad\{l, j\}$ & $\{j, i\} \quad\{k, l\}$ & $\{i, k\} \quad\{l, j\}$ \\
$t\left(p_{t}\right)_{k} \bar{t}\left(p_{\bar{t}}\right)_{l}$ & $s$ chan. & $t$ chan. & $s$ chan. & $t$ chan. \\
& $C_{\mathbf{8}}^{s}=C_{\mathbf{Q C D}}$ & $C_{\mathbf{8}}^{t}$ & $C_{\mathbf{1}}^{s}=C_{\mathbf{N C}}$ & $C_{\mathbf{1}}^{t}$ \\
\hline Int. with QCD & 2 & $-2 / 3$ & 0 & 4 \\
\hline Int. with NC & 0 & 0 & 9 & 3 \\
\hline Squared term & 2 & 2 & 9 & 9 \\
\hline \hline
\end{tabular}

TABLE III: Color Factors for the interference and the squared terms of $s$ and $t$ channels for color singlet and octet vectors and tensors.

$$
\begin{aligned}
& \mathcal{M}_{3,4}(-+ \pm \pm)=\left.\mathcal{M}_{3,4}(+- \pm \pm)\right|_{L \leftrightarrow R} \\
& \mathcal{M}_{3,4}(-+ \pm \mp)=\left.\mathcal{M}_{3,4}(+-\mp \pm)\right|_{L \leftrightarrow R} .
\end{aligned}
$$

\section{Helicity amplitudes for $q \bar{q} \rightarrow t \bar{t}$ via flavor violating vector unparticles}

The following helicity amplitudes $\mathcal{M}_{5,6}(\hat{t} ; J=1 ; \mathbf{n})$ are given in units of $g_{s}^{2} \frac{A_{d_{\mathcal{U}}}}{2 \sin \left(d_{\mathcal{U}} \pi\right)}\left[\frac{\hat{t}}{\Lambda_{\mathcal{U}}^{2}}\right]^{d_{\mathcal{U}}-1} \frac{\hat{s}}{\hat{t}}$.

$$
\begin{aligned}
& \mathcal{M}_{5,6}(++ \pm \pm)= \\
& \pm g_{L}^{\mathcal{U}_{V}^{\mathrm{n}} \bar{t} q} g_{R}^{\mathcal{U}_{V}^{\mathrm{n}} \bar{t} q^{\prime}}\left(1 \pm \beta_{t}\right)\left[1 \pm a \frac{m_{t}^{2}}{4 \hat{t}}\left(1 \pm c_{\theta}\right)\right](\mathrm{A} \\
& \mathcal{M}_{5,6}(++ \pm \mp)= \\
& g_{L}^{\mathcal{U}_{V}^{\mathbf{n}} \bar{t} q} g_{R}^{\mathcal{U}_{V}^{\mathbf{n}} \bar{t} q^{\prime}} a \frac{m_{t}^{2}}{4 \hat{t}} \sqrt{1-\beta_{t}^{2}} s_{\theta} \\
& \mathcal{M}_{5,6}(+- \pm \pm)= \\
& -g_{R}^{\mathcal{U}_{V}^{\mathrm{n}} \bar{t} q} g_{R}^{\mathcal{U}_{V}^{\mathbf{n}} \bar{t} q^{\prime}} \sqrt{1-\beta_{t}^{2}} \frac{1}{2} s_{\theta}\left[1+a \frac{m_{t}^{2}}{4 \hat{t}}\right] \\
& \mathcal{M}_{5,6}(+- \pm \mp)= \\
& -g_{R}^{\mathcal{U}_{V}^{\mathrm{n}} \bar{t} q} g_{R}^{\mathcal{U}_{V}^{\mathrm{n}} \bar{t} q^{\prime}}\left(1 \pm \beta_{t}\right) \frac{1}{2}\left(1 \pm c_{\theta}\right)\left[1+a \frac{m_{t}^{2}}{4 \hat{t}}\right] \\
& \mathcal{M}_{5,6}(-+ \pm \pm)=\left.\mathcal{M}_{5,6}(+-\mp \mp)\right|_{L \leftrightarrow R} \\
& \mathcal{M}_{5,6}(-+ \pm \mp)=\left.\mathcal{M}_{5,6}(+-\mp \pm)\right|_{L \leftrightarrow R} \\
& \mathcal{M}_{5,6}(-- \pm \pm)=\left.\mathcal{M}_{5,6}(++\mp \mp)\right|_{L \leftrightarrow R} \\
& \mathcal{M}_{5,6}(-- \pm \mp)=\left.\mathcal{M}_{5,6}(++\mp \pm)\right|_{L \leftrightarrow R} \text {. }
\end{aligned}
$$

\section{Helicity amplitudes for $q \bar{q} \rightarrow t \bar{t}$ via flavor conserving tensor unparticles}

The following helicity amplitudes $\mathcal{M}_{7,8}(\hat{s} ; J=2 ; \mathbf{n})$ are given in units of $g_{s}^{2}(-1)^{d_{\mathcal{U}}-2} \frac{A_{d_{\mathcal{U}}}}{2 \sin \left(d_{\mathcal{U}} \pi\right)}\left[\frac{\hat{s}}{4 \Lambda_{\mathcal{U}}^{2}}\right]^{d_{\mathcal{U}}} 4 d_{\mathcal{U}}\left(d_{\mathcal{U}}-\right.$ 1).

$$
\begin{gathered}
\mathcal{M}_{7,8}(+- \pm \pm)= \\
\mp g_{R}^{\mathcal{U}_{T}^{\mathrm{n}} \bar{q} q}\left[g_{L}^{\mathcal{U}_{T}^{\mathrm{n}} \bar{t} t}+g_{R}^{\mathcal{U}_{T}^{\mathrm{n}} \bar{t} t}\right] \beta_{t} \sqrt{1-\beta_{t}^{2}} s_{\theta} c_{\theta} \quad(\mathrm{A} .27) \\
\mathcal{M}_{7,8}(+- \pm \mp)=g_{R}^{\mathcal{U}_{T}^{\mathrm{n}} \bar{q} q}\left[g_{L}^{\mathcal{U}_{T}^{\mathrm{n}} \bar{t} t}\left(1 \mp \beta_{t}\right)+g_{R}^{\mathcal{U}_{T}^{\mathrm{n}} \bar{t} t}\left(1 \pm \beta_{t}\right)\right] \\
\times \frac{1}{2} \beta_{t}\left(1 \pm c_{\theta}\right)\left(1 \mp 2 c_{\theta}\right) \\
\mathcal{M}_{7,8}(-+ \pm \pm)=\left.\mathcal{M}_{7,8}(+- \pm \pm)\right|_{L \leftrightarrow R} \quad(\mathrm{~A} .29) \\
\mathcal{M}_{7,8}(-+ \pm \mp)=\left.\mathcal{M}_{7,8}(+-\mp \pm)\right|_{L \leftrightarrow R} .(\mathrm{A} .30)
\end{gathered}
$$

\section{Helicity amplitudes for $q q \rightarrow t t$ via flavor violating vector unparticles}

The following helicity amplitudes of $t$-channel diagram are given in units of $g_{s}^{2} \frac{A_{d_{\mathcal{U}}}}{2 \sin \left(d_{\mathcal{U}} \pi\right)}\left[\frac{\hat{t}}{\Lambda_{\mathcal{U}}^{2}}\right]^{d_{\mathcal{U}}-1} \frac{\hat{s}}{\hat{t}}$. The symbol $a$ is defined in (19)

$$
\mathcal{M}_{++ \pm \pm}^{t}=-g_{R}^{q t} g_{R}^{q t}\left(1 \pm \beta_{t}\right)\left[1+a \frac{m_{t}^{2}}{p^{2}} \frac{1}{2}\left(1 \pm c_{\theta}\right)\right]
$$

$$
\begin{gathered}
\mathcal{M}_{++ \pm \mp}^{t}=g_{R}^{q t} g_{R}^{q t} \sqrt{1-\beta_{t}^{2}} \frac{1}{2} s_{\theta} a \frac{m_{t}^{2}}{p^{2}} \\
\mathcal{M}_{-+ \pm \pm}^{t}=\mp g_{L}^{q t} g_{R}^{q t} \sqrt{1-\beta_{t}^{2}} \frac{1}{2} s_{\theta}\left[1+a \frac{m_{t}^{2}}{p^{2}}\right](\mathrm{A} \\
\mathcal{M}_{-+ \pm \mp}^{t}= \pm g_{L}^{q t} g_{R}^{q t}\left(1 \mp \beta_{t}\right) \frac{1}{2}\left(1 \mp c_{\theta}\right)\left[1+a \frac{m_{t}^{2}}{p^{2}}\right]
\end{gathered}
$$




$$
\begin{aligned}
& \mathcal{M}_{+- \pm \pm}^{t}=\left.\mathcal{M}_{-+ \pm \pm}^{t}\right|_{L \rightarrow R} \\
& \mathcal{M}_{+- \pm \mp}^{t}=\left.\mathcal{M}_{-+\mp \pm}^{t}\right|_{L \rightarrow R} \\
& \mathcal{M}_{-- \pm \pm}^{t}=\left.\mathcal{M}_{++\mp \mp}^{t}\right|_{L \rightarrow R} \\
& \mathcal{M}_{-- \pm \mp}^{t}=-\left.\mathcal{M}_{++\mp \pm}^{t}\right|_{L \rightarrow R}
\end{aligned}
$$

The helicity amplitudes of $u$-channel diagram are given in units of $g_{s}^{2} \frac{A_{d_{\mathcal{U}}}}{2 \sin \left(d_{\mathcal{U}} \pi\right)}\left[\frac{\hat{t}}{\Lambda_{\mathcal{U}}^{2}}\right]^{d_{\mathcal{U}}-1} \frac{\hat{s}}{\hat{u}}$.

$$
\begin{gathered}
\mathcal{M}_{++ \pm \pm}^{u}=-\mathcal{M}^{t}+ \pm \pm\left.\right|_{\theta \rightarrow \pi+\theta} \\
\mathcal{M}_{++ \pm \mp}^{u}=-\mathcal{M}^{t}++ \pm\left.\mp\right|_{\theta \rightarrow \pi+\theta} \\
\mathcal{M}_{-+ \pm \pm}^{u}=-\mathcal{M}^{t}-+ \pm \pm\left.\right|_{\theta \rightarrow \pi+\theta} \\
\mathcal{M}_{-+ \pm \mp}^{u}=\mathcal{M}^{t}+ \pm\left.\mp\right|_{\theta \rightarrow \pi+\theta} \\
\mathcal{M}_{+- \pm \pm}^{u}=\left.\mathcal{M}_{-+ \pm \pm}^{u}\right|_{L \rightarrow R}
\end{gathered}
$$

$$
\begin{aligned}
& \mathcal{M}_{+- \pm \mp}^{u}=\left.\mathcal{M}_{-+\mp \pm}^{u}\right|_{L \rightarrow R} \\
& \mathcal{M}_{-- \pm \pm}^{u}=\left.\mathcal{M}_{++\mp \mp}^{u}\right|_{L \rightarrow R} \\
& \mathcal{M}_{-- \pm \mp}^{u}=-\left.\mathcal{M}_{++\mp \pm}^{u}\right|_{L \rightarrow R}
\end{aligned}
$$

\section{Appendix B: Partial Decay Width for $t \rightarrow q \mathcal{U}$ via flavor violating vector unparticles}

The matrix element of $t \rightarrow q \mathcal{U}$ is given by $\mathcal{M}=$ $g_{s} \Lambda_{\mathcal{U}}^{1-d_{\mathcal{U}}} \bar{u}\left(p_{q}\right) \gamma^{\mu}\left(g_{L}^{\mathcal{U}_{V}^{\mathrm{n}} t q} P_{L}+g_{R}^{\mathcal{U}_{V}^{\mathbf{n}} t q} P_{R}\right) u\left(p_{t}\right) \epsilon_{\mu}\left(p_{\mathcal{U}}\right)$

Averaging over spin and color the matrix element squared reads as

$$
\begin{aligned}
\overline{|\mathcal{M}|^{2}}= & \frac{N_{C}}{6} \frac{2 g_{s}^{2}}{\Lambda_{\mathcal{U}}^{2\left(d_{\mathcal{U}}-1\right)}}\left[\left(g_{L}^{\mathcal{U}_{V}^{\mathrm{n}} t q}\right)^{2}+\left(g_{R}^{\mathcal{U}_{V}^{\mathrm{n}} t q}\right)^{2}\right] m_{t} p_{q}^{0} \\
& \times\left[\frac{(a+2) m_{t}-4 p_{q}^{0}}{\left(m_{t}-2 p_{q}^{0}\right)}\right]
\end{aligned}
$$

The differential decay width for the top decaying to an unparticle and quark is given as

$$
\begin{aligned}
d \Gamma & =\frac{N_{C}}{6} \frac{\overline{|\mathcal{M}|^{2}}}{2 m_{t}} \frac{A_{d \mathcal{U}}}{16 \pi^{3}} \frac{p_{q}^{0} d p_{q}^{0} d \Omega}{\left(m_{t}^{2}-2 m_{t} p_{q}^{0}\right)^{2-d_{\mathcal{U}}}} \\
& =\frac{N_{C}}{6} \frac{g_{s}^{2}}{\Lambda_{\mathcal{U}}^{2\left(d_{\mathcal{U}}-1\right)}} \frac{A_{d_{\mathcal{U}}}}{16 \pi^{3}}\left[\left(g_{L}^{\mathcal{U}_{V}^{\mathrm{n}} t q}\right)^{2}+\left(g_{R}^{\mathcal{U}_{V}^{\mathrm{n}} t q}\right)^{2}\right]\left[\frac{(a+2) m_{t}^{2}-4 m_{t} p_{q}^{0}}{\left(m_{t}^{2}-2 m_{t} p_{q}^{0}\right)}\right]\left(p_{q}^{0}\right)^{2} \theta\left(m_{t}-2 p_{q}^{0}\right) d p_{q}^{0} d \Omega \\
& =\frac{N_{C}}{6} \frac{A_{d_{\mathcal{U}}}}{4 \pi^{2}} g_{s}^{2}\left[\left(g_{L}^{\mathcal{U}_{V}^{\mathrm{n}} t q}\right)^{2}+\left(g_{R}^{\mathcal{U}_{V}^{\mathrm{n}} t q}\right)^{2}\right]\left(\frac{m_{t}^{2}}{\Lambda_{\mathcal{U}}^{2}}\right)^{d_{\mathcal{U}}-1} m_{t}[(a+2)-4 x](1-2 x)^{d_{\mathcal{U}}-3} x^{2} \theta(1-2 x) d x .
\end{aligned}
$$

In (B.2) we have taken $x=p_{q}^{0} / m_{t}$. Integrating (B.2) w.r.t. $x$ in the limit $[0,1 / 2]$ we get the total decay width of top quark for $d_{\mathcal{U}}>2$. The color factor $N_{C}=3,4$ for singlet and octet unparticle respectively. On introduction of the mass gap $\mu \neq 0$ equation (B.2) becomes

$d \Gamma=\frac{N_{C}}{6} \frac{A_{d_{\mathcal{U}}}}{4 \pi^{2}} g_{s}^{2}\left[\left(g_{L}^{\mathcal{U}_{V}^{\mathrm{n}} t q}\right)^{2}+\left(g_{R}^{\mathcal{U}_{V}^{\mathrm{n}} t q}\right)^{2}\right]\left(\frac{m_{t}^{2}}{\Lambda_{\mathcal{U}}^{2}}\right)^{d_{\mathcal{U}}-1} m_{t}[(a+2)-4 x]\left(1-2 x-x_{0}\right)^{d_{\mathcal{U}}-2} \frac{x^{2}}{(1-2 x)} \theta\left(1-2 x-x_{0}\right) d x$,

where $x_{0}=\mu^{2} / m_{t}^{2}$. Evaluating the integral in the limit of $\left[0,\left(1-x_{0}\right) / 2\right]$, we get the partial top decay width in the region $d_{\mathcal{U}}>1$.

[1] CDF note 9913;

[2] T. Aaltonen et al. [CDF Collaboration], Phys. Rev. D 82, 052002 (2010) arXiv:1002.2919 [hep-ex]]; T. Aal- tonen et al. [CDF Collaboration], Phys. Rev. D 83, 071102 (2011) arXiv:1007.4423 [hep-ex]]; T. Aaltonen et al. [CDF Collaboration], Phys. Rev. D 84, 031101 (2011) 
arXiv:1103.4821 [hep-ex]].

[3] V. M. Abazov et al. [D0 Collaboration], Phys. Rev. D 84, 012008 (2011) arXiv:1101.0124 [hep-ex]]; V. M. Abazov et al. [D0 Collaboration], Phys. Lett. B 704, 403 (2011) arXiv:1105.5384 [hep-ex]].

[4] N. Kidonakis, Phys. Rev. D 82, 114030 (2010) arXiv:1009.4935 [hep-ph]]. N. Kidonakis, arXiv: 1105.3481 [hep-ph].

[5] P. Baernreuther, M. Czakon and A. Mitov, arXiv:1204.5201 [hep-ph].

[6] V. M. Abazov et al. [D0 Collaboration], Phys. Rev. D 84, 112005 (2011) arXiv:1107.4995 [hep-ex]].

[7] CDF Collaboration,CDF Note 10807 http://www-cdf.fnal.gov/physics/new/top/2012/LepJe_ AFB _Winter2012/CDF10807.pdf].

[8] CDF Collaboration, CDF Note 10584 July 2011, http://www-cdf.fnal.gov/ physics/ new/top/ 2011/ AfbComb/ Afb _combo_5invfb.pdf].

[9] CDF Note 10436 http://www-cdf.fnal.gov/ physics/ new/ top/2011/ DilAfb/ cdfpubnote.pdf].

[10] T. Aaltonen et al. [CDF Collaboration], Phys. Rev. D 83, 112003 (2011) arXiv:1101.0034 [hep-ex]].

[11] O. Antunano, J. H. Kuhn and G. Rodrigo, Phys. Rev. D 77, 014003 (2008) arXiv:0709.1652 [hep-ph]]; P. Ferrario and G. Rodrigo, J. Phys. Conf. Ser. 171, 012091 (2009) arXiv:0907.0096 [hep-ph]]; S. Jung, H. Murayama, A. Pierce and J. D. Wells, Phys. Rev. D 81, 015004 (2010) arXiv:0907.4112 [hep-ph]]; Q. -H. Cao, D. McKeen, J. L. Rosner, G. Shaughnessy and C. E. M. Wagner, Phys. Rev. D 81, 114004 (2010) arXiv:1003.3461 [hep-ph]]; V. Barger, W. -Y. Keung and C. -T. Yu, Phys. Rev. D 81, 113009 (2010) arXiv:1002.1048 [hepph]]; J. Cao, Z. Heng, L. Wu and J. M. Yang, Phys. Rev. D 81, 014016 (2010) arXiv:0912.1447 [hep-ph]]; B. Xiao, Y. -k. Wang and S. -h. Zhu, Phys. Rev. D 82, 034026 (2010) arXiv:1006.2510 [hep-ph]]; A. Djouadi, G. Moreau, F. Richard and R. K. Singh, Phys. Rev. D 82, 071702 (2010) arXiv:0906.0604 [hep-ph]]. Y. k. Wang, B. Xiao and S. -h. Zhu, Phys. Rev. D 82, 094011 (2010) arXiv:1008.2685 [hep-ph]]; R. S. Chivukula, E. H. Simmons and C. -P. Yuan, Phys. Rev. D 82, 094009 (2010) arXiv:1007.0260 [hep-ph]]; D. -W. Jung, P. Ko and J. S. Lee, Phys. Lett. B 701, 248 (2011) arXiv:1011.5976 [hep-ph]]; D. Choudhury, R. M. Godbole, S. D. Rindani and P. Saha, Phys. Rev. D 84, 014023 (2011) arXiv:1012.4750 [hep-ph]]; S. Jung, A. Pierce and J. D. Wells, Phys. Rev. D 83, 114039 (2011) arXiv:1103.4835 [hep-ph]]; C. Delaunay, O. Gedalia, Y. Hochberg, G. Perez and Y. Soreq, JHEP 1108, 031 (2011) arXiv:1103.2297 [hep-ph]]; D. Krohn, T. Liu, J. Shelton and L. -T. Wang, Phys. Rev. D 84, 074034 (2011) arXiv:1105.3743 [hep-ph]]; N. Craig, C. Kilic and M. J. Strassler, Phys. Rev. D 84, 035012 (2011) arXiv:1103.2127 [hep-ph]]; R. Foot, Phys. Rev. D 83, 114013 (2011) arXiv:1103.1940 [hep-ph]]; E. R. Barreto, Y. A. Coutinho and J. Sa Borges, Phys. Rev. D 83, 054006 (2011) arXiv:1103.1266 [hep-ph]]; A. R. Zerwekh, Phys. Lett. B 704, 62 (2011) arXiv:1103.0956 [hep-ph]]; K. M. Patel and P. Sharma, JHEP 1104, 085 (2011) arXiv:1102.4736 [hep-ph]]; K. Blum, C. Delaunay, O. Gedalia, Y. Hochberg, S. J. Lee, Y. Nir, G. Perez and Y. Soreq, Phys. Lett. B 702, 364 (2011) arXiv:1102.3133 [hep-ph]]; B. Bhattacherjee, S. S. Biswal and D. Ghosh, Phys. Rev. D 83, 091501 (2011) arXiv:1102.0545 [hep-ph]]; E. L. Berger, Q. -H. Cao, C. -R. Chen, C. S. Li and H. Zhang, Phys. Rev. Lett. 106, 201801 (2011) arXiv:1101.5625 [hep-ph]]; Y. Bai, J. L. Hewett, J. Kaplan and T. G. Rizzo, JHEP 1103, 003 (2011) arXiv:1101.5203 [hep-ph]]; J. Cao, L. Wang, L. Wu and J. M. Yang, Phys. Rev. D 84, 074001 (2011) arXiv:1101.4456 [hep-ph]]; B. Xiao, Y. -K. Wang, Z. Q. Zhou and S. -h. Zhu, Phys. Rev. D 83, 057503 (2011) arXiv:1101.2507 [hep-ph]]; K. Cheung and T. -C. Yuan, Phys. Rev. D 83, 074006 (2011) arXiv:1101.1445 [hep-ph]]; G. M. Tavares and M. Schmaltz, Phys. Rev. D 84, 054008 (2011) arXiv:1107.0978 [hep-ph]]; S. Jung, A. Pierce and J. D. Wells, Phys. Rev. D 84, 091502 (2011) arXiv:1108.1802 [hep-ph]]; G. Bevilacqua, M. Czakon, C. G. Papadopoulos and M. Worek, Phys. Rev. D 84, 114017 (2011) arXiv:1108.2851 [hepph]]; M. Frank, A. Hayreter and I. Turan, Phys. Rev. D 84, 114007 (2011) arXiv:1108.0998 [hep-ph]]; J. Baglio, M. Beccaria, A. Djouadi, G. Macorini, E. Mirabella, N. Orlando, F. M. Renard and C. Verzegnassi, Phys. Lett. B 705, 212 (2011) arXiv:1109.2420 [hep-ph]]; D. Y. Shao, C. S. Li, J. Wang, J. Gao, H. Zhang and H. X. Zhu, Phys. Rev. D 84, 054016 (2011) arXiv:1107.4012 [hep-ph]]; E. Alvarez, L. Da Rold, J. I. S. Vietto and A. Szynkman, JHEP 1109, 007 (2011) arXiv:1107.1473 [hep-ph]]; L. Vecchi, JHEP 1110, 003 (2011) arXiv:1107.2933 [hep-ph]]; G. Zhu, Phys. Lett. B 703, 142 (2011) arXiv:1104.3227 [hepph]]; X. -P. Wang, Y. -K. Wang, B. Xiao, J. Xu and S. -h. Zhu, Phys. Rev. D 83, 115010 (2011) arXiv:1104.1917 [hep-ph]]; J. A. Aguilar-Saavedra and M. Perez-Victoria, Phys. Lett. B 705, 228 (2011) arXiv:1107.2120 [hep-ph]]; S. Knapen, Y. Zhao and M. J. Strassler, arXiv:1111.5857 [hep-ph]; E. Gabrielli, M. Raidal and A. Racioppi, arXiv:1112.5885 [hep-ph]; P. Ko, Y. Omura and C. Yu, arXiv:1201.1352 [hep-ph]; S. Chatrchyan et al. [CMS Collaboration], Phys. Lett. B 709, 28 (2012) arXiv:1112.5100 [hep-ex]]; L. Wang, L. Wu and J. M. Yang, Phys. Rev. D 85, 075017 (2012) arXiv:1111.4771 [hep-ph]]; E. L. Berger, Q. H. Cao, C. -R. Chen, J. -H. Yu and H. Zhang, arXiv:1111.3641 [hep-ph]; N. Kosnik, I. Dorsner, J. Drobnak, S. Fajfer and J. F. Kamenik, arXiv:1111.0477] [hepph]; K. Yan, J. Wang, D. Y. Shao and C. S. Li, Phys. Rev. D 85, 034020 (2012) arXiv:1110.6684 [hepph]]; J. N. Ng and P. T. Winslow, JHEP 1202, 140 (2012) arXiv:1110.5630 [hep-ph]]; K. Kolodziej, Phys. Lett. B 710, 671 (2012) arXiv:1110.2103 [hep-ph]]; J. H. Kuhn and G. Rodrigo, JHEP 1201, 063 (2012) arXiv:1109.6830 [hep-ph]]; J. Cao, K. Hikasa, L. Wang, L. Wu and J. M. Yang, Phys. Rev. D 85, 014025 (2012) arXiv:1109.6543 [hep-ph]]; P. Ko, Y. Omura and C. Yu, JHEP 1201, 147 (2012) arXiv:1108.4005 [hepph]]; H. Davoudiasl, T. McElmurry and A. Soni, Phys. Rev. D 85, 054001 (2012) arXiv:1108.1173 [hep-ph]]; S. S. Biswal, S. Mitra, R. Santos, P. Sharma, R. K. Singh and M. Won, arXiv:1201.3668 [hep-ph]; E. L. Berger, Q. -H. Cao, C. -R. Chen, J. -H. Yu and H. Zhang, Phys. Rev. Lett. 108, 072002 (2012) arXiv:1201.1790 [hep-ph]]; J. A. Aguilar-Saavedra, arXiv:1202.2382 [hepph]. B. Grinstein, C. W. Murphy, D. Pirtskhalava and P. Uttayarat, arXiv:1203.2183 [hep-ph]; D. Duffty, Z. Sullivan and H. Zhang, Phys. Rev. D 85, 094027 (2012) arXiv:1203.4489 [hep-ph]]; P. Ko, Y. Omura and 
C. Yu, arXiv:1205.0407 [hep-ph]; S. Y. Ayazi, S. Khatibi and M. Mohammadi Najafabadi, arXiv:1205.3311 [hepph]; K. Hagiwara and J. Nakamura, arXiv:1205.5005 [hep-ph]; B. C. Allanach and Sridhar, arXiv:1205.5170 [hep-ph]; K. Hagiwara, J. Kanzaki and Y. Takaesu, arXiv:1205.5173 [hep-ph]; D. Pagani, arXiv:1205.6182 [hep-ph]; G. Dupuis and J. M. Cline, arXiv:1206.1845 [hep-ph].

[12] D. -W. Jung, P. Ko, J. S. Lee and S. -h. Nam, Phys. Lett. B 691, 238 (2010) arXiv:0912.1105 [hep-ph]].

[13] J. Shu, K. Wang and G. Zhu, Phys. Rev. D 85, 034008 (2012) arXiv:1104.0083 [hep-ph]].

[14] M. I. Gresham, I. -W. Kim and K. M. Zurek, Phys. Rev. D 83, 114027 (2011) arXiv:1103.3501 [hep-ph]];

[15] J. A. Aguilar-Saavedra and M. Perez-Victoria, JHEP 1109, 097 (2011) arXiv:1107.0841 [hep-ph]]; J. A. Aguilar-Saavedra and M. Perez-Victoria, Phys. Rev. D 84, 115013 (2011) arXiv:1105.4606 [hep-ph]].

[16] C. -H. Chen, G. Cvetic and C. S. Kim, Phys. Lett. B 694, 393 (2011) arXiv:1009.4165 [hep-ph]].

[17] H. Georgi, Phys. Rev. Lett. 98, 221601 (2007) hep-ph/0703260.

[18] D. Choudhury and D. K. Ghosh, Int. J. Mod. Phys. A 23, 2579 (2008) arXiv:0707.2074 [hep-ph]].

[19] A. T. Alan and N. K. Pak, Europhys. Lett. 84, 11001 (2008) arXiv:0708.3802 [hep-ph]]; A. T. Alan, N. K. Pak and A. Senol, Europhys. Lett. 83, 21001 (2008) arXiv:0710.4239 [hep-ph]]; H. -F. Li, H. -l. Li, Z. G. Si and Z. -J. Yang, Commun. Theor. Phys. 51, 707 (2009) arXiv:0802.0236 [hep-ph]]; B. Sahin, Balk. Phys. Lett. 18N5, 28 (2010) I. Sahin, Eur. Phys. J. C 60, 431 (2009) arXiv:0802.2818 [hep-ph]]. T. M. Aliev, O. Cakir and K. O. Ozansoy, Phys. Lett. B 670, 336 (2009) arXiv:0809.2327 [hep-ph]]; T. M. Aliev and K. O. Ozansoy, arXiv:0905.1597 [hep-ph];

[20] M. Arai, N. Okada and K. Smolek, Phys. Rev. D 79, 074019 (2009) arXiv:0902.0418 [hep-ph]];

[21] J. H. Kuhn and G. Rodrigo, Phys. Rev. D 59, 054017 (1999) hep-ph/9807420. J. H. Kuhn and G. Rodrigo, Phys. Rev. Lett. 81, 49 (1998) hep-ph/9802268. M. T. Bowen, S. D. Ellis and D. Rainwater, Phys. Rev. D 73, 014008 (2006) hep-ph/0509267. L. G. Almeida, G. F. Sterman and W. Vogelsang, Phys. Rev. D 78, 014008 (2008) arXiv:0805.1885 [hep-ph]]; V. Ahrens, A. Ferroglia, M. Neubert, B. D. Pecjak and L. L. Yang, Phys. Rev. D 84, 074004 (2011) arXiv:1106.6051 [hepph]]; W. Bernreuther and Z. -G. Si, arXiv:1205.6580 [hep$\mathrm{ph}]$.

[22] N. Kidonakis, Phys. Rev. D 84, 011504 (2011) arXiv:1105.5167 [hep-ph]].

[23] W. Beenakker, A. Denner, W. Hollik, R. Mertig, T. Sack and D. Wackeroth, Nucl. Phys. B 411, 343 (1994); C. Kao, G. A. Ladinsky and C. P. Yuan, Int. J. Mod. Phys. A 12, 1341 (1997).

[24] A. Brandenburg, Phys. Lett. B 388, 626 (1996) hep-ph/9603333; A. Brandenburg, Eur. Phys. J. C 33, S469 (2004) hep-ph/0310268. K. Melnikov and M. Schulze, Phys. Lett. B 700, 17 (2011) arXiv:1103.2122 [hep-ph]]. S. J. Parke, arXiv:1202.2345 [hep-ph].

[25] V. M. Abazov et al. [D0 Collaboration], Phys. Rev. Lett. 108, 032004 (2012) arXiv:1110.4194 [hep-ex]]. G. Aad et al. [ATLAS Collaboration], Phys. Rev. Lett. 108, 212001 (2012) arXiv:1203.4081 [hep-ex]].
[26] G. Mahlon and S. J. Parke, Phys. Lett. B 411, 173 (1997) hep-ph/9706304; G. Mahlon and S. J. Parke, Phys. Rev. D 53, 4886 (1996) hep-ph/9512264.

[27] T. Stelzer and S. Willenbrock, Phys. Lett. B 374, 169 (1996) hep-ph/9512292.

[28] T. Aaltonen et al. [CDF Collaboration], Phys. Rev. D 83, 031104 (2011) arXiv:1012.3093 [hep-ex]].

[29] CDF note 10719-CONF http://www-cdf.fnal.gov/ physics/new/top/2011/] V. M. Abazov et al. [D0 Collaboration], Phys. Lett. B 702, 16 (2011) arXiv:1103.1871 [hep-ex]];

[30] K. Bloom [D0 Collaboration], arXiv:1109.3691 [hep-ex].

[31] W. Bernreuther and Z. -G. Si, Nucl. Phys. B 837, 90 (2010) arXiv:1003.3926 [hep-ph]];

[32] C. Kao, Phys. Lett. B 348, 155 (1995) hep-ph/9411337. C. -S. Li, R. J. Oakes, J. M. Yang and C. P. Yuan, Phys. Lett. B 398, 298 (1997) hep-ph/9701350. S. Gopalakrishna, T. Han, I. Lewis, Z. -g. Si and Y. -F. Zhou, Phys. Rev. D 82, 115020 (2010) arXiv:1008.3508 [hepph]]. R. M. Godbole, K. Rao, S. D. Rindani and R. K. Singh, JHEP 1011, 144 (2010) arXiv:1010.1458 [hep-ph]]. C. Kao and D. Wackeroth, Phys. Rev. D 61, 055009 (2000) hep-ph/9902202. K. -i. Hikasa, J. M. Yang and B. -L. Young, Phys. Rev. D 60, 114041 (1999) hep-ph/9908231; P. -Y. Li, G. -R. Lu, J. M. Yang and H. Zhang, Eur. Phys. J. C 51, 163 (2007) hep-ph/0608223; J. Cao, L. Wu and J. M. Yang, Phys. Rev. D 83, 034024 (2011) arXiv:1011.5564 [hep-ph]].

[33] D. Chang, S. -C. Lee and A. Sumarokov, Phys. Rev. Lett. 77, 1218 (1996) hep-ph/9512417]; S. Fajfer, J. F. Kamenik and B. Melic, arXiv:1205.0264 [hep-ph].

[34] T. Banks and A. Zaks, Nucl. Phys. B 196, 189 (1982). [35]

[35] H. Davoudiasl, Phys. Rev. Lett. 99, 141301 (2007) arXiv:0705.3636 [hep-ph]]; A. Freitas and D. Wyler, JHEP 0712, 033 (2007) arXiv:0708.4339 [hep-ph]].

[36] V. Barger, Y. Gao, W. -Y. Keung, D. Marfatia and V. N. Senoguz, Phys. Lett. B 661, 276 (2008) arXiv:0801.3771 [hep-ph]].

[37] H. Georgi, Phys. Lett. B 650, 275 (2007) arXiv:0704.2457 [hep-ph]].

[38] T. M. Aliev, A. S. Cornell and N. Gaur, JHEP 0707, 072 (2007) arXiv:0705.4542 [hep-ph]]; T. M. Aliev, A. S. Cornell and N. Gaur, Phys. Lett. B 657, 77 (2007) arXiv:0705.1326 [hep-ph]]; K. Cheung, W. Y. Keung and T. -C. Yuan, Phys. Rev. D 76, 055003 (2007) arXiv:0706.3155 [hep-ph]]; Y. Liao and J. -Y. Liu, Phys. Rev. Lett. 99, 191804 (2007) arXiv:0706.1284 [hep-ph]]; Y. Liao, Phys. Rev. D 76, 056006 (2007) arXiv:0705.0837 [hep-ph]]; T. G. Rizzo, JHEP 0710, 044 (2007) arXiv:0706.3025 [hep-ph]]; K. Cheung, W. Y. Keung and T. -C. Yuan, Phys. Rev. Lett. 99, 051803 (2007) arXiv:0704.2588 [hep-ph]]; T. Kikuchi, N. Okada and M. Takeuchi, Phys. Rev. D 77, 094012 (2008) arXiv:0801.0018 [hep-ph]]; T. Kikuchi and N. Okada, Phys. Lett. B 661, 360 (2008) arXiv:0707.0893 [hep-ph]]; C. H. Chen and C. Q. Geng, Can. J. Phys. 86 (2008) 641; J. Bergstrom and T. Ohlsson, Phys. Rev. D 80 (2009) 115014 [arXiv:0909.2213 [hep-ph]]. A. Moyotl, A. Rosado and G. Tavares-Velasco, Phys. Rev. D 84 (2011) 073010 arXiv:1109.4890 [hep-ph]]; [CMS Collaboration], CMSPAS-EXO-09-011; G. A. Kozlov, arXiv:1002.2859 [hepph]; L. -G. Bian, arXiv:1108.1538 [hep-ph]; E. O. Iltan, Int. J. Mod. Phys. A 27 (2012) 1250055 arXiv:1112.0960 
[hep-ph]].

[39] I. Lewis, arXiv:0710.4147 [hep-ph]; P. K. Das, Phys. Rev. D 76, 123012 (2007) arXiv:0708.2812 [hep-ph]]; A. Freitas and D. Wyler, JHEP 0712, 033 (2007) arXiv:0708.4339 [hep-ph]]; S. Hannestad, G. Raffelt and Y. Y. Y. Wong, Phys. Rev. D 76, 121701 (2007) arXiv:0708.1404 [hep-ph]]; T. Kikuchi and N. Okada, Phys. Lett. B 665, 186 (2008) arXiv:0711.1506 [hepph]]; G. L. Alberghi, A. Y. Kamenshchik, A. Tronconi, G. P. Vacca and G. Venturi, Phys. Lett. B 662, 66 (2008) arXiv:0710.4275 [hep-th]]; H. Goldberg and P. Nath, Phys. Rev. Lett. 100, 031803 (2008) arXiv:0706.3898 [hep-ph]]; J. R. Mureika, Phys. Lett. B 660, 561 (2008) arXiv:0712.1786 [hep-ph]]; S. Dutta and A. Goyal, Phys. Lett. B 664, 25 (2008) arXiv:0801.2143 [hep-ph]]; S. L. Chen, X. -G. He, X. -P. Hu and Y. Liao, Eur. Phys. J. C 60, 317 (2009) arXiv:0710.5129 [hep-ph]]; J. McDonald, JCAP 0903, 019 (2009) arXiv:0709.2350 [hep-ph]]; R. A. de Souza and J. E. Horvath, arXiv:1206.2784 [grqc].

[40] M. Bander, J. L. Feng, A. Rajaraman and Y. Shirman, Phys. Rev. D 76, 115002 (2007) arXiv:0706.2677 [hep-ph]]; M. A. Stephanov, Phys. Rev. D 76, 035008 (2007) arXiv:0705.3049 [hep-ph]]; M. J. Strassler, arXiv:0801.0629 [hep-ph]; N. G. Deshpande, S. D. H. Hsu and J. Jiang, Phys. Lett. B 659, 888 (2008) arXiv:0708.2735 [hep-ph]]; Y. Liao, Eur. Phys. J. C 55, 483 (2008) arXiv:0708.3327 [hep-ph]]; M. PerezVictoria, JHEP 0901, 011 (2009) arXiv:0808.4075 [hepph]]. A. Rajaraman, AIP Conf. Proc. 1078, 63 (2009) arXiv:0809.5092 [hep-ph]];

[41] B. Grinstein, K. A. Intriligator and I. Z. Rothstein, Phys. Lett. B 662, 367 (2008) arXiv:0801.1140 [hep-ph]]. G. Mack and K. Symanzik, Commun. Math. Phys. 27, 247 (1972).

[42] J. -P. Lee, arXiv:0911.5382 [hep-th].

[43] G. Cacciapaglia, G. Marandella and J. Terning, JHEP 0801, 070 (2008) arXiv:0708.0005 [hep-ph]].

[44] P. Gaete and E. Spallucci, Phys. Lett. B 661, 319 (2008) arXiv:0801.2294 [hep-th]]; J. Galloway, D. Martin and D. Stancato, arXiv:0802.0313 [hep-th]. A. L. Licht and W. -Y. Keung, arXiv:0806.3596 [hep-th]; Y. Liao, Eur. Phys. J. C 60, 125 (2009) arXiv:0804.4033 [hep-ph]]; R. Basu, D. Choudhury and H. S. Mani, Eur. Phys. J. C 61, 461 (2009) arXiv:0803.4110 [hep-ph]]. A. Ilderton, Phys. Rev. D 79, 025014 (2009) arXiv:0810.3916 [hep-th]]; G. A. Kozlov, Phys. Part. Nucl. 41 (2010) 957 arXiv:0905.2272 [hep-ph]].

[45] M. Bahr, S. Gieseke, M. A. Gigg, D. Grellscheid,
K. Hamilton, O. Latunde-Dada, S. Platzer and P. Richardson et al., Eur. Phys. J. C 58, 639 (2008) arXiv:0803.0883 [hep-ph]].

[46] A. Belyaev, N. D. Christensen and A. Pukhov, arXiv:1207.6082 [hep-ph].

[47] J. Alwall, P. Demin, S. de Visscher, R. Frederix, M. Herquet, F. Maltoni, T. Plehn and D. L. Rainwater et al., JHEP 0709, 028 (2007) arXiv:0706.2334 [hep-ph]]. J. Alwall, M. Herquet, F. Maltoni, O. Mattelaer and T. Stelzer, JHEP 1106, 128 (2011) arXiv:1106.0522 [hep-ph]].

[48] T. Aaltonen et al. [CDF Collaboration], Phys. Rev. D 79, 112002 (2009) [arXiv:0812.4036 [hep-ex]].

[49] N. Agarwal, M. C. Kumar, P. Mathews, V. Ravindran and A. Tripathi, Phys. Rev. D 80, 035015 (2009) arXiv:0903.0202 [hep-ph]].

[50] D. Choudhury, D. K. Ghosh and Mamta, Phys. Lett. B 658, 148 (2008) arXiv:0705.3637 [hep-ph]].

[51] J. A. Aguilar-Saavedra and M. Perez-Victoria, Phys. Lett. B 701, 93 (2011) arXiv:1104.1385 [hep-ph]].

[52] S. Chatrchyan et al. [CMS Collaboration], JHEP 1108, 005 (2011) arXiv:1106.2142 [hep-ex]].

[53] The CDF Collaboration (2011), CDF/PHYS/EXO/ PUBLIC/ 10466; [URL http://www-cdf.fnal.gov].

[54] V. M. Abazov et al. [D0 Collaboration], Phys. Rev. Lett. 106, 022001 (2011) arXiv:1009.5686 [hep-ex]].

[55] T. Aaltonen et al. [CDF Collaboration], Phys. Rev. Lett. 101, 192002 (2008) arXiv:0805.2109 [hep-ex]].

[56] V. M. Abazov et al. [D0 Collaboration], Phys. Lett. B 701, 313 (2011) arXiv:1103.4574 [hep-ex]].

[57] R. Mohanta and A. K. Giri, Phys. Lett. B 660, 376 (2008) arXiv:0711.3516 [hep-ph]].

[58] J. K. Parry, Phys. Rev. D 78, 114023 (2008) arXiv:0806.4350 [hep-ph]].

[59] G. Aad et al. [ATLAS Collaboration], JHEP 1209, 041 (2012) arXiv:1207.2409 [hep-ex]].

[60] S. Chatrchyan et al. [CMS Collaboration], arXiv:1209.4397 [hep-ex]].

[61] CMS PASS EXO-10-001 http://cms-physics.web.cern ch/cms-physics/public/EXO-10-001-pas.pdf]

[62] CMS PASS QCD-10-011 http://cdsweb.cern.ch/record/ 1280682/files/QCD-10-011-pas.pdf]

[63] ATLAS-CONF-2012-134 http://cdsweb.cern.ch/record/ 1478422/files/ATLAS-CONF-2012-134.pdf]

[64] G. Aad et al. [ATLAS Collaboration], arXiv:1206.0257 [hep-ex].

[65] S. Chatrchyan et al. [CMS Collaboration], arXiv:1208.0957 [hep-ex]. 